\title{
Double Quasi-Poisson Brackets: Fusion and New Examples
}

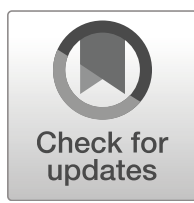

\section{Maxime Fairon ${ }^{1}$}

Received: 18 July 2019 / Accepted: 8 June 2020 / Published online: 30 June 2020

(C) The Author(s) 2020

\begin{abstract}
We exhibit new examples of double quasi-Poisson brackets, based on some classification results and the method of fusion. This method was introduced by Van den Bergh for a large class of double quasi-Poisson brackets which are said differential, and our main result is that it can be extended to arbitrary double quasi-Poisson brackets. We also provide an alternative construction for the double quasi-Poisson brackets of Van den Bergh associated to quivers, and of Massuyeau-Turaev associated to the fundamental groups of surfaces.
\end{abstract}

Keywords Double bracket · Quasi-Hamiltonian algebra · Non-commutative geometry

Mathematics Subject Classification (2010) 16 S38 $\cdot 17 \mathrm{~B} 63 \cdot 53 \mathrm{D} 17$

\section{Introduction}

We fix a finitely generated associative unital algebra $A$ over a field $\mathbb{k}$ of characteristic 0 , and we write $\otimes=\bigotimes_{\mathbb{k}}$ for brevity. Following Van den Bergh's initial construction [20], we define on $A$ a double bracket $\{\{-,-\}\}: A \times A \rightarrow A \otimes A$ as a $\mathbb{k}$-bilinear map satisfying for any $a, b, c \in A$

$$
\{\{a, b\}\}=-\{\{b, a\}\}^{\circ} \quad \text { (cyclic antisymmetry), }
$$

where $(-)^{\circ}$ denotes the permutation of factors in $A \otimes A$, together with

$$
\{\{a, b c\}\}=\{\{a, b\}\} c+b\{\{a, c\}\} \quad \text { (right derivation rule). }
$$

Here, the multiplication refers to the outer $A$-bimodule structure on $A \otimes A$, that is $a d b=$ $\left(a d^{\prime}\right) \otimes\left(d^{\prime \prime} b\right)$ under Sweedler's notation $d=d^{\prime} \otimes d^{\prime \prime} \in A \otimes A$, which we use throughout this text. Assuming that Eq. 1.1 holds, one can easily check that Eq. 1.2 is equivalent to

$$
\{\{b c, a\}\}=\{\{b, a\}\} * c+b *\{\{c, a\}\} \quad \text { (left derivation rule), }
$$

Presented by: Iain Gordon

Maxime Fairon

Maxime.Fairon@glasgow.ac.uk

1 School of Mathematics and Statistics, University of Glasgow, University Place, Glasgow, G12 8QQ, UK 
where this time $*$ denotes the inner $A$-bimodule structure on $A \otimes A$ given by $a *\left(d^{\prime} \otimes d^{\prime \prime}\right) * b=$ $\left(d^{\prime} b\right) \otimes\left(a d^{\prime \prime}\right)$. From these derivation rules, it is easily seen that it suffices to define double brackets on generators of $A$. Associated to such a double bracket, we can define an operation $A^{\times 3} \rightarrow A^{\otimes 3}$ by setting

$$
\{\{a, b, c\}\}=\left\{\left\{a,\{\{b, c\}\}^{\prime}\right\}\right\} \otimes\{\{b, c\}\}^{\prime \prime}+\tau_{(123)}\left\{\left\{b,\{\{c, a\}\}^{\prime}\right\}\right\} \otimes\{\{c, a\}\}^{\prime \prime}+\tau_{(123)}^{2}\left\{\left\{c,\{\{a, b\}\}^{\prime}\right\}\right\} \otimes\{\{a, b\}\}^{\prime \prime} .
$$

(Here, we define $\tau_{(123)}: A^{\otimes 3} \rightarrow A^{\otimes 3}$ by $\tau_{(123)}\left(a_{1} \otimes a_{2} \otimes a_{3}\right)=a_{3} \otimes a_{1} \otimes a_{2}$.) This map is an instance of triple bracket : a $\mathbb{k}$-trilinear map, which is also a derivation in its last argument for the outer bimodule structure of $A^{\otimes 3}$, and which satisfies a generalisation of the cyclic antisymmetry Eq. 1.1 :

$$
\tau_{(123)} \circ\{\{-,-,-\}\} \circ \tau_{(123)}^{-1}=\{\{-,-,-\}\} .
$$

An important class of double brackets consists of double Poisson brackets. They are such that the associated triple brackets $\{\{-,-,-\}\}$ identically vanish. Using Eq. 1.4, this condition can be seen as a version of Jacobi identity with value in $A^{\otimes 3}$. These structures have also been introduced by Van den Bergh [20], and have been a recent subject of study, see e.g. [4, 10, 15-19, 22].

Another interesting class of double brackets appears when the unit in $A$ admits a decomposition $1=\sum_{s \in I} e_{s}$ in terms of a finite set of orthogonal idempotents, i.e. $|I| \in \mathbb{N}^{\times}$and $e_{s} e_{t}=\delta_{s t} e_{s}$. In that case, we view $A$ as a $B$-algebra for $B=\oplus_{s \in I} \mathbb{k} e_{s}$, and we naturally extend the definition of a double bracket to require $B$-bilinearity, i.e. it vanishes when one of the arguments belongs to $B$. Then, we say that the double bracket is quasi-Poisson, or that $(A,\{\{-,-\}\})$ is a double quasi-Poisson algebra, if the associated triple bracket (1.4) satisfies the relation

$$
\begin{aligned}
\{\{a, b, c\}\}= & \frac{1}{4} \sum_{s \in I}\left(c e_{s} a \otimes e_{s} b \otimes e_{s}-c e_{s} a \otimes e_{s} \otimes b e_{s}-c e_{s} \otimes a e_{s} b \otimes e_{s}+c e_{s} \otimes a e_{s} \otimes b e_{s}\right. \\
& \left.-e_{s} a \otimes e_{s} b \otimes e_{s} c+e_{s} a \otimes e_{s} \otimes b e_{s} c+e_{s} \otimes a e_{s} b \otimes e_{s} c-e_{s} \otimes a e_{s} \otimes b e_{s} c\right)(1.6)
\end{aligned}
$$

on any $a, b, c \in A$. Condition Eq. 1.6 is an expanded form of the original definition [20, $\S 5.1]$, and only needs to be checked on generators by the properties of a triple bracket. The main interest of this form is that it is easier to handle in order to classify double quasiPoisson brackets. Indeed, up to now few cases of double quasi-Poisson brackets are known except associated to quivers [20,21] or fundamental groups of surfaces [14]. To have more examples, we provide a complete classification on the free algebra over one generator, and continue the investigation for two generators (with some restrictions).

The reader could then be tempted to say that such examples do not provide particular insights about double quasi-Poisson brackets in general. However, an important result of Van den Bergh is that we can perform fusion [20, §5.3] : we can identify idempotents in an algebra with a double quasi-Poisson bracket, and the resulting algebra also admits a double quasi-Poisson bracket. For example, if we respectively denote by $e_{1}, e_{2}$ the units of $\mathbb{k}[t], \mathbb{k}\left\langle s_{1}, s_{2}\right\rangle$ viewed as orthogonal idempotents inside $\mathbb{k}[t] \oplus \mathbb{k}\left\langle s_{1}, s_{2}\right\rangle$, the fusion algebra obtained by the identification of $e_{1}$ and $e_{2}$ is nothing else than $\mathbb{k}\left\langle t, s_{1}, s_{2}\right\rangle$. Hence, knowing a double quasi-Poisson bracket before fusion gives another one on the free algebra over three generators. Therefore, our classification allows to get double quasi-Poisson brackets over any free algebra in general, though not all of them. Moving to more exotic examples of double quasi-Poisson algebras, there was a major obstruction to use this fusion process up to now, as we needed the double quasi-Poisson bracket to be differential, see $\S 2.1$ for 
the definition. It was expected by Van den Bergh that this assumption could be removed $[20, \S 5.3]$, and the main aim of this paper is to prove this result in its most general form.

Theorem 1.1 (cf. Theorem 2.14) Let $(A,\{\{-,-\}\})$ be a double quasi-Poisson B-algebra, with $B=\oplus_{s \in I} \mathbb{k} e_{s},|I| \in \mathbb{N}^{\times}$, where $e_{s} e_{t}=\delta_{s t} e_{s}$ for any $s, t \in I$. Then, if we pick $s, t \in I$ distinct, the algebra $A^{\prime}$ obtained by identifying the idempotents $e_{s}, e_{t} \in A$ has a double quasi-Poisson bracket which coincides with the image of $\{\{-,-\}\}$ on $\oplus_{s^{\prime}, t^{\prime} \in I^{\prime}} e_{s^{\prime}} A^{\prime} e_{t^{\prime}}$, where $I^{\prime}=I \backslash\{1,2\}$.

The advantage of our proof of this theorem is to get an explicit form for the double quasiPoisson bracket in the algebra $A^{\prime}$ obtained by identification of the idempotents $e_{s}, e_{t} \in A$ : it is given in terms of the double bracket on $A$, together with a second double bracket computed in Lemma 2.19 which was uncovered by Van den Bergh [20, Theorem 5.3.1]. Therefore, it becomes easy to see when a double quasi-Poisson bracket has been obtained by fusion. In particular, we can show using our classification of double quasi-Poisson bracket on the free algebra on two generators (with some mild restrictions) provided in $\S 4.3$ that any such double bracket is isomorphic to one obtained by fusion, see Theorem 4.10. This unexpected result suggests that knowing double quasi-Poisson brackets on $\mathbb{k}[t]$ and the path algebra of the (double of the) one-arrow quiver $t: 1 \rightarrow 2$ may be enough to obtain most examples of double quasi-Poisson algebra structures on free algebras.

A particular subclass of double quasi-Poisson brackets consists in those that admit a distinguished element. To be precise, given a double quasi-Poisson algebra $(A,\{\{-,-\}\})$ as above with a complete set of orthogonal idempotents $\left(e_{s}\right)_{s \in I}$, a multiplicative moment map is an invertible element $\Phi=\sum_{s \in I} \Phi_{s}$ with $\Phi_{s} \in e_{s} A e_{s}$ such that we have for all $a \in A$ and $s \in I$

$$
\left\{\left\{\Phi_{s}, a\right\}\right\}=\frac{1}{2}\left(a e_{s} \otimes \Phi_{s}-e_{s} \otimes \Phi_{s} a+a \Phi_{s} \otimes e_{s}-\Phi_{s} \otimes e_{s} a\right) .
$$

We say that the triple $(A,\{\{-,-\}\}, \Phi)$ is a quasi-Hamiltonian algebra. As a continuation of the previous result, Van den Bergh showed that we can also obtain a moment map after fusion inside a quasi-Hamiltonian algebra when the double bracket is differential [20, Theorem 5.3.2]. We also show that this result can be extended to the general case, see Theorem 2.15. As a by-product of our method to prove that we keep a double quasi-Poisson bracket or multiplicative moment map after fusion, we can easily recover the double quasi-Poisson brackets of Van den Bergh [20] and Massuyeau-Turaev [14], see Theorems 3.3 and 3.5.

To finish this introduction, let us recall that double brackets have been introduced by Van den Bergh as a non-commutative version of an antisymmetric biderivation following the non-commutative principle formulated by Kontsevich and Rosenberg [11, 12]. More precisely, as explained in $\$ 5.1$, any double bracket on an algebra $A$ gives rise to an antisymmetric biderivation on the algebra $\mathbb{k}[\operatorname{Rep}(A, n)]$ for any $n \geq 1$, i.e. on the coordinate ring of the representation space $\operatorname{Rep}(A, n)$ parametrising $n$-dimensional representations of $A$. In the same way, a double (quasi-)Poisson bracket provides a non-commutative notion of a (quasi)Poisson bracket under this non-commutative principle. Hence, the present study can be understood as giving new examples of quasi-Poisson brackets on representation spaces.

This article proceeds as follows. In Section 2, we recall the necessary constructions needed to understand the fusion procedure, and then prove the main result of this paper which is the fusion of quasi-Hamiltonian algebras in the general case. In light of those developments, we give in Section 3 some examples of double quasi-Poisson brackets obtained by fusion. We also give an alternative (though equivalent) construction of Van den Bergh's quasi-Hamiltonian algebras associated to quivers, and those of Massuyeau-Turaev associated to the fundamental group of compact surfaces with boundary. In Section 4, we get 
some first classification results for double quasi-Poisson brackets. We finish by explaining in Section 5 the notion of quasi-Poisson algebra, which is the structure carried by the coordinate ring of representation spaces of double quasi-Poisson algebras. There are four appendices that contain some computational proofs.

\section{Fusion of quasi-Hamiltonian algebras}

We consider finitely generated algebras $A, B$ over a field $\mathbb{k}$ of characteristic zero. We assume that $A$ is a $B$-algebra and, without loss of generality, we identify $B$ with its image in $A$. Our goal is to prove the main theorems of this paper, which are presented in \$2.2. To state and prove these results, we need some preliminary constructions associated to double brackets, which were already introduced by Van den Bergh in [20] for most of them. Since these results easily extend to the case of $n$-brackets (see below for the definition, noting that double brackets are 2-brackets), we begin by introducing the objects that we will use in full generalities.

\subsection{Preliminary results}

We equip the algebra $A^{\otimes n}$ with the outer $A$-bimodule structure which is given by $b\left(a_{1} \otimes\right.$ $\left.\ldots \otimes a_{n}\right) c=b a_{1} \otimes \ldots \otimes a_{n} c$. For any $s \in S_{n}$, we introduce the map $\tau_{s}: A^{\otimes n} \rightarrow A^{\otimes n}$ defined by $\tau_{s}\left(a_{1} \otimes \ldots \otimes a_{n}\right)=a_{s^{-1}(1)} \otimes \ldots \otimes a_{s^{-1}(n)}$. Following Van den Bergh [20], we say that a $B$ linear map $\{\{-, \ldots,-\}\}: A^{\times n} \rightarrow A^{\otimes n}$ is a $n$-bracket if it is a derivation in its last argument for the outer bimodule structure on $A^{\otimes n}$, and if it is cyclically anti-symmetric :

$$
\tau_{(1 \ldots n)} \circ\{\{-, \ldots,-\}\} \circ \tau_{(1 \ldots n)}^{-1}=(-1)^{n+1}\{\{-, \ldots,-\}\} .
$$

By $B$-linearity, we mean that the map $\{\{-, \ldots,-\}\}$ is $\mathbb{k}$-linear in each argument and it vanishes on any subset $A^{\times i-1} \times B \times A^{n-i}, 1 \leq i \leq n$. Double and triple brackets as defined in the introduction can be equivalently obtained from the above formulation, for which they correspond to the cases $n=2$ and $n=3$.

\subsubsection{Poly-vector fields and $n$-brackets}

Examples of $n$-brackets can easily be obtained by choosing $n$ double derivations, which are elements of $\operatorname{Der}_{B}(A, A \otimes A)$, with $A \otimes A$ equipped with the outer bimodule structure. To state the result, we set $D_{A / B}:=\operatorname{Der}_{B}(A, A \otimes A)$ and we see $D_{A / B}$ as an $A$-bimodule by using the inner bimodule structure on $A \otimes A$ : if $\delta \in D_{A / B}$ and $a, b, c \in A$, then $(b \delta c)(a)=$ $\delta(a)^{\prime} c \otimes b \delta(a)^{\prime \prime}$. We then form the tensor algebra $D_{B} A:=T_{A} D_{A / B}$ of this bimodule, which is a graded algebra if we put $A$ in degree 0 and $D_{A / B}$ in degree 1 . Its elements are called poly-vector fields.

Proposition 2.1 ([20, Proposition 4.1.1])

There is a well-defined linear map $\mu:\left(D_{B} A\right)_{n} \rightarrow\{B$-linear n-brackets on $A\}, Q \mapsto$ $\{\{-, \ldots,-\}\}_{Q}$, which on $Q=\delta_{1} \ldots \delta_{n}$ is given by

$$
\begin{gathered}
\{\{-, \ldots,-\}\}_{Q}=\sum_{i=0}^{n-1}(-1)^{(n-1) i} \tau_{(1 \ldots n)}^{i} \circ\left\{\{-, \ldots,-\} \tilde{\}}_{Q} \circ \tau_{(1 \ldots n)}^{-i},\right. \\
\left\{\left\{a_{1}, \ldots, a_{n}\right\}\right\}_{Q}=\delta_{n}\left(a_{n}\right)^{\prime} \delta_{1}\left(a_{1}\right)^{\prime \prime} \otimes \delta_{1}\left(a_{1}\right)^{\prime} \delta_{2}\left(a_{2}\right)^{\prime \prime} \otimes \ldots \otimes \delta_{n-1}\left(a_{n-1}\right)^{\prime} \delta_{n}\left(a_{n}\right)^{\prime \prime} .
\end{gathered}
$$

The map $\mu$ factors through $D_{B} A /\left[D_{B} A, D_{B} A\right]$, where $[-,-]$ is the graded commutator. 
We say that a $n$-bracket is differential if it is given by $\mu(Q)$ for some $Q \in\left(D_{B} A\right)_{n}$. For example, given some $\delta_{1} \delta_{2} \in\left(D_{B} A\right)_{2}$ we have a differential double bracket by setting

$$
\{\{b, c\}\}_{\delta_{1} \delta_{2}}=\delta_{2}(c)^{\prime} \delta_{1}(b)^{\prime \prime} \otimes \delta_{1}(b)^{\prime} \delta_{2}(c)^{\prime \prime}-\delta_{1}(c)^{\prime} \delta_{2}(b)^{\prime \prime} \otimes \delta_{2}(b)^{\prime} \delta_{1}(c)^{\prime \prime},
$$

for any $b, c \in A$. Any differential double bracket is a linear sum of such double brackets.

By [5], we can write $D_{A / B}=\operatorname{Hom}_{A \otimes A^{o p}}\left(\Omega_{B}^{1} A, A \otimes A\right)$, where $\Omega_{B}^{1} A$ is the $A$-bimodule of non-commutative 1-forms relative to $B$ [9]. The bimodule $\Omega_{B}^{1} A$ allows us to give conditions for the map $\mu$ to be an isomorphism.

Proposition 2.2 ([20, Proposition 4.1.2]) Assume that $A$ is left and right flat over $B$, and that $\Omega_{B}^{1} A$ is a projective A-bimodule. Then the map $\mu$ from Proposition 2.1 is an isomorphism.

Example 2.3 Consider $\mathbb{k}[x]$, with double bracket given by $\{\{x, x\}\}=\frac{1}{2}\left(x^{2} \otimes 1-1 \otimes x^{2}\right)$ (it is quasi-Poisson by Proposition 4.1). This double bracket is differential : for $d_{x} \in D_{\mathbb{k}[x] / \mathbb{k}}$ given by $d_{x}(x)=1 \otimes 1$, we have that $P=\frac{1}{2} x^{2} d_{x} d_{x} \in\left(D_{\mathbb{k}} \mathbb{k}[x]\right)_{2}$ defines $\{\{-,-\}\}$ using Proposition 2.1.

Fix $k \geq 3$. It is not hard to see that $\left\{\left\{x, x^{k}\right\}\right\} \in I_{k} \otimes \mathbb{k}[x]+\mathbb{k}[x] \otimes I_{k}$ for $I_{k}$ the ideal generated by $x^{k}$, so that the double bracket factors as a map $A_{k} \times A_{k} \rightarrow A_{k} \otimes A_{k}$ with $A_{k}=\mathbb{k}[x] / I_{k}$. We claim that the double bracket is no longer differential on $A_{k}$. Indeed, any element $P \in D_{A_{k} / \mathbb{k}}$ is uniquely defined by the image of the generator $x$, so it can be decomposed as

$$
P(x)=c_{0,0} 1 \otimes 1+c_{1,0} x \otimes 1+c_{1,1} 1 \otimes x+\sum_{a=2}^{2 k-1} \sum_{b=0}^{a} c_{a, b} x^{b} \otimes x^{a-b}, \quad c_{a, b} \in \mathbb{k},
$$

and since we need to satisfy $P\left(x^{k}\right)=0$, we obtain that

$$
P(x)=c(x \otimes 1-1 \otimes x)+\sum_{a=2}^{2 k-1} \sum_{b=0}^{a} c_{a, b} x^{b} \otimes x^{a-b}, \quad c, c_{a, b} \in \mathbb{k},
$$

with possible relations between the coefficients $\left(c_{a, b}\right)$. If we consider arbitrary $P, Q \in D_{A_{k} / \mathbb{k}}$ of that form, we see that the double bracket they define by Eq. $2.1 \mathrm{~b}$ can be written as

$$
\{\{x, x\}\}_{P Q}=Q(x)^{\prime} P(x)^{\prime \prime} \otimes P(x)^{\prime} Q(x)^{\prime \prime}-P(x)^{\prime} Q(x)^{\prime \prime} \otimes Q(x)^{\prime} P(x)^{\prime \prime}=\sum_{a \geq 3} \sum_{b=0}^{a} d_{a, b} x^{b} \otimes x^{a-b},
$$

for some $d_{a, b} \in \mathbb{k}$. Thus, any differential double bracket $\{\{-,-\}\}$ on $A_{k}$ is such that $\{\{x, x\}\} \in$ $A_{k} \otimes A_{k}$ has homogeneous components of degree $\geq 3$, where we define the degree of $x^{a} \otimes x^{b}$ as $a+b$. Hence, the double bracket on $A_{k}$ given by $\{\{x, x\}\}=\frac{1}{2}\left(x^{2} \otimes 1-1 \otimes x^{2}\right)$ can not be differential.

The algebra $D_{B} A$ is a noncommutative version of the algebra of polyvector fields on a manifold : $D_{B} A$ admits a canonical double Schouten-Nijenhuis bracket, which makes $D_{B} A$ into a double Gerstenhaber algebra [20, \$2.7,3.2]. We write this (graded) double bracket $\left(D_{B} A\right)^{\times 2} \rightarrow\left(D_{B} A\right)^{\otimes 2}$ as $\{\{-,-\}\}_{S N}$. We denote by $\{-,-\}_{\text {SN }}$ the associated bracket $\{-,-\}_{\mathrm{SN}}:=m \circ\{\{-,-\}\}_{\mathrm{SN}}$, where $m$ is the multiplication on the algebra $D_{B} A$. We note that the following results hold.

Proposition 2.4 ([20, §4.2]) Assume that $\{\{-,-\}\}$ is a double bracket defined by the bivector $P \in\left(D_{B} A\right)_{2}$. Then the associated triple bracket given by Eq. 1.4 is defined by the trivector $\frac{1}{2}\{P, P\}_{\mathrm{SN}} \in\left(D_{B} A\right)_{3}$.

Proposition 2.5 ([20, §3.4]) Assume $e \in B$ is an idempotent such that $B e B=B$. Then $e\left(D_{B} A\right) e=D_{e B e} e A e$, and the (graded) double bracket $\{\{-,-\}\}_{\mathrm{SN}}$ on $D_{B} A$ restricted to $e\left(D_{B} A\right) e$ coincides with the double Schouten-Nijenhuis bracket on $D_{e B e} e A e$. 


\subsubsection{Induced brackets and fusion algebras}

We now state several ways to get new $n$-brackets from old ones. Most of these results are straightforward extensions of propositions given in [20, §2.5], which were originally stated in the case $n=2$.

Given an algebra $A$ over $B$ and a non-empty subset $S \subset A$, we can consider the universal localisation $A_{S}$ as an algebra over $B$. The morphism $f: A \rightarrow A_{S}$ induces a unique map of double derivations $f_{*}: D_{A / B} \rightarrow D_{A_{S} / B}$ which satisfies $f_{*}(\delta)\left(s^{-1}\right)=s^{-1} f\left(\delta(s)^{\prime}\right) \otimes$ $f\left(\delta(s)^{\prime \prime}\right) s^{-1}$ for any $\delta \in D_{B} A$ and $s \in S$. This map can be extended to $f_{*}: D_{B} A \rightarrow D_{B} A_{S}$.

Proposition 2.6 Consider a non-empty subset $S \subset A$. Then a B-linear $n$-bracket $\{\{-, \ldots,-\}\}$ on $A$ induces a unique B-linear $n$-bracket on $A_{S}$. If $\{\{-, \ldots,-\}\}$ is differential for $Q \in\left(D_{B} A\right)_{n}$, then the induced $B$-linear $n$-bracket is differential for $f_{*}(Q) \in\left(D_{B} A_{S}\right)_{n}$.

Proof Note that a $n$-bracket on $A_{S}$ needs to satisfy

$$
\left\{\left\{a_{1}, \ldots, a_{n-1}, s^{-1}\right\}\right\}=-s^{-1}\left\{\left\{a_{1}, \ldots, a_{n-1}, s\right\}\right\} s^{-1},
$$

for any $a_{1}, \ldots, a_{n-1} \in A_{S}$ and $s \in S$ due to the derivation property. Using the cyclic antisymmetry and the derivation property, we can then always rewrite $\left\{\left\{a_{1}, \ldots, a_{n}\right\}\right\}$ with $a_{1}, \ldots, a_{n} \in A_{S}$ in terms of sums and products in $A_{S}$ containing only the $n$-bracket evaluated on elements of $A$.

We use this result without further mention throughout the text. Next, if $e \in B$ is an idempotent, we get a canonical map $\pi^{e}: A \rightarrow e A e, a \mapsto e a e$, which extends to double derivations as $\pi_{*}^{e}: D_{A / B} \rightarrow D_{e A e / e B e}, \delta \mapsto e \delta e$. In the case where $B=B e B$, we get a non-unique decomposition $1=\sum_{i} p_{i} e q_{i}$, and it yields a trace map $\operatorname{Tr}: A \rightarrow e A e$ given by $\operatorname{Tr}(a)=\sum_{i} e q_{i} a p_{i} e$. It also gives a map $\operatorname{Tr}: D_{A / B} \rightarrow D_{e A e / e B e}$ by setting $\operatorname{Tr}(\delta)=\sum_{i} e q_{i} \delta p_{i} e$, which can be written as $\operatorname{Tr}(\delta)(e a e)=e \delta^{\prime}(a) p_{i} e \otimes e q_{i} \delta^{\prime \prime}(a) e$ for any $a \in A$. To extend this to polyvector fields, note that $\operatorname{Tr}: D_{B} A \rightarrow e D_{B} A e: Q \mapsto \sum_{i} e q_{i} Q p_{i} e$ defines a map $D_{B} A \rightarrow D_{e B e} e A e$ by Proposition 2.5 .

Proposition 2.7 Assume that $e \in B$ is an idempotent. Then a B-linear $n$-bracket $\{\{-, \ldots,-\}\}$ on $A$ induces a unique e Be-linear $n$-bracket on eAe. If $B=B e B$ and $\{\{-, \ldots,-\}\}$ is differential for $Q \in\left(D_{B} A\right)_{n}$, then the induced eBe-linear $n$-bracket is differential for $\operatorname{Tr}(Q) \in\left(D_{e B e} e A e\right)_{n}$.

Proof Fix $a_{1}, \ldots, a_{n} \in A$. Denoting $\left\{\left\{a_{1}, \ldots, a_{n}\right\}\right\}$ as $b_{1} \otimes \ldots \otimes b_{n} \in A^{\otimes n}$ (up to linear combinations), we get the unique induced $n$-bracket

$$
\left\{\left\{e a_{1} e, \ldots, e a_{n} e\right\}\right\}=(e \otimes \ldots \otimes e)\left\{\left\{a_{1}, \ldots, a_{n}\right\}\right\}(e \otimes \ldots \otimes e)=e b_{1} e \otimes \ldots \otimes e b_{n} e \in(e A e)^{\otimes n} .
$$

If the $n$-bracket is differential for $Q=\delta_{1}, \ldots, \delta_{n} \in\left(D_{B} A\right)_{n}$, we get from Eq. 2.3 and Proposition 2.1 that

$\left\{\left\{e a_{1} e, \ldots, e a_{n} e\right\}\right\}=\sum_{i=0}^{n-1}(-1)^{(n-1) i}(e \otimes \ldots \otimes e) \tau_{(1 \ldots n)}^{i}\{\{-, \ldots,-\}\}_{Q} \tau_{(1 \ldots n)}^{-i}\left(a_{1}, \ldots, a_{n}\right)(e \otimes \ldots \otimes e)$,

with $\{\{-, \ldots,-\}\}_{Q}$ given by Eq. 2.1b. Assuming that $1=\sum_{i} p_{i} e q_{i}$, we can write for $i=0$ 


$$
\begin{aligned}
& (e \otimes \ldots \otimes e)\left\{\left\{a_{1}, \ldots, a_{n}\right\} \tilde{Q}_{Q}(e \otimes \ldots \otimes e)\right. \\
= & e \delta_{n}\left(a_{n}\right)^{\prime} 1 \delta_{1}\left(a_{1}\right)^{\prime \prime} e \otimes e \delta_{1}\left(a_{1}\right)^{\prime} 1 \delta_{2}\left(a_{2}\right)^{\prime \prime} e \otimes \ldots \otimes e \delta_{n-1}\left(a_{n-1}\right)^{\prime} 1 \delta_{n}\left(a_{n}\right)^{\prime \prime} e \\
= & \sum_{i_{1}} \ldots \sum_{i_{n}} \delta_{n}\left(e a_{n} e\right)^{\prime} p_{i_{1}} e q_{i_{1}} \delta_{1}\left(e a_{1} e\right)^{\prime \prime} \otimes \ldots \otimes \delta_{n-1}\left(e a_{n-1} e\right)^{\prime} p_{i_{n}} e q_{i_{n}} \delta_{n}\left(e a_{n} e\right)^{\prime \prime} \\
= & \sum_{i_{1}} \ldots \sum_{i_{n}}\left\{\left\{e a_{1} e, \ldots, e a_{n} e\right\}\right\}_{e q_{i_{1}} \delta_{1} p_{i_{2}} e q_{i_{2}} \delta_{2} p_{i_{3}} e \ldots e q_{i_{n}} \delta_{n} p_{i_{1}} e} \\
= & \sum_{i_{1}}\left\{\left\{e a_{1} e, \ldots, e a_{n} e\right\} \tilde{\}}_{e q_{i_{1}} \delta_{1} \delta_{2} \ldots \delta_{n} p_{i_{1}} e}=\left\{\left\{e a_{1} e, \ldots, e a_{n} e\right\} \tilde{\}}_{\operatorname{Tr}\left(\delta_{1} \delta_{2} \ldots \delta_{n}\right)} .\right.\right.
\end{aligned}
$$

The argument is similar for $i=1, \ldots, n-1$ so that

$$
\left\{\left\{e a_{1} e, \ldots, e a_{n} e\right\}\right\}=\sum_{i=0}^{n-1}(-1)^{(n-1) i} \tau_{(1 \ldots n)}^{i}\left\{\{-, \ldots,-\} \tilde{\}}_{\operatorname{Tr}\left(\delta_{1} \delta_{2} \ldots \delta_{n}\right)} \tau_{(1 \ldots n)}^{-i}\left(e a_{1} e, \ldots, e a_{n} e\right),\right.
$$

which is differential for $\operatorname{Tr}\left(\delta_{1} \delta_{2} \ldots \delta_{n}\right)$ by definition.

Next, consider algebras $A$ and $A^{\prime}$ respectively over $B$ and $B^{\prime}$. We get that $A \oplus A^{\prime}$ is a $\left(B \oplus B^{\prime}\right.$ )-algebra, and we can identify $D_{A \oplus A^{\prime} / B \oplus B^{\prime}}$ with $D_{A / B} \oplus D_{A^{\prime} / B^{\prime}}$. This extends to the identification of $D_{B \oplus B^{\prime}} A \oplus A^{\prime}$ and $D_{B} A \oplus D_{B^{\prime}} A^{\prime}$.

Proposition 2.8 Assume that $\{\{-, \ldots,-\}\}$ is a B-linear $n$-bracket on $A$, and $\{\{-, \ldots,-\}\}^{\prime}$ is a $B^{\prime}$-linear $n$-bracket on $A^{\prime}$. Then there exists a unique $\left(B \oplus B^{\prime}\right)$-linear $n$-bracket $\{\{-, \ldots,-\}\}^{\oplus}$ on $A \oplus A^{\prime}$ extending the $n$-brackets $\{\{-, \ldots,-\}\}$ and $\{\{-, \ldots,-\}\}^{\prime}$, while it is such that $\left\{\left\{c_{1}, \ldots, c_{n}\right\}\right\}^{\oplus}=0$ whenever there exists $i \neq j$ with $c_{i}=(a, 0), c_{j}=(0, b)$. Furthermore, if the $n$-brackets on $A$ and $A^{\prime}$ are differential for $Q \in\left(D_{B} A\right)_{n}$ and $Q^{\prime} \in\left(D_{B^{\prime}} A^{\prime}\right)_{n}$, then $\{\{-, \ldots,-\}\}^{\oplus}$ is differential for $\left(Q, Q^{\prime}\right) \in\left(D_{B \oplus B^{\prime}} A \oplus A^{\prime}\right)_{n}$.

Proof It follows directly by linearity since

$$
\begin{aligned}
\left\{\left\{\left(a_{1}, b_{1}\right), \ldots,\left(a_{n}, b_{n}\right)\right\}\right\}^{\oplus} & =\left\{\left\{\left(a_{1}, 0\right), \ldots,\left(a_{n}, 0\right)\right\}\right\}^{\oplus}+\left\{\left\{\left(0, b_{1}\right), \ldots,\left(0, b_{n}\right)\right\}\right\}^{\oplus} \\
& =\left(\left\{\left\{a_{1}, \ldots, a_{n}\right\}\right\}, 0\right)+\left(0,\left\{\left\{b_{1}, \ldots, b_{n}\right\}\right\}^{\prime}\right),
\end{aligned}
$$

for any $a_{1}, \ldots, a_{n} \in A, b_{1}, \ldots, b_{n} \in A^{\prime}$.

Given algebras $A, A^{\prime}$ over $B$ with algebra monomorphisms $j: B \rightarrow A$ and $j^{\prime}: B \rightarrow A^{\prime}$, recall that the free algebra $A *_{B} A^{\prime}$ is given by $T_{\mathbb{k}}\left(A \oplus A^{\prime}\right) / J$, where $J$ is the two-sided ideal generated by the relations $a_{1} \otimes a_{2}=a_{1} a_{2}, a_{1}^{\prime} \otimes a_{2}^{\prime}=a_{1}^{\prime} a_{2}^{\prime}, j(b)=j^{\prime}(b)$ for all $a_{1}, a_{2} \in A$, $a_{1}^{\prime}, a_{2}^{\prime} \in A^{\prime}$ and $b \in B$. Set $\bar{A}=A *_{B} A^{\prime}$. The canonical maps $i: A \rightarrow \bar{A}, i^{\prime}: A^{\prime} \rightarrow \bar{A}$ yield maps of double derivations $i_{*}: D_{A / B} \rightarrow D_{\bar{A} / A^{\prime}}$ and $i_{*}^{\prime}: D_{A^{\prime} / B} \rightarrow D_{\bar{A} / A}$, which can both be seen to take value in $D_{\bar{A} / B}$. In particular, they extend to polyvector fields.

Proposition 2.9 Assume that $\{\{-, \ldots,-\}\}$ and $\{\{-, \ldots,-\}\}^{\prime}$ are B-linear $n$-brackets on $A$ and $A^{\prime}$ respectively. Then there exists a unique $n$-bracket $\{\{-, \ldots,-\}\}^{*}$ on $\bar{A}=A *_{B} A^{\prime}$ extending the $n$ brackets $\{\{-, \ldots,-\}\}$ and $\{\{-, \ldots,-\}\}^{\prime}$, while it is such that $\left\{\left\{a_{1}, \ldots, a_{n}\right\}\right\}^{*}=0$ whenever there exists $i \neq j$ with $a_{i} \in A, a_{j} \in A^{\prime}$. Furthermore, if the $n$-brackets on $A$ and $A^{\prime}$ are differential for $Q \in\left(D_{B} A\right)_{n}$ and $Q^{\prime} \in\left(D_{B} A^{\prime}\right)_{n}$, then $\{\{-, \ldots,-\}\}^{*}$ is differential for $i_{*}(Q)+i_{*}^{\prime}\left(Q^{\prime}\right) \in\left(D_{B} \bar{A}\right)_{n}$.

Endowing $A^{\prime}$ with the zero $n$-bracket, we get the next result.

Corollary 2.10 Assume that $\{\{-, \ldots,-\}\}$ is a B-linear $n$-bracket on A. Then there is a unique $A^{\prime}$-linear $n$-bracket on $\bar{A}=A *_{B} A^{\prime}$ extending it. If $\{\{-, \ldots,-\}\}$ is differential for $Q \in\left(D_{B} A\right)_{n}$, then the induced $A^{\prime}$-linear $n$-bracket is differential for $i_{*}(Q) \in\left(D_{A^{\prime}} \bar{A}\right)_{n}$. 
In particular, $n$-brackets are compatible with base changes.

We now use these results, and assume that there exist orthogonal idempotents $e_{1}, e_{2} \in B$. The extension algebra $\bar{A}$ of $A$ along the pair $\left(e_{1}, e_{2}\right)$ is given by

$$
\bar{A}=A *_{\mathbb{k} e_{1} \oplus \mathbb{k} e_{2} \oplus \mathbb{k} \mu}\left(\mathrm{Mat}_{2}(\mathbb{k}) \oplus \mathbb{k} \mu\right)=A *_{B} \bar{B},
$$

where $\mu=1-e_{1}-e_{2}$, and $\operatorname{Mat}_{2}(\mathbb{k})$ is seen as the $\mathbb{k}$-algebra generated by $e_{1}=$ $e_{11}, e_{12}, e_{21}, e_{2}=e_{22}$ with $e_{s t} e_{u v}=\delta_{t u} e_{s v}$. The fusion algebra $A^{f}$ of $A$ along $\left(e_{1}, e_{2}\right)$ is the algebra obtained from $\bar{A}$ by discarding elements of $e_{2} \bar{A}+\bar{A} e_{2}$, i.e.

$$
A^{f}=\epsilon \bar{A} \epsilon, \quad \text { for } \epsilon=1-e_{2} .
$$

We also say that $A^{f}$ is the fusion algebra obtained by fusing $e_{2}$ onto $e_{1}$. Note that $A^{f}$ is a $B^{f}$-algebra for $B^{f}=\epsilon \bar{B} \epsilon$. The elements of $A^{f}$ can be characterised in terms of generators as follows. (This choice of generators was considered by Van den Bergh [20, Proof of Lemma 5.3.3].)

Lemma 2.11 Elements of $A^{f}$ can be written in terms of generators of the following forms

$\begin{array}{rcc}\text { (first type) } & a=t, & t \in \epsilon A \epsilon, \\ \text { (second type) } & a=e_{12} u, & u \in e_{2} A \epsilon, \\ \text { (third type) } & a=v e_{21}, & v \in \epsilon A e_{2}, \\ \text { (fourth type) } & a=e_{12} w e_{21}, & w \in e_{2} A e_{2} .\end{array}$

Remark that $\bar{B}$ satisfies $\bar{B}=\bar{B} \epsilon \bar{B}$ since $1=1 \epsilon 1+e_{21} \epsilon e_{12}$. Using the map $\operatorname{Tr}: D_{\bar{B}} \bar{A} \rightarrow$ $D_{B^{f}} A^{f}$ given by $\operatorname{Tr}(\bar{Q})=\epsilon \bar{Q} \epsilon+\epsilon e_{12} \bar{Q} e_{21} \epsilon$ together with $i_{*}: D_{B} A \rightarrow D_{\bar{B}} \bar{A}$, we get a map Tro $i_{*}: D_{B} A \rightarrow D_{B} A^{f}$. We combine Corollary 2.10 and Proposition 2.7 to get the following generalisation of [20, Corollary 2.5.6].

Proposition 2.12 If $A$ is a $B$-algebra with $n$-bracket $\{\{-, \ldots,-\}\}$, it induces $n$-brackets on $\bar{A}$ over $\bar{B}$ and $A^{f}$ over $B^{f}$. If the $n$-bracket on $A$ is differential for $Q \in\left(D_{B} A\right)_{n}$, then the induced $n$-brackets are differential for $i_{*}(Q) \in\left(D_{\bar{B}} \bar{A}\right)_{n}$ and $\operatorname{Tr} \circ i_{*}(Q) \in\left(D_{B^{f}} A^{f}\right)_{n}$ respectively.

From now on, we denote the compositions $\operatorname{Tr} \circ i$ and $\operatorname{Tr} \circ i_{*}$ simply as $\operatorname{Tr}$.

\subsubsection{Double quasi-Poisson brackets from the gauge elements}

Assume that $B=\mathbb{k} e_{1} \oplus \ldots \oplus \mathbb{k} e_{N}$, where the $\left(e_{s}\right)$ form a complete set of orthogonal idempotents. We define for all $s=1, \ldots, N$ a double derivation $E_{s} \in D_{A / B}$ such that for any $a \in A$, $E_{s}(a)=a e_{s} \otimes e_{s}-e_{s} \otimes e_{s} a$. These are called the gauge elements. Following [20, §5.1], we say that a double bracket $\{\{-,-\}\}$ on $A$ over $B$ is quasi-Poisson if it satisfies

$$
\{\{-,-,-\}\}=\frac{1}{12} \sum_{s=1}^{N}\{\{-,-,-\}\}_{E_{s}^{3}},
$$

where on the left-hand side we have the associated triple bracket given by Eq. 1.4, while the triple brackets in the right-hand side are defined from Proposition 2.1 with $E_{s}^{3} \in\left(D_{B} A\right)_{3}$. It is then an easy exercise to check that Eq. 2.7 evaluated on $a, b, c \in A$ gives (1.6), so that this definition coincides with the one given in the introduction. Note that under the assumption of Proposition 2.2 the double quasi-Poisson bracket $\{\{-,-\}\}$ is differential for some $Q \in\left(D_{B} A\right)_{2}$, and we get the equivalent condition that $\{Q, Q\}_{\mathrm{SN}}=\frac{1}{6} \sum_{s=1}^{N} E_{s}^{3}$ modulo $\left[D_{B} A, D_{B} A\right]$ by Propositions 2.1 and 2.4. 
In a double quasi-Poisson algebra $(A,\{\{-,-\}\})$, we say that an element $\Phi \in A^{\times}$is a moment map if $\Phi_{s}=e_{s} \Phi e_{s}$ satisfies $\left\{\left\{\Phi_{s},-\right\}\right\}=\frac{1}{2}\left(\Phi_{s} E_{s}+E_{s} \Phi_{s}\right)$ for all $s=1, \ldots, N$. It is an easy exercise to check that the $s$-th condition is equivalent to Eq. 1.7, hence this definition of moment map is equivalent to the one given in the introduction.

Remark 2.13 Assume that $B=\mathbb{k} e_{1} \oplus \ldots \oplus \mathbb{k} e_{N}, B^{\prime}=\mathbb{k} e_{1}^{\prime} \oplus \ldots \oplus \mathbb{k} e_{M}^{\prime}$, and we have double quasi-Poisson brackets $\{\{-,-\}\}$ and $\{\{-,-\}\}^{\prime}$ over $A$ and $A^{\prime}$ respectively. Then $\{\{-,-\}\}^{\oplus}$ is a $\left(B \oplus B^{\prime}\right)$-linear double quasi-Poisson bracket over $A \oplus A^{\prime}$. This can be obtained by combining Proposition 2.8 and the definition of double quasi-Poisson bracket using the gauge elements given above. Moreover, if $\Phi$ and $\Phi^{\prime}$ are the corresponding moment maps, then $\left(\Phi, \Phi^{\prime}\right)$ turns $A \oplus A^{\prime}$ into a quasi-Hamiltonian algebra.

\subsection{Main theorems}

Hereafter, we assume that $A$ is a $B$-algebra for $B=\mathbb{k} e_{1} \oplus \ldots \oplus \mathbb{k} e_{N}$ a semisimple $\mathbb{k}$-algebra. Our aim is to prove the following results.

Theorem 2.14 Assume that $(A,\{\{-,-\}\})$ is a double quasi-Poisson algebra over $B$. Consider the fusion algebra $A^{f}$ obtained by fusing $e_{2}$ onto $e_{1}$. Then, $A^{f}$ has a $B^{f}$-linear double quasi-Poisson bracket given by

$$
\{\{-,-\}\}^{f}:=\{\{-,-\}\}+\{\{-,-\}\}_{\text {fus }},
$$

where the first double bracket on the right-hand side is induced in $A^{f}$ by the one of $A$ using Proposition 2.12, and the second double bracket $\{\{-,-\}\}_{\text {fus }}$ is defined by $-\frac{1}{2} \operatorname{Tr}\left(E_{1}\right) \operatorname{Tr}\left(E_{2}\right) \in$ $\left(D_{B^{f}} A^{f}\right)_{2}$ using Proposition 2.1.

Theorem 2.15 Assume that $(A,\{\{-,-\}\}, \Phi)$ is a quasi-Hamiltonian algebra over $B$, where $\Phi=$ $\sum_{s} \Phi_{s} \in \oplus_{s} e_{s} A e_{s}$. Consider the fusion algebra $A^{f}$ obtained by fusing $e_{2}$ onto $e_{1}$. Then $A^{f}$ is a quasi-Hamiltonian algebra for the double quasi-Poisson bracket $\{\{-,-\}\}^{f}$ given in Theorem 2.14 and for the multiplicative moment map

$$
\Phi^{f}=e_{1} \operatorname{Tr}\left(\Phi_{1}\right) \operatorname{Tr}\left(\Phi_{2}\right) e_{1}+\sum_{s \neq 1,2} e_{s} \operatorname{Tr}\left(\Phi_{s}\right) e_{s} .
$$

Remark 2.16 In the case where the double quasi-Poisson bracket $\{\{-,-\}\}$ is differential for some $Q \in\left(D_{B} A\right)_{2}$, we have that the double quasi-Poisson bracket (2.8) is differential for $Q^{f}:=\operatorname{Tr}(Q)-\frac{1}{2} \operatorname{Tr}\left(E_{1}\right) \operatorname{Tr}\left(E_{2}\right)$ by Proposition 2.12 and linearity of the map $\mu$ in Proposition 2.1. Therefore, Theorems 2.14 and 2.15 are nothing else than [20, Theorems 5.3.1,5.3.2] in such a case. However, if the double quasi-Poisson bracket is not differential (which can only happen if $A$ does not satisfy the assumptions from Proposition 2.2), these results extend their analogues proved in the differential case, as expected by Van den Bergh [20, §5.3].

\subsection{Preparation for the proofs}

\subsubsection{Image of the gauge elements}

We have well-defined double derivations $E_{s} \in D_{A / B}, 1 \leq s \leq N$, and we want to know what are their images in the fusion algebra $A^{f}$, obtained by fusing the idempotent $e_{2}$ onto $e_{1}$ as in $\$ 2.1 .2$. To avoid any conflicting notations, write $E_{1}, E_{2}, \ldots, E_{N}$ for the gauge elements over $A$ and their image under $D_{A / B} \rightarrow D_{\bar{A} / \bar{B}}$, and let $F_{1}, F_{3}, \ldots, F_{N}$ be the gauge elements in $D_{A^{f} / B^{f}}$, with $B^{f}=\mathbb{k} e_{1} \oplus \mathbb{k} e_{3} \oplus \ldots \oplus \mathbb{k} e_{N}$. We now relate the double derivations $\operatorname{Tr} E_{s}$ and $F_{s}$. (These results first appeared in $[20, \S 5.3]$, but we give a proof for the sake of clarity.) 
Lemma 2.17 For any $s \neq 1,2, \operatorname{Tr}\left(E_{s}\right)=F_{s}$.

Proof We only need to prove the equality on generators of $A^{f}$. By Lemma 2.11, we can write any $a \in A^{f}$ as $a=e_{+} \alpha e_{-}$, for $a \in A$ and some $e_{+} \in\left\{e_{12}, \epsilon\right\}, e_{-} \in\left\{e_{21}, \epsilon\right\}$. Hence, by definition of gauge element and the trace map

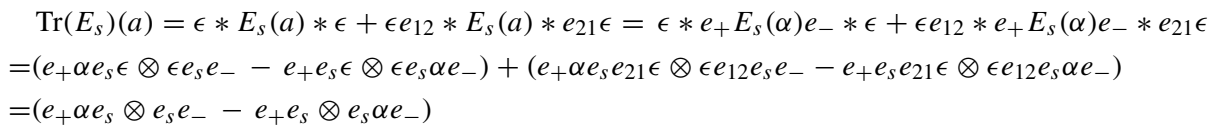

since $e_{s} \epsilon=e_{s}=\epsilon e_{s}$ and $e_{s} e_{21}=0=e_{12} e_{s}$ as $s \neq 2$. Now, remark that we can write this as

$$
\operatorname{Tr}\left(E_{S}\right)(a)=\left(e_{+} \alpha e_{-} e_{s}\right) \otimes e_{s}-e_{s} \otimes e_{s}\left(e_{+} \alpha e_{-}\right) .
$$

Indeed, for the first term, either $e_{-}=\epsilon$ and $e_{s} e_{-}=e_{s}=e_{-} e_{s}$, or $e_{-} \neq \epsilon$ and $e_{s} e_{-}=0=e_{-} e_{s}$. The same applies to the second term.

Lemma 2.18 The double derivations $\operatorname{Tr}\left(E_{1}\right), \operatorname{Tr}\left(E_{2}\right)$ take the following forms on generators : if $a=t$ for $t \in \epsilon A \epsilon$,

$$
\operatorname{Tr}\left(E_{1}\right)(t)=t e_{1} \otimes e_{1}-e_{1} \otimes e_{1} t, \quad \operatorname{Tr}\left(E_{2}\right)(t)=0,
$$

if $a=e_{12} u$ for $u \in e_{2} A \epsilon$,

$$
\operatorname{Tr}\left(E_{1}\right)\left(e_{12} u\right)=\left(e_{12} u\right) e_{1} \otimes e_{1}, \quad \operatorname{Tr}\left(E_{2}\right)\left(e_{12} u\right)=-e_{1} \otimes\left(e_{12} u\right),
$$

if $a=v e_{21}$ for $v \in \epsilon A e_{2}$,

$$
\operatorname{Tr}\left(E_{1}\right)\left(v e_{21}\right)=-e_{1} \otimes e_{1}\left(v e_{21}\right), \quad \operatorname{Tr}\left(E_{2}\right)\left(v e_{21}\right)=\left(v e_{21}\right) \otimes e_{1},
$$

if $a=e_{12} w e_{21}$ for $w \in e_{2} A e_{2}$,

$$
\operatorname{Tr}\left(E_{1}\right)\left(e_{12} w e_{21}\right)=0, \quad \operatorname{Tr}\left(E_{2}\right)\left(e_{12} w e_{21}\right)=\left(e_{12} w e_{21}\right) e_{1} \otimes e_{1}-e_{1} \otimes e_{1}\left(e_{12} w e_{21}\right) .
$$

In particular, $\operatorname{Tr}\left(E_{1}\right)+\operatorname{Tr}\left(E_{2}\right)=F_{1}$.

Proof First, remark that $\operatorname{Tr}\left(E_{1}\right)=\epsilon E_{1} \epsilon$ and $\operatorname{Tr}\left(E_{2}\right)=\epsilon e_{12} E_{2} e_{21} \epsilon$, by expansion as in Lemma 2.17 or using that in $D_{B} A$ we have $E_{s} \in e_{s} D_{B} A e_{s}$. Therefore, writing a generator $a \in A^{f}$ as $a=e_{+} \alpha e_{-}$as in Lemma 2.17 ,

$$
\begin{aligned}
& \operatorname{Tr}\left(E_{1}\right)(a)=e_{+} \alpha e_{1} \otimes e_{1} e_{-}-e_{+} e_{1} \otimes e_{1} \alpha e_{-}, \\
& \operatorname{Tr}\left(E_{2}\right)(a)=e_{+} \alpha e_{21} \otimes e_{12} e_{-}-e_{+} e_{21} \otimes e_{12} \alpha e_{-},
\end{aligned}
$$

using the relations between idempotents. In the first case (2.6a), $\alpha=t, e_{+}=e_{-}=\epsilon$ so that the identities are clear. In the second case (2.6b) with $\alpha=u, e_{+}=e_{12}$ and $e_{-}=\epsilon$ so that

$$
\operatorname{Tr}\left(E_{1}\right)(a)=e_{12} u e_{1} \otimes e_{1}-e_{12} e_{1} \otimes e_{1} u, \quad \operatorname{Tr}\left(E_{2}\right)(a)=e_{12} u e_{21} \otimes e_{1}-e_{1} \otimes e_{12} u,
$$

and we get our claim by remarking that $e_{12} e_{1}=0$ and $u e_{21}=u \epsilon e_{21}=0$. In the third case (2.6c) we take $\alpha=v, e_{+}=\epsilon$ and $e_{-}=e_{21}$, which yields

$$
\operatorname{Tr}\left(E_{1}\right)(a)=v e_{1} \otimes e_{1} e_{21}-e_{1} \otimes e_{1} v e_{21}, \quad \operatorname{Tr}\left(E_{2}\right)(a)=v e_{21} \otimes e_{1}-\epsilon e_{21} \otimes e_{12} v e_{21} .
$$

Hence, it suffices to remark that $e_{1} e_{21}=0$ and $e_{12} v=e_{12} \epsilon v=0$. Finally for Eq. 2.6d, we take $\alpha=w$ and $e_{+}=e_{12}, e_{-}=e_{21}$ to get

$\operatorname{Tr}\left(E_{1}\right)(a)=e_{12} w e_{1} \otimes e_{1} e_{21}-e_{12} e_{1} \otimes e_{1} w e_{21}, \quad \operatorname{Tr}\left(E_{2}\right)(a)=e_{12} w e_{21} \otimes e_{12} e_{21}-\epsilon e_{12} e_{21} \otimes e_{12} w e_{21}$, so that our claim follows since $e_{1} e_{21}=0=e_{12} e_{1}$. 


\subsubsection{Properties of the double bracket $\{\{-,-\}\}_{\text {fus }}$}

Recall that the double bracket $\{\{-,-\}\}_{f u s}$ is defined by $-\frac{1}{2} \operatorname{Tr}\left(E_{1}\right) \operatorname{Tr}\left(E_{2}\right) \in\left(D_{B^{f}} A^{f}\right)_{2}$ using Proposition 2.1.

Lemma 2.19 On generators of $A^{f}$, the double bracket $\{\{-,-\}\}_{f u s}$ can be written as

$$
\begin{aligned}
& \{\{\epsilon t \epsilon, \epsilon \tilde{t} \epsilon\}\}_{f u s}=0, \\
& \left\{\left\{\epsilon t \epsilon, e_{12} u \epsilon\right\}\right\}_{f u s}=\frac{1}{2}\left(e_{1} \otimes t e_{12} u-e_{1} t \otimes e_{12} u\right), \\
& \left\{\left\{\epsilon t \epsilon, \epsilon v e_{21}\right\}\right\}_{f u s}=\frac{1}{2}\left(v e_{21} t \otimes e_{1}-v e_{21} \otimes t e_{1}\right), \\
& \left\{\left\{\epsilon t \epsilon, e_{12} w e_{21}\right\}\right\}_{f u s}=\frac{1}{2}\left(e_{12} w e_{21} t \otimes e_{1}+e_{1} \otimes t e_{12} w e_{21}-e_{12} w e_{21} \otimes t e_{1}-e_{1} t \otimes e_{12} w e_{21}\right),
\end{aligned}
$$

when the first component $\epsilon t \epsilon$ is a generator of the first type (2.6a),

$$
\begin{aligned}
& \left\{\left\{e_{12} u \epsilon, \epsilon t \epsilon\right\}\right\}_{f u s}=\frac{1}{2}\left(e_{12} u \otimes e_{1} t-t e_{12} u \otimes e_{1}\right), \\
& \left\{\left\{e_{12} u \epsilon, e_{12} \tilde{u} \epsilon\right\}\right\}_{f u s}=\frac{1}{2}\left(e_{1} \otimes e_{12} u e_{12} \tilde{u}-e_{12} \tilde{u} e_{12} u \otimes e_{1}\right), \\
& \left\{\left\{e_{12} u \epsilon, \epsilon v e_{21}\right\}\right\}_{f u s}=\frac{1}{2}\left(e_{12} u \otimes e_{1} v e_{21}-v e_{21} \otimes e_{12} u e_{1}\right), \\
& \left\{\left\{e_{12} u \epsilon, e_{12} w e_{21}\right\}\right\}_{f u s}=\frac{1}{2}\left(e_{1} \otimes e_{12} u e_{12} w e_{21}-e_{12} w e_{21} \otimes e_{12} u e_{1}\right),
\end{aligned}
$$

when the first component $e_{12} u \epsilon$ is a generator of the second type (2.6b),

$$
\begin{aligned}
& \left\{\left\{\epsilon v e_{21}, \epsilon t \epsilon\right\}\right\}_{f u s}=\frac{1}{2}\left(t e_{1} \otimes v e_{21}-e_{1} \otimes v e_{21} t\right), \\
& \left\{\left\{\epsilon v e_{21}, e_{12} u \epsilon\right\}\right\}_{f u s}=\frac{1}{2}\left(e_{12} u e_{1} \otimes v e_{21}-e_{1} v e_{21} \otimes e_{12} u\right), \\
& \left\{\left\{\epsilon v e_{21}, \epsilon \tilde{v} e_{21}\right\}\right\}_{f u s}=\frac{1}{2}\left(\tilde{v} e_{21} v e_{21} \otimes e_{1}-e_{1} \otimes v e_{21} \tilde{v} e_{21}\right), \\
& \left\{\left\{\epsilon v e_{21}, e_{12} w e_{21}\right\}\right\}_{f u s}=\frac{1}{2}\left(e_{12} w e_{21} v e_{21} \otimes e_{1}-e_{1} v e_{21} \otimes e_{12} w e_{21}\right),
\end{aligned}
$$

when the first component $\epsilon$ ve 21 is a generator of the third type (2.6c),

$$
\begin{aligned}
& \left\{\left\{e_{12} w e_{21}, \epsilon t \epsilon\right\}\right\}_{f u s}=\frac{1}{2}\left(t e_{1} \otimes e_{12} w e_{21}+e_{12} w e_{21} \otimes e_{1} t-t e_{12} w e_{21} \otimes e_{1}-e_{1} \otimes e_{12} w e_{21} t\right), \\
& \left\{\left\{e_{12} w e_{21}, e_{12} u \epsilon\right\}\right\}_{f u s}=\frac{1}{2}\left(e_{12} u e_{1} \otimes e_{12} w e_{21}-e_{12} u e_{12} w e_{21} \otimes e_{1}\right) \\
& \left\{\left\{e_{12} w e_{21}, \epsilon v e_{21}\right\}\right\}_{f u s}=\frac{1}{2}\left(e_{12} w e_{21} \otimes e_{1} v e_{21}-e_{1} \otimes e_{12} w e_{21} v e_{21}\right) \\
& \left\{\left\{e_{12} w e_{21}, e_{12} \tilde{w} e_{21}\right\}\right\}_{f u s}=0
\end{aligned}
$$

when the first component $e_{12} w e_{21}$ is a generator of the fourth type (2.6d).

Proof Remark that from the definition of the double bracket $\{\{-,-\}\}_{f u s}$ together with Eq. $2.1 \mathrm{~b}$ we can write

$$
\begin{aligned}
\{\{a, b\}\}_{f u s}= & -\frac{1}{2} \operatorname{Tr}\left(E_{2}\right)(b)^{\prime} \operatorname{Tr}\left(E_{1}\right)(a)^{\prime \prime} \otimes \operatorname{Tr}\left(E_{1}\right)(a)^{\prime} \operatorname{Tr}\left(E_{2}\right)(b)^{\prime \prime} \\
& +\frac{1}{2} \operatorname{Tr}\left(E_{1}\right)(b)^{\prime} \operatorname{Tr}\left(E_{2}\right)(a)^{\prime \prime} \otimes \operatorname{Tr}\left(E_{2}\right)(a)^{\prime} \operatorname{Tr}\left(E_{1}\right)(b)^{\prime \prime} .
\end{aligned}
$$


It remains to use Eqs. 2.10-2.13 to get the required identities. For example, to get Eq. 2.14b we find from Eqs. 2.10 and 2.11

$$
\begin{aligned}
\left\{\left\{\epsilon t \epsilon, e_{12} u \epsilon\right\}\right\}_{f u s} & =-\frac{1}{2} \operatorname{Tr}\left(E_{2}\right)\left(e_{12} u\right)^{\prime} \operatorname{Tr}\left(E_{1}\right)(t)^{\prime \prime} \otimes \operatorname{Tr}\left(E_{1}\right)(t)^{\prime} \operatorname{Tr}\left(E_{2}\right)\left(e_{12} u\right)^{\prime \prime} \\
& =-\frac{1}{2}\left(-e_{1} e_{1} \otimes t e_{1} e_{12} u+e_{1} e_{1} t \otimes e_{1} e_{12} u\right)=\frac{1}{2} e_{1} \otimes t e_{12} u-\frac{1}{2} e_{1} t \otimes e_{12} u .
\end{aligned}
$$

The exact same method works in each case. Note that only ten cases need to be computed as other double brackets can be obtained by cyclic antisymmetry : $\{\{b, a\}\}_{f u s}=-\{\{a, b\}\}_{f u s}^{\prime \prime} \otimes$ $\{\{a, b\}\}_{f u s}^{\prime}$.

These explicit forms of the double bracket $\{\{-,-\}\}_{f u s}$ are central in the proof of the next result, which we postpone to Appendix A.

Lemma 2.20 Assume that $\{\{-,-\}\}$ is an arbitrary B-linear double bracket on A. Consider the induced $B^{f}$-linear double bracket $\{\{-,-\}\}$ on $A^{f}$, and define the double bracket $\{\{-,-\}\}_{\text {fus }}$ as in Theorem 2.14. Furthermore, set $\{\{-,-\}\}^{f}:=\{\{-,-\}\}+\{\{-,-\}\}_{\text {fus }}$. Then the $B^{f}$-linear map $\kappa:\left(A^{f}\right)^{\times 3} \rightarrow\left(A^{f}\right)^{\otimes 3}$ defined by

$$
\kappa(-,-,-)=\{\{-,-,-\}\}^{f}-\{\{-,-,-\}\}-\{\{-,-,-\}\}_{\text {fus }},
$$

vanishes. (Here, the induced triple brackets on the right-hand side are given by Eq. 1.4 using $\{\{-,-\}\}^{f},\{\{-,-\}\}$ and $\{\{-,-\}\}_{\text {fus }}$ respectively.)

\subsection{Fusion for the double quasi-Poisson bracket}

We prove Theorem 2.14. To do so, we need to show that $\{\{-,-,-\}\}^{f}=$ $\frac{1}{12} \sum_{s \neq 2}\{\{-,-,-\}\}_{F_{i}^{3}}$, where $\{\{-,-,-\}\}^{f}$ is the triple bracket associated to the double bracket defined by Eq. 2.8. By Lemma 2.20, we simply have that

$$
\{\{-,-,-\}\}^{f}=\{\{-,-,-\}\}+\{\{-,-,-\}\}_{\text {fus }} .
$$

By assumption, $\{\{-,-\}\}$ is quasi-Poisson in $A$, hence $\{\{-,-,-\}\}$ coincides with the differential double bracket defined by $\frac{1}{12} \sum_{s} E_{s}^{3} \in\left(D_{B} A\right)_{3}$, see $\$ 2.1 .3$. We get from Proposition 2.12 that we can write $\{\{-,-,-\}\}=\frac{1}{12} \sum_{s}\{\{-,-,-\}\}_{\operatorname{Tr}\left(E_{s}^{3}\right)}$ in $A^{f}$.

We rewrite each $\operatorname{Tr}\left(E_{s}^{3}\right)$ in terms of the gauge elements $F_{s}, s \neq 2$, of $A^{f}$. Since $E_{s}=$ $e_{s} E_{s} e_{s}$

$$
\operatorname{Tr}\left(E_{s}^{3}\right)=\epsilon E_{s}^{3} \epsilon=\left(\epsilon E_{s} \epsilon\right)^{3}=F_{s}^{3},
$$

for any $s \neq 1,2$ by Lemma 2.17. Similarly, since $e_{2}=e_{21} \epsilon e_{12}$,

$$
\operatorname{Tr}\left(E_{1}^{3}\right)+\operatorname{Tr}\left(E_{2}^{3}\right)=\epsilon E_{1}^{3} \epsilon+\epsilon e_{12} E_{2}^{3} e_{21} \epsilon=\left(\epsilon E_{1} \epsilon\right)^{3}+\left(\epsilon e_{12} E_{2} e_{21} \epsilon\right)^{3} .
$$

Modulo graded commutators, we can write

$$
\operatorname{Tr}\left(E_{1}^{3}\right)+\operatorname{Tr}\left(E_{2}^{3}\right)=\left[\operatorname{Tr}\left(E_{1}\right)+\operatorname{Tr}\left(E_{2}\right)\right]^{3}-3 \operatorname{Tr}\left(E_{1}\right) \operatorname{Tr}\left(E_{2}\right)^{2}-3 \operatorname{Tr}\left(E_{1}\right)^{2} \operatorname{Tr}\left(E_{2}\right),
$$

which is $F_{1}^{3}-3 \operatorname{Tr}\left(E_{1}\right) \operatorname{Tr}\left(E_{2}\right)^{2}-3 \operatorname{Tr}\left(E_{1}\right)^{2} \operatorname{Tr}\left(E_{2}\right)$ using Lemma 2.18. By Proposition 2.1, the map $\mu$ defines $n$-brackets modulo graded commutators in $D_{B^{f}} A^{f}$ so that

$\{\{-,-,-\}\}^{f}=\frac{1}{12} \sum_{s \neq 2}\{\{-,-,-\}\}_{F_{i}^{3}}-\frac{1}{4}\{\{-,-,-\}\}_{\operatorname{Tr}\left(E_{1}\right) \operatorname{Tr}\left(E_{2}\right)^{2}+\operatorname{Tr}\left(E_{1}\right)^{2} \operatorname{Tr}\left(E_{2}\right)}+\{\{-,-,-\}\}_{f u s}$.

Now, by Proposition 2.4, the bracket $\{\{-,-,-\}\}_{f u s}$ is defined by $\frac{1}{8}\left\{\operatorname{Tr}\left(E_{1}\right) \operatorname{Tr}\left(E_{2}\right), \operatorname{Tr}\left(E_{1}\right) \operatorname{Tr}\left(E_{2}\right)\right\}_{\mathrm{SN}}$. After a short computation (given e.g. in [20, §5.3]), we find that 


$$
\left\{\operatorname{Tr}\left(E_{1}\right) \operatorname{Tr}\left(E_{2}\right), \operatorname{Tr}\left(E_{1}\right) \operatorname{Tr}\left(E_{2}\right)\right\}_{\mathrm{SN}}=2 \operatorname{Tr}\left(E_{1}\right)^{2} \operatorname{Tr}\left(E_{2}\right)+2 \operatorname{Tr}\left(E_{1}\right) \operatorname{Tr}\left(E_{2}\right)^{2},
$$

modulo graded commutators, which finishes the proof.

\subsection{Fusion for the moment map}

Note that $\Phi^{f}$ has an inverse

$$
\left(\Phi^{f}\right)^{-1}=e_{1} \operatorname{Tr}\left(\Phi_{2}^{-1}\right) \operatorname{Tr}\left(\Phi_{1}^{-1}\right) e_{1}+\sum_{s \neq 1,2} e_{s} \operatorname{Tr}\left(\Phi_{s}^{-1}\right) e_{s}
$$

so that Theorem 2.15 directly follows from the following lemma.

Lemma 2.21 Assume that $s \neq 1,2$. Then for any $a \in A^{f}$

$$
\left\{\left\{\operatorname{Tr}\left(\Phi_{s}\right), a\right\}\right\}^{f}=\frac{1}{2}\left(a e_{s} \otimes \operatorname{Tr}\left(\Phi_{s}\right)+a \operatorname{Tr}\left(\Phi_{s}\right) \otimes e_{s}-e_{s} \otimes \operatorname{Tr}\left(\Phi_{s}\right) a-\operatorname{Tr}\left(\Phi_{s}\right) \otimes e_{s} a\right) .
$$

If we set $\Phi_{1}^{f}=\operatorname{Tr}\left(\Phi_{1}\right) \operatorname{Tr}\left(\Phi_{2}\right)$, we have for any $a \in A^{f}$

$$
\left\{\left\{\Phi_{1}^{f}, a\right\}\right\}^{f}=\frac{1}{2}\left(a e_{1} \otimes \Phi_{1}^{f}+a \Phi_{1}^{f} \otimes e_{1}-e_{1} \otimes \Phi_{1}^{f} a-\Phi_{1}^{f} \otimes e_{1} a\right) .
$$

The proof consists of checking (2.21) and (2.22) on generators, which is done in Appendix B.

\section{Applications}

\subsection{Elementary examples of fusion}

Given two double quasi-Poisson algebras $(A,\{\{-,-\}\})$ and $\left(A^{\prime},\{\{-,-\}\}^{\prime}\right)$ over $\mathbb{k}$, we can use Remark 2.13 to get a double quasi-Poisson bracket on $A \oplus A^{\prime}$ which is $B$-linear for $B=\mathbb{k} e_{1} \oplus \mathbb{k} e_{2}$ with $e_{1}=(1,0)$ and $e_{2}=(0,1)$. Using Theorem 2.14, we can get a double quasi-Poisson bracket on the fusion algebra $\left(A \oplus A^{\prime}\right)^{f}$ obtained by fusing $e_{2}$ onto $e_{1}$. By iterating this process, we can create new double quasi-Poisson algebras using the different examples given in Section 4. (The same holds for quasi-Hamiltonian algebras if we have moment maps.) Nevertheless, as far as we use differential double brackets, one could argue that this could already be done using Van den Bergh's results [20, Theorems 5.3.1,5.3.2]. Hence, we now give new examples that involve double brackets that are not differential. To do so, recall from Example 2.3 that for any $k \geq 3, \mathbb{k}[x] /\left(x^{k}\right)$ has a double bracket given by $\{\{x, x\}\}=\frac{1}{2}\left(x^{2} \otimes 1-1 \otimes x^{2}\right)$ which is not differential. The double bracket is in fact quasi-Poisson, e.g. as a consequence of Proposition 4.1.

Example 3.1 Fix $k \geq 3$ and form $A=\mathbb{k}[x] /\left(x^{k}\right)$ which is a double quasi-Poisson algebra. Let $A^{\prime}$ be an arbitrary double quasi-Poisson $\mathbb{k}$-algebra. Then we can consider $A \oplus A^{\prime}$ with idempotents $e_{1}=(1,0), e_{2}=(0,1)$. For $B=\mathbb{k} e_{1} \oplus \mathbb{k} e_{2}, A \oplus A^{\prime}$ has a $B$-linear double quasi-Poisson bracket by Remark 2.13. We can form the fusion algebra $\bar{A}=\left(A \oplus A^{\prime}\right)^{f}$ obtained by fusing $e_{2}$ onto $e_{1}$, which we see as an algebra over $\mathbb{k}$ by identifying the only remaining non-zero idempotent $e_{1}$ with 1. Using Lemma 2.11, $\bar{A}$ is the algebra generated by $x$ and $e_{12} w e_{21}$ for $w \in A^{\prime}$. Thus, we can identify $\bar{A}$ with $A *_{\mathbb{k}} A^{\prime}$, and see the elements of $A$ as generators of type 1 (2.6a) after fusion, 
while the elements of $A^{\prime}$ are generators of type 4 (2.6d). Therefore, using Theorem 2.14, we have a double quasi-Poisson bracket on $\bar{A}$ given by

$$
\{\{x, w\}\}=\frac{1}{2}(w x \otimes 1+1 \otimes x w-w \otimes x-x \otimes w), \quad w \in A^{\prime},
$$

if we use Eq. 2.14d in Lemma 2.19, while the double brackets $\{\{x, x\}\}$ and $\left\{\left\{w, w^{\prime}\right\}\right\}$ for $w, w^{\prime} \in$ $A^{\prime}$ are just the ones in $A$ and $A^{\prime}$ respectively.

Example 3.2 Fix integers $M \geq 1$ and $k_{s} \geq 3$ for $1 \leq s \leq M$. We can form $A_{s}=\mathbb{k}\left[x_{s}\right] /\left(x_{s}^{k_{s}}\right)$ and consider $A=\oplus_{s} A_{s}$ where we denote each unit by $e_{s}$ so that $A$ is an algebra over $B=\oplus_{s} \mathbb{k} e_{s}$. Moreover, it has a double quasi-Poisson bracket by Remark 2.13. Fusing $e_{2}$ onto $e_{1}$, then $e_{3}$ onto $e_{1}$ and so on up to $e_{M}$, we get the fusion algebra

$$
A^{\prime}=\mathbb{k}\left\langle x_{1}, \ldots, x_{M}\right\rangle / I, \quad \text { where } I \text { is the ideal generated by } x_{1}^{k_{1}}, \ldots, x_{M}^{k_{M}},
$$

which is just a $\mathbb{k}$-algebra. By Theorem 2.14 and Lemma $2.19, A^{\prime}$ has a double quasi-Poisson bracket given by

$$
\begin{array}{cl}
\left\{\left\{x_{s}, x_{s}\right\}\right\}=\frac{1}{2}\left(x_{s}^{2} \otimes 1-1 \otimes x_{s}^{2}\right), & 1 \leq s \leq M, \\
\left\{\left\{x_{r}, x_{s}\right\}\right\}=\frac{1}{2}\left(x_{s} x_{r} \otimes 1+1 \otimes x_{r} x_{s}-x_{s} \otimes x_{r}-x_{r} \otimes x_{s}\right), & 1 \leq r<s \leq M .
\end{array}
$$

I have been unable to find a quasi-Hamiltonian algebra whose double bracket is not differential. It is an interesting question to determine if such an example exists, in order to see whether Theorem 2.15 is strictly stronger than [20, Theorem 5.3.2] or not.

\subsection{Revisiting Van den Bergh's double bracket for quivers}

\subsubsection{Generalities}

Let $Q$ be a finite quiver with vertex set denoted $I$. We define the functions $t, h: Q \rightarrow I$ that associate to an arrow $a$ either its tail $t(a) \in I$ or its head $h(a) \in I$. We form the double $\bar{Q}$ of the quiver $Q$ with the same vertex set $I$ by adding an opposite arrow $a^{*}: h(a) \rightarrow t(a)$ to each $a \in Q$. We naturally extend $h, t$ to $\bar{Q}$, and set $\left(a^{*}\right)^{*}=a$ for each $a \in Q$ so that the map $a \mapsto a^{*}, a \in \bar{Q}$, defines an involution on $\bar{Q}$. We form the path algebra $\mathbb{k} \bar{Q}$ which is the $\mathbb{k}$-algebra generated by the arrows $a \in \bar{Q}$ and idempotents $\left(e_{s}\right)_{s \in I}$ labelled by the vertices such that

$$
a=e_{t(a)} a e_{h(a)}, \quad e_{s} e_{t}=\delta_{s t} e_{s} .
$$

This implies that we read paths from left to right. We see $\mathbb{k} \bar{Q}$ as a $B$-algebra with $B=$ $\oplus_{s \in I} \mathbb{k} e_{s}$.

We define $\epsilon: \bar{Q} \rightarrow\{ \pm 1\}$ as the map which takes value +1 on arrows originally in $Q$, and -1 on the arrows in $\bar{Q} \backslash Q$. For each $a \in Q$, we also choose $\gamma_{a} \in \mathbb{k}$ and set $\gamma_{a^{*}}=\gamma_{a}$. Finally, we associate to $\mathbb{k} \bar{Q}$ the algebra $A$ obtained by universal localisation from the set $S=\left\{1+\left(\gamma_{a}-1\right) e_{t(a)}+a a^{*} \mid a \in \bar{Q}\right\}$. This is equivalent to add local inverses $\left(\gamma_{a} e_{t(a)}+a a^{*}\right)^{-1}$ for each $a \in \bar{Q}$ (i.e. they are inverses to $\gamma_{a} e_{t(a)}+a a^{*}$ in $\left.e_{t(a)} A e_{t(a)}\right)$. If $\gamma_{a}=0$, then $a^{-1}:=$ $a^{*}\left(a a^{*}\right)^{-1}$ satisfies $a^{-1}=\left(a^{*} a\right)^{-1} a^{*}$, so that $a a^{-1}=e_{t(a)}$ and $a^{-1} a=e_{h(a)}$; the same holds for $a^{*}$.

\subsubsection{The quasi-Hamiltonian structure}

For each vertex $s \in I$, consider a total ordering $<_{s}$ on the set $T_{s}=\{a \in \bar{Q} \mid t(a)=s\}$. Write $o_{s}(-,-)$ for the ordering function at vertex $s$ : on arrows $a, b$ we have $o_{s}(a, b)=+1$ 
if $a<_{s} b, o_{s}(a, b)=-1$ if $b<_{s} a$, while it is zero otherwise, i.e. if $a=b \in T_{s}$, if $a \notin T_{s}$ or if $b \notin T_{s}$.

Theorem 3.3 The algebra A has a double quasi-Poisson bracket defined by

$$
\begin{aligned}
\{\{a, a\}\}= & \frac{1}{2} o_{t(a)}\left(a, a^{*}\right)\left(a^{2} \otimes e_{t(a)}-e_{h(a)} \otimes a^{2}\right) \quad(a \in \bar{Q}), \\
\left\{\left\{a, a^{*}\right\}\right\}= & \gamma_{a} e_{h(a)} \otimes e_{t(a)}+\frac{1}{2} a^{*} a \otimes e_{t(a)}+\frac{1}{2} e_{h(a)} \otimes a a^{*} \\
& +\frac{1}{2} o_{t(a)}\left(a, a^{*}\right)\left(a^{*} \otimes a-a \otimes a^{*}\right) \quad(a \in Q),
\end{aligned}
$$

and for $b, c \in \bar{Q}$ such that $c \neq b, b^{*}$

$$
\begin{aligned}
\{\{b, c\}\}= & -\frac{1}{2} o_{t(b)}(b, c)(b \otimes c)-\frac{1}{2} o_{h(b)}\left(b^{*}, c^{*}\right)(c \otimes b) \\
& +\frac{1}{2} o_{t(b)}\left(b, c^{*}\right) c b \otimes e_{t(b)}+\frac{1}{2} o_{h(b)}\left(b^{*}, c\right) e_{h(b)} \otimes b c .
\end{aligned}
$$

Furthermore, A is quasi-Hamiltonian for the multiplicative moment map

$$
\Phi=\sum_{s} \Phi_{s}, \quad \Phi_{s}=\prod_{a \in T_{s}}\left(\gamma_{a} e_{s}+a a^{*}\right)^{\epsilon(a)} .
$$

In Eq. 3.3, we take the product defining $\Phi_{s}$ with respect to the ordering on $T_{s}$. If all $\gamma_{a}=+1$, this result explicitly gives the double bracket defined from a poly-vector field $P \in\left(D_{B} A\right)_{2}$ in [20, Theorem 6.7.1], which was written in the above form for particular choices of ordering in [8, Proposition 2.6]. In fact, if all $\gamma_{a} \neq 0$, the result is equivalent to the previous case up to rescaling. If some $\gamma_{a}$ are equal to zero, our result also encompasses the generalisation proposed in [8, Proposition 2.7].

\subsubsection{Proof of Theorem 3.3}

As in the proof of [20, Theorem 6.7.1], we begin with the quiver $Q^{\text {sep }}$ which has vertex and arrow sets given by

$$
I^{s e p}=\left\{v_{b}, v_{b^{*}} \mid b \in Q\right\}, \quad Q^{s e p}=\left\{b: v_{b} \rightarrow v_{b^{*}} \mid b \in Q\right\} .
$$

We form the double $\bar{Q}^{s e p}$ of $Q^{s e p}$, which amounts to add the arrows $\left\{b^{*}: v_{b^{*}} \rightarrow v_{b} \mid b \in Q\right\}$. We define on it the involution $*$ given by $b \mapsto b^{*}$ and $b^{*} \mapsto b$. We add local inverses $\left(\gamma_{b} e_{v_{b}}+b b^{*}\right)^{-1}$ in $\mathbb{k} \bar{Q}^{s e p}$ for all $b \in \bar{Q}^{\text {sep }}$ to get the algebra $A^{s e p}$. By combining Example 4.6 (with $t=b, s=b^{*}$ for each $b \in Q^{s e p}$ ) and Remark 2.13, $A^{s e p}$ is quasi-Hamiltonian for the double quasi-Poisson bracket given by

$$
\left\{\left\{b, b^{*}\right\}\right\}=\gamma_{b} e_{v_{b^{*}}} \otimes e_{v_{b}}+\frac{1}{2} b^{*} b \otimes e_{v_{b}}+\frac{1}{2} e_{v_{b^{*}}} \otimes b b^{*},
$$

for all $b \in Q^{s e p}$ and which is zero on every other pair of generators, while the multiplicative moment map is defined as

$$
\Phi=\sum_{b \in \bar{Q}^{s e p}} \Phi_{v_{b}}, \quad \Phi_{v_{b}}=\left(\gamma_{b} e_{v_{b}}+b b^{*}\right)^{\epsilon(b)} .
$$

To get a quasi-Hamiltonian structure on $A$, it remains to fuse all these disjoint quivers of $\bar{Q}^{\text {sep }}$ according to the ordering that we chose at the vertices of $\bar{Q}$. More precisely, 
label the vertices in the quiver $\bar{Q}$ as $\{1, \ldots,|I|\}$, and label the arrows according to the ordering, that is if the arrow $b$ is the $k$-th element with respect to the total ordering on $T_{s}$ (going from the minimal to the maximal element in the chain) where $s=t(b)$, we label it $a_{s, k}$. We use the same names for the arrows in $\bar{Q}^{\text {sep }}$. To recover $\bar{Q}$, we rename $v_{a_{1,1}}$ as 1 , then fuse 1 and $v_{a_{1,2}}$ which we still name 1 , then continue with all vertices labelled $v_{a_{1, k}}$ for increasing values of $k$. Next, we do the same for vertices $2, \ldots,|I|$ and recover the quiver $\bar{Q}$. In terms of algebras, this means that we consider the fusion algebra obtained after fusing $e_{v_{1,2}}$ onto $e_{1}$, then $e_{v_{1,3}}$ onto $e_{1}$, and so on. This finally yields the algebra $A$. Therefore, it suffices to use Theorems 2.14 and 2.15 to get the desired result. We directly find that $\Phi$ is given by Eq. 3.3, but understanding the double bracket requires some work.

We first show Eqs. 3.1a and 3.1b, where there is nothing to prove if $a$ is not a loop. So assume that $a$ is a loop, and $a<_{t(a)} a^{*}$. By construction the only new terms arise when we glue $w_{1}:=v_{a}$ with $w_{2}:=v_{a^{*}}$, so to compute these terms we use Theorem 2.14 with the vertices $w_{1}, w_{2}$ respectively playing the role of 1,2 . We have that after fusion $a$ is a generator of third type Eq. 2.6c, so that by Eq. $2.16 \mathrm{c}$ the fusion amounts to add a term $\frac{1}{2} a^{2} \otimes e_{t(a)}-\frac{1}{2} e_{t(a)} \otimes a^{2}$ in $\{\{a, a\}\}$. Similarly, $a^{*}$ is a generator of second type Eq. $2.6 \mathrm{~b}$ so by Eq. $2.15 \mathrm{~b}$ we get a term $\frac{1}{2} e_{t(a)} \otimes\left(a^{*}\right)^{2}-\frac{1}{2}\left(a^{*}\right)^{2} \otimes e_{t(a)}$ in $\left\{\left\{a^{*}, a^{*}\right\}\right\}$. Using Eq. 2.16b, we get an additional term $\frac{1}{2} a^{*} \otimes a-\frac{1}{2} a \otimes a^{*}$ in $\left\{\left\{a, a^{*}\right\}\right\}$, which gives the correct double bracket by adding (3.5). In the case $a^{*}<_{t(a)} a$, take $w_{1}:=v_{a^{*}}$ with $w_{2}:=v_{a}$ and the proof is similar, but now $a$ is of second type and $a^{*}$ is of third type.

Before proving (3.2), we need some preparation. Consider $\alpha, \beta \in \bar{Q}$ and $s \in I$ with $\alpha<_{s} \beta, \alpha \neq \beta, \beta^{*}$. With the labelling given above, we have that $\alpha=a_{s, k_{0}}, \beta=a_{s, k_{1}}$ for some $1 \leq k_{0}<k_{1} \leq\left|T_{s}\right|$, and $v_{\alpha}=v_{a_{s, k_{0}}}, v_{\beta}=v_{a_{s, k_{1}}}$. Write $\bar{Q}^{\alpha}$ for the quiver obtained from $\bar{Q}^{s e p}$ by fusing all the vertices $v_{a_{s^{\prime}, k}}$ with either $s^{\prime}<s$, or $s^{\prime}=s$ with $k<_{s} k_{1}$ (i.e. we fuse all vertices up to excluding $v_{\beta}$ ); set $t_{\alpha}$ and $h_{\alpha}$ for the tail and head maps in $\bar{Q}^{\alpha}$. Write $\bar{Q}^{\beta}$ for the quiver obtained from $\bar{Q}^{\alpha}$ by additionally fusing the vertex $v_{a_{s, k_{1}}}$ (i.e. we fuse all vertices in $\bar{Q}^{\text {sep }}$ up to including $v_{\beta}$ ). Set again $t_{\beta}$ and $h_{\beta}$ for the associated tail and head maps. We let $A^{\alpha}$ and $A^{\beta}$ respectively denote the algebras obtained from $A^{\text {sep }}$ by fusion to arrive at the quivers $\bar{Q}^{\alpha}$ and $\bar{Q}^{\beta}$.

Lemma 3.4 The step of performing fusion from $A^{\alpha}$ to $A^{\beta}$ amounts to add the following terms in the double quasi-Poisson bracket of $A$ between the elements $\alpha, \alpha^{*}$ and $\beta, \beta^{*}$ :

$$
\begin{array}{rc}
-\frac{1}{2} \alpha \otimes \beta+\frac{1}{2} \delta_{t_{\beta}(\alpha), t_{\beta}\left(\alpha^{*}\right)} e_{t_{\beta}(\alpha)} \otimes \alpha \beta & \text { in }\{\{\alpha, \beta\}\}, \\
+\frac{1}{2} \beta^{*} \alpha \otimes e_{t_{\beta}(\alpha)}-\frac{1}{2} \delta_{t_{\beta}(\alpha), t_{\beta}\left(\alpha^{*}\right)} \beta^{*} \otimes \alpha & \text { in }\left\{\left\{\alpha, \beta^{*}\right\}\right\}, \\
+\frac{1}{2} e_{t_{\beta}(\alpha)} \otimes \alpha^{*} \beta-\frac{1}{2} \delta_{t_{\beta}(\alpha), t_{\beta}\left(\alpha^{*}\right)} \alpha^{*} \otimes \beta & \text { in }\left\{\left\{\alpha^{*}, \beta\right\}\right\}, \\
-\frac{1}{2} \beta^{*} \otimes \alpha^{*}+\frac{1}{2} \delta_{t_{\beta}(\alpha), t_{\beta}\left(\alpha^{*}\right)} \beta^{*} \alpha^{*} \otimes e_{t_{\beta}(\alpha)} & \text { in }\{\{\alpha, \beta\}\} .
\end{array}
$$

Proof We know that $h_{\alpha}(\beta) \neq t_{\alpha}(\beta)$ (otherwise it would contradict the order in which we glue vertices), so we have that $\alpha, \alpha^{*}$ are generators of the first type, $\beta$ is a generator of the second type and $\beta^{*}$ is a generator of the third type in the algebra $A^{\beta}$ obtained after fusing $w_{1}:=v_{\alpha}$ and $w_{2}:=v_{\beta}$. We have by Eq. $2.14 \mathrm{~b}$ that the following terms appear in the double quasi-Poisson bracket $\{\{-,-\}\}_{\beta}$ on $A^{\beta}$ for $\{\{\alpha, \beta\}\}_{\beta}: \frac{1}{2}\left(e_{w_{1}} \otimes \alpha \beta-\alpha \otimes \beta\right)$. The first term is non-zero only if $h_{\beta}(\alpha)=t_{\beta}(\beta)$, or $t_{\beta}\left(\alpha^{*}\right)=t_{\beta}(\alpha)$, hence we can multiply it by $\delta_{t_{\beta}(\alpha), t_{\beta}\left(\alpha^{*}\right)}$. After all fusions are performed, $w_{1}$ is just $t_{\beta}(\alpha)$ and we get Eq. 3.7a. 
Using again (2.14b) then twice (2.14c) amounts to add the terms

$$
\begin{aligned}
& \frac{1}{2}\left(e_{w_{1}} \otimes \alpha^{*} \beta-e_{w_{1}} \alpha^{*} \otimes \beta\right) \text { in }\left\{\left\{\alpha^{*}, \beta\right\}\right\}_{\beta}, \\
& \frac{1}{2}\left(\beta^{*} \alpha \otimes e_{w_{1}}-\beta^{*} \otimes \alpha e_{w_{1}}\right) \text { in }\left\{\left\{\alpha, \beta^{*}\right\}\right\}_{\beta}, \\
& \frac{1}{2}\left(\beta^{*} \alpha^{*} \otimes e_{w_{1}}-\beta^{*} \otimes \alpha^{*}\right) \text { in }\left\{\left\{\alpha^{*}, \beta^{*}\right\}\right\}_{\beta} .
\end{aligned}
$$

A discussion as in the first case allows to get Eqs. 3.7b-3.7d.

To prove (3.2), we have to show that the equality holds for any kind of ordering when the two arrows meet, as it is trivially zero if they do not. We first show what happens if they meet at exactly one vertex.

If $t(b)=t(c)$, assuming that $b<_{t(b)} c$ we get by Eq. 3.7a with $\alpha=b, \beta=c$ a term $-\frac{1}{2} b \otimes c$ in $\{\{b, c\}\}$. If instead $c<_{t(b)} b$, we get by Eq. 3.7a with $\alpha=c, \beta=b$ a term $-\frac{1}{2} c \otimes b$ in $\{\{c, b\}\}$, hence a term $+\frac{1}{2} b \otimes c$ in $\{\{b, c\}\}$ by cyclic antisymmetry. This proves (3.2) in this case.

Next, assuming only $t(b)=h(c)$ and $b<_{t(b)} c^{*}$, we get by Eq. 3.7b with $\alpha=b, \beta=c^{*}$ a term $+\frac{1}{2} c b \otimes e_{t(b)}$ in $\{\{b, c\}\}$. If $c^{*}<_{t(b)} b$, we use Eq. 3.7c with $\alpha=c^{*}, \beta=b$ to get a term $+\frac{1}{2} e_{t\left(c^{*}\right)} \otimes c b$ in $\{\{c, b\}\}$, so this gives $-\frac{1}{2} c b \otimes e_{t(b)}$ as expected.

Then, for $h(b)=t(c)$ with $b^{*}<_{h(b)} c$, we have from Eq. 3.7c with $\alpha=b^{*}, \beta=c$ the term $+\frac{1}{2} e_{t\left(b^{*}\right)} \otimes b c$ in $\{\{b, c\}\}$. If $c<_{h(b)} b^{*}$ instead, we have from Eq. 3.7b with $\alpha=c, \beta=b^{*}$ the term $+\frac{1}{2} b c \otimes e_{t(c)}$ in $\{\{c, b\}\}$, which yields $-\frac{1}{2} e_{h(b)} \otimes b c$ in $\{\{b, c\}\}$ and also finishes this case.

Finally, we assume $h(b)=h(c)$. If $b^{*}<_{h(b)} c^{*}$, we get by Eq. 3.7d with $\alpha=b^{*}, \beta=c^{*}$ the contributing term $-\frac{1}{2} c \otimes b$ in $\{\{b, c\}\}$, while for $c^{*}<_{h(b)} b^{*}$ we obtain a term $-\frac{1}{2} b \otimes c$ in $\{\{c, b\}\}$, and thus $+\frac{1}{2} c \otimes b$ in $\{\{b, c\}\}$ as desired.

If $b, c$ meet at two vertices but none of them is a loop, we can conclude by adding together the two corresponding results just derived. Hence, it remains the tedious computation to check the cases when at least $b$ or $c$ is a loop. We now write two illuminating cases where $h(b)=t(b)=t(c)$, and leave to the reader the task to verify all the remaining cases (noting that we only need to check half these cases because of the cyclic antisymmetry) using Eqs. 3.7a-3.7d.

Assume that $h(b)=t(b)=t(c)$ and $b<_{t(b)} b^{*}<_{h(b)} c$. When we first glue the vertices $v_{b}, v_{b^{*}}$ in $\bar{Q}^{s e p}$ corresponding to $t(b), h\left(b^{*}\right)$, no term contributes to $\{\{b, c\}\}$. Hence, we only need to understand what happens when we glue the vertices corresponding to $t(b)=h(b)$ and $t(c)$, and by Eq. 3.7a with $\alpha=b, \beta=c$ we get the term $-\frac{1}{2} b \otimes c+\frac{1}{2} e_{t(b)} \otimes b c$, as expected. (Alternatively, we could have used Eq. 3.7c with $\alpha=b^{*}, \beta=c$ to get the same answer. It is important to remark that we glue vertices not arrows, so that only one of these two cases has to be considered, not both together.)

Assume that $h(b)=t(b)=t(c)$ and $b<_{t(b)} c<_{h(b)} b^{*}$. When gluing the vertices of $\bar{Q}^{\text {sep }}$ corresponding to $t(b)$ and $t(c)$, we get by Eq. 3.7a with $\alpha=b, \beta=c$ the only term $-\frac{1}{2} b \otimes c$ contributing to $\{\{b, c\}\}$ since $b$ is not (yet) a loop. Next, when we glue $t(c)=t(b)$ and $h(b)$, we get by Eq. 3.7b with $\alpha=c, \beta=b^{*}$ a term $+\frac{1}{2} b c \otimes e_{t(c)}$ in $\{\{c, b\}\}$ since $c$ is not a loop, hence the term $-\frac{1}{2} e_{t(b)} \otimes b c$ contributes to $\{\{b, c\}\}$ and we are done.

\subsection{Double quasi-Poisson brackets for fundamental groups of surfaces}

Let $\Sigma$ denote a compact connected surface with fixed orientation, and such that it has a non-empty boundary $\partial \Sigma$. We denote by $g \geq 0$ its genus, and $r+1 \geq 1$ the number of boundary components. Let $* \in \partial \Sigma$ be a base point, and denote by $\pi_{1}(\Sigma, *)$ the corresponding 
fundamental group of $\Sigma$. The algebra $A=\mathbb{k} \pi_{1}(\Sigma, *)$ can be presented in terms of generators $\alpha_{i}^{ \pm 1}, \beta_{i}^{ \pm 1}, \gamma_{k}^{ \pm 1}, \Phi^{ \pm 1}, 1 \leq i \leq g, 1 \leq k \leq r$, subject to the relation

$$
\prod_{1 \leq i \leq g}\left[\alpha_{i}, \beta_{i}\right] \prod_{1 \leq k \leq r} \gamma_{k}=\Phi .
$$

Here, $\Phi$ represents the loop around the boundary component containing $*$ (with suitable orientation, see Fig. 1), and we used the multiplicative commutator $[\alpha, \beta]=\alpha \beta \alpha^{-1} \beta^{-1}$. Note that in the products we write the factors from the left to the right with increasing indices.

Our aim is to give an alternative proof relying only on fusion of the next result due to Massuyeau and Turaev [14], which endows $A$ with a quasi-Hamiltonian algebra structure. (We rescale their double bracket by a factor $1 / 2$.) Hence, this proof is the non-commutative analogue of the fusion process for representation varieties [3].

Theorem 3.5 For the presentation considered above, the algebra $A=\mathbb{k} \pi_{1}(\Sigma, *)$ has a double quasi-Poisson bracket defined for any $1 \leq i \leq g$ by

$$
\begin{aligned}
& \left\{\left\{\alpha_{i}, \alpha_{i}\right\}\right\}=\frac{1}{2}\left(\alpha_{i}^{2} \otimes 1-1 \otimes \alpha_{i}^{2}\right), \quad\left\{\left\{\beta_{i}, \beta_{i}\right\}\right\}=-\frac{1}{2}\left(\beta_{i}^{2} \otimes 1-1 \otimes \beta_{i}^{2}\right), \\
& \left\{\left\{\alpha_{i}, \beta_{i}\right\}\right\}=\frac{1}{2}\left(\beta_{i} \alpha_{i} \otimes 1+1 \otimes \alpha_{i} \beta_{i}-\alpha_{i} \otimes \beta_{i}+\beta_{i} \otimes \alpha_{i}\right),
\end{aligned}
$$

for any $\phi_{i} \in\left\{\alpha_{i}, \beta_{i}\right\}, 1 \leq i \leq g$, and $i<j$, it is defined by

$$
\left\{\left\{\phi_{i}, \phi_{j}\right\}\right\}=\frac{1}{2}\left(\phi_{j} \phi_{i} \otimes 1+1 \otimes \phi_{i} \phi_{j}-\phi_{i} \otimes \phi_{j}-\phi_{j} \otimes \phi_{i}\right),
$$

for any $\phi_{i} \in\left\{\alpha_{i}, \beta_{i}\right\}, 1 \leq i \leq g$, and $1 \leq k \leq r$, it is defined by

$$
\left\{\left\{\phi_{i}, \gamma_{k}\right\}\right\}=\frac{1}{2}\left(\gamma_{k} \phi_{i} \otimes 1+1 \otimes \phi_{i} \gamma_{k}-\phi_{i} \otimes \gamma_{k}-\gamma_{k} \otimes \phi_{i}\right),
$$

and for any $1 \leq k \leq r$ and $k<l$, it is defined by

$$
\begin{aligned}
& \left\{\left\{\gamma_{k}, \gamma_{k}\right\}\right\}=\frac{1}{2}\left(\gamma_{k}^{2} \otimes 1-1 \otimes \gamma_{k}^{2}\right), \\
& \left\{\left\{\gamma_{k}, \gamma_{l}\right\}\right\}=\frac{1}{2}\left(\gamma_{l} \gamma_{k} \otimes 1+1 \otimes \gamma_{k} \gamma_{l}-\gamma_{k} \otimes \gamma_{l}-\gamma_{l} \otimes \gamma_{k}\right) .
\end{aligned}
$$

Furthermore, for any $a=\alpha_{i}, \beta_{i}, \gamma_{k}$, the double bracket with $\Phi$ is given by

$$
\{\{\Phi, a\}\}=\frac{1}{2}(a \otimes \Phi-1 \otimes \Phi a+a \Phi \otimes 1-\Phi \otimes a) .
$$

In particular, $\Phi$ is a multiplicative moment map, and $A$ is quasi-Hamiltonian.
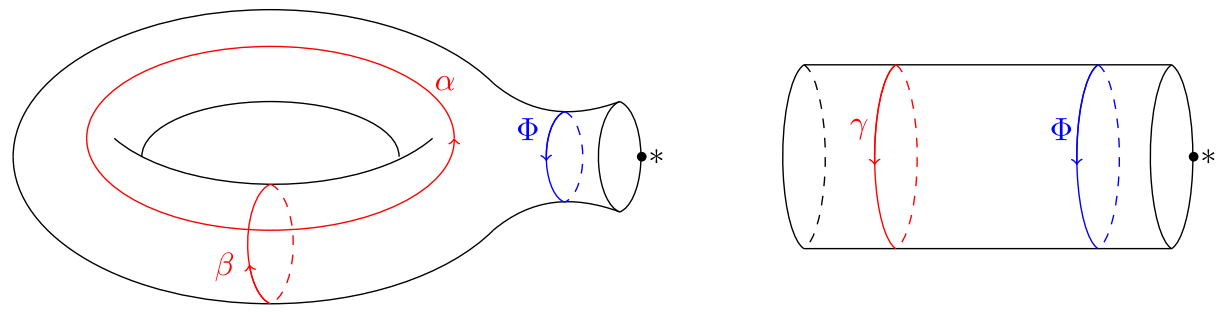

Fig. 1 A system of loops on $\Sigma$ in the cases $(g, r)=(1,0)$ and $(g, r)=(0,1)$. They can be used as generators for $\pi_{1}(\Sigma, *)$ after being connected to the base point $* \in \partial \Sigma$ in a natural way 
Proof We skip the trivial case $g=r=0$ where $A=\mathbb{k}$. If $g=0, r=1$, we have the generators of the boundary components, call them $\gamma, \Phi$, with $\Phi$ corresponding to the component containing $* \epsilon$ $\partial \Sigma$. Note that the algebra $\mathbb{k}\left[\gamma^{ \pm 1}\right]$ has a double quasi-Poisson bracket $\{\{\gamma, \gamma\}\}=\frac{1}{2}\left(\gamma^{2} \otimes 1-1 \otimes \gamma^{2}\right)$ such that $\gamma$ is a moment map as we show in $\S 4$.1. Since it is isomorphic to $A_{0}=\mathbb{k}\left\langle\gamma^{ \pm 1}, \Phi^{ \pm 1}\right\rangle /(\gamma=$ $\Phi)$, we have a quasi-Hamiltonian algebra structure on $A=A_{0}$.

If $g=1, r=0$, we have two generating cycles $\alpha, \beta$ and the generator of the boundary component $\Phi$, so that $A$ is just $A_{1}=\mathbb{k}\left\langle\alpha^{ \pm 1}, \beta^{ \pm 1}, \Phi^{ \pm 1}\right\rangle /([\alpha, \beta]=\Phi)$. But the algebra $\mathbb{k}\left\langle\alpha^{ \pm 1}, \beta^{ \pm 1}\right\rangle$ is quasi-Hamiltonian by Example 4.12 (with $t=\alpha, s=\beta, \delta=1, \gamma=0$ ), with double quasi-Poisson bracket

$$
\begin{aligned}
& \{\{\alpha, \alpha\}\}=\frac{1}{2}\left(\alpha^{2} \otimes 1-1 \otimes \alpha^{2}\right), \quad\{\{\beta, \beta\}\}=-\frac{1}{2}\left(\beta^{2} \otimes 1-1 \otimes \beta^{2}\right), \\
& \{\{\alpha, \beta\}\}=\frac{1}{2}(\beta \alpha \otimes 1+1 \otimes \alpha \beta-\alpha \otimes \beta+\beta \otimes \alpha),
\end{aligned}
$$

and moment map $\Phi=[\alpha, \beta]$. By identification, we get a quasi-Hamiltonian algebra structure on $A=A_{1}$.

We now prove the general case. We consider $g$ copies of the quasi-Hamiltonian algebra $A_{1}$ and $r$ copies of $A_{0}$, and we form $A_{1} \oplus \ldots \oplus A_{1} \oplus A_{0} \oplus \ldots \oplus A_{0}$. By Remark 2.13, this is a quasiHamiltonian algebra. We denote the element $(0, \ldots, 0,1,0, \ldots, 0)$ with 1 in $i$-th position as $e_{i}$, $1 \leq i \leq g+r$. By fusing $e_{2}$ onto $e_{1}$, then $e_{3}$ onto $e_{1}$ and so on, we get a quasi-Hamiltonian algebra structure by fusion on

$$
\mathbb{k}\left\langle\alpha_{i}^{ \pm 1}, \beta_{i}^{ \pm 1}, \Phi_{i}^{ \pm 1}, \gamma_{k}^{ \pm 1}, \bar{\Phi}_{k}^{ \pm 1} \mid 1 \leq i \leq g, 1 \leq k \leq r\right\rangle /\left(\left[\alpha_{i}, \beta_{i}\right]=\Phi_{i}, \gamma_{k}=\bar{\Phi}_{k}\right),
$$

where $\alpha_{i}, \beta_{i}, \Phi_{i}$ are the images of $\alpha, \beta, \Phi$ from the $i$-th copy of $A_{1}, 1 \leq i \leq g$, while $\gamma_{k}, \bar{\Phi}_{k}$ are the images of $\gamma, \Phi$ in the $k$-th copy of $A_{0}$. Rewriting the moment map in the algebra obtained by fusion in terms of the $\Phi_{i}, \bar{\Phi}_{k}$ using Theorem 2.15 , then removing these unnecessary elements, we can rewrite the latter algebra as

$$
\mathbb{k}\left\langle\alpha_{i}^{ \pm 1}, \beta_{i}^{ \pm 1}, \gamma_{k}^{ \pm 1}, \Phi^{ \pm 1} \mid 1 \leq i \leq g, 1 \leq k \leq r\right\rangle /\left(\prod_{1 \leq i \leq g}^{\rightarrow}\left[\alpha_{i}, \beta_{i}\right] \prod_{1 \leq k \leq r}^{\rightarrow} \gamma_{k}=\Phi\right) .
$$

This is precisely $A$. The double quasi-Poisson bracket is then easily obtained from Theorem 2.14 , Lemma 2.19, and the ones on $A_{0}, A_{1}$. For example, fix $1<j \leq g$. After the step of fusion of $e_{j}$ onto $e_{1}$, any $\phi_{i} \in\left\{\alpha_{i}, \beta_{i}\right\}$ with $1 \leq i<j$ is a generator of first type (2.6a) while $\phi_{j} \in\left\{\alpha_{j}, \beta_{j}\right\}$ is a generator of fourth type (2.6d), so that $\left\{\left\{\phi_{i}, \phi_{j}\right\}\right\}$ gets a contribution given by Eq. $2.14 \mathrm{~d}$. The fusion of $e_{k}$ onto $e_{1}$ with $k \neq j$ does not give any additional term in $\left\{\left\{\phi_{i}, \phi_{j}\right\}\right\}$, and we obtain (3.10).

Remark 3.6 To see that the double bracket from Theorem 3.5 coincides with the one of MassuyeauTuraev, note that the double brackets that do not involve the moment map are just those given in $[14, \S 8.3]$, while for the moment map they are given in $[14, \S 9.2]$. In particular, our construction is such that the moment map is the generator of the loop at the boundary component containing $* \in \partial \Sigma$.

We should also note that our proof applies to the case of a weighted surface discussed in [14, Section 10], i.e. when we fix $n_{k} \in \mathbb{N}^{\times}, 1 \leq k \leq r$, so that the generators $\alpha_{i}, \beta_{i}, \gamma_{k}$ (see Eq. 3.15) satisfy the extra constraints $\gamma_{k}^{n_{k}}=1$ for $1 \leq k \leq r$. Indeed, we can see that the ideal generated by $\gamma^{n}-1$ in $A_{0}$ is stable under the double bracket for any $n \in \mathbb{N}^{\times}$, so that we can start the proof with the algebras $\mathbb{k}\left[\gamma_{k}^{ \pm 1}\right] /\left(\gamma_{k}^{n_{k}}-1\right)$ instead of $r$ copies of $A_{0}$.

Finally, remark that the way we are gluing components is the algebraic analogue of the boundary connected sum discussed in [14, Appendix B.2].

Remark 3.7 It is an interesting problem to determine whether we can modify the definition of double quasi-Poisson bracket and keep a non-trivial fusion property as in Theorems 2.14 and 2.15. As a motivation, note that for $A=\mathbb{k} \pi_{1}(\Sigma, *)$ the double quasi-Poisson bracket given 
in Theorem 3.5 was introduced by Massuyeau-Turaev [14] by (cyclically anti-)symmetrizing an operation $A^{\times 2} \rightarrow A^{\otimes 2}$ denoted by $\{\{-,-\}\}^{\eta}$. This means that for any $a, b \in A$,

$$
\{\{a, b\}\}=\{\{a, b\}\}^{\eta}+\frac{1}{2} 1 \otimes a b+\frac{1}{2} b a \otimes 1-\frac{1}{2} a \otimes b-\frac{1}{2} b \otimes a,
$$

see $[14, \S 7.2]$ (recall that we rescale their double bracket by a factor $1 / 2$ ). Since the couple $(A,\{\{-,-\}\})$ can be obtained by fusion, it would be interesting to see if there is an analogue proof for $\left(A,\{\{-,-\}\}^{\eta}\right)$ (note that $\{\{-,-\}\}^{\eta}$ is not a double quasi-Poisson bracket as Eq. 1.1 does not hold). An explicit form similar to Theorem 3.5 of this particular operation can be found in [2, Proposition 2.14]. For other uses of the operation $\{\{-,-\}\}^{\eta}$, see $[1,2]$ and references therein.

\subsection{Morphisms of double quasi-Poisson algebras}

Fix two double quasi-Poisson algebras $(A,\{\{-,-\}\})$ and $\left(A^{\prime},\{\{-,-\}\}^{\prime}\right)$ over a $\mathbb{k}$-algebra $B$. We say that a map $\psi: A \rightarrow A^{\prime}$ is a morphism of double quasi-Poisson algebras (over $B$ ) if $\psi$ is a morphism of $B$-algebras such that for any $a, b \in A$,

$$
(\psi \otimes \psi)\{\{a, b\}\}=\{\{\psi(a), \psi(b)\}\}^{\prime} .
$$

We say that it is an isomorphism of double quasi-Poisson algebras if $\psi$ is an isomorphism of $B$-algebras, which implies that the inverse $\psi^{-1}: A^{\prime} \rightarrow A$ is also an isomorphism of double quasi-Poisson algebras. It seems natural to seek for isomorphisms between the different double quasi-Poisson algebra structures associated to quivers by Van den Bergh [20], or the slight generalisation given by Theorem 3.3. The same problem can be formulated for the double bracket of Massuyeau-Turaev [14] given in Theorem 3.5 if we change the presentation of the fundamental group by swapping factors ${ }^{1}$ in Eq. 3.8. In fact, these results easily follow from the next proposition, which is a non-commutative version of [3, Proposition 5.7].

Proposition 3.8 Assume that $(A,\{\{-,-\}\}, \Phi)$ is a quasi-Hamiltonian algebra over $B=\oplus_{s} \mathbb{k} e_{s}$. Consider the algebra $A_{1 \leftarrow 2}^{f}$ obtained by fusing $e_{2}$ onto $e_{1}$ and the algebra $A_{1 \rightarrow 2}^{f}$ obtained by fusing $e_{1}$ onto $e_{2}$, which are both quasi-Hamiltonian algebras. Then there exists an isomorphism of double quasi-Poisson algebras $A_{1 \leftarrow 2}^{f} \rightarrow A_{1 \rightarrow 2}^{f}$ which preserves moment maps.

The proof of this statement is quite tedious, so we skip it and we will provide details in further work. Let us simply mention that the isomorphisms between multiplicative preprojective algebras with different orderings, which are given in the proof of [7, Theorem 1.4], are precisely induced by this map.

\section{Elementary classification}

All our algebras are over a field $\mathbb{k}$ of characteristic 0 for convenience, but the discussion may be adapted to any integral domain (with unit) such that 2 is invertible. One could get rid of the latter localisation by rescaling the defining property (1.6) as in [14].

\subsection{Polynomial ring in one variable}

We begin by classifying all double quasi-Poisson brackets on $A=\mathbb{k}[t]$ over $B=\mathbb{k}$. Our argument is similar to the classification of Powell [18, Proposition A.1] in the case of a double

\footnotetext{
${ }^{1}$ It was pointed out by an anonymous referee that this can be obtained from [14] by invariance of the double bracket of Massuyeau-Turaev under self-homeomorphisms of the surface $\Sigma$ preserving $*$.
} 
Poisson bracket, i.e. when the associated triple bracket (1.4) identically vanishes. We define a degree on $A$ by setting $|t|=1$, to get the decomposition $A=\oplus_{k \geq 0} \mathbb{k} t^{k}$ in homogeneous components, which can clearly be extended to $A^{\otimes n}$ : an element $a_{1} \otimes \ldots \otimes a_{n}$ is homogeneous of degree $k$ if each $a_{i}$ is homogeneous in $A$ and $\sum_{i}\left|a_{i}\right|=k$.

Proposition 4.1 A has a double bracket which is quasi-Poisson if and only if it is of the form

$$
\{\{t, t\}\}=\lambda(t \otimes 1-1 \otimes t)+\mu\left(t^{2} \otimes 1-1 \otimes t^{2}\right)+v\left(t^{2} \otimes t-t \otimes t^{2}\right),
$$

for $\lambda, \mu, v \in \mathbb{k}$ with $4\left(\mu^{2}-\lambda v\right)=1$.

Proof First, we remark that the quasi-Poisson property can be rewritten from Eq. 1.6 as requiring

$$
\{\{t, t, t\}\}=\frac{1}{4}\left(1+\tau_{(123)}+\tau_{(123)}^{2}\right)\left(1 \otimes t^{2} \otimes t-1 \otimes t \otimes t^{2}\right) .
$$

Next, following [18, Proposition A.1], we split the double bracket as $\{\{-,-\}\}=$ $\sum_{k=\min }^{\max }\{\{-,-\}\}^{k}$, where $\{\{-,-\}\}^{k}$ is its homogeneous component of degree $k$, i.e. $\{\{t, t\}\}^{k} \in$ $\oplus_{l \leq k} \mathbb{k} t^{l} \otimes \mathbb{k} t^{k-l}$. We then obtain that the decomposition of the triple bracket $\{\{-,-,-\}\}$ in homogeneous components has in highest degree the triple bracket defined by $\{\{-,-\}\}^{\text {max }}$ of degree 2 max -1 . Since Eq. 4.2 is homogeneous of degree 3, we need that the triple bracket associated to $\{\{-,-\}\}^{\max }$ vanishes if $\max \geq 3$, that is we need $\{\{-,-\}\}^{\max }$ to be a double Poisson bracket. But [18, Proposition A.1] gives that such a homogeneous double Poisson bracket is non-zero only if its degree is at most 3 . Moreover, if $\max =3$, this result also yields that it is a multiple of $\{\{t, t\}\}^{3}:=t^{2} \otimes t-t \otimes t^{2}$.

We have thus obtained that $\{\{t, t\}\}$ must be of the form (4.1) for some $\lambda, \mu, v \in \mathbb{k}$. The corresponding triple bracket is easily computed (see e.g. [18, Proposition A.1]) and gives

$$
\{\{t, t, t\}\}=\left(\mu^{2}-\lambda v\right)\left(1+\tau_{(123)}+\tau_{(123)}^{2}\right)\left(1 \otimes t^{2} \otimes t-1 \otimes t \otimes t^{2}\right),
$$

so we can conclude by comparing this last expression with (4.2).

Lemma 4.2 Assume that $A=\mathbb{k}[t]$ is endowed with a double quasi-Poisson bracket in the form (4.1), and set $\bar{A}=\mathbb{k}[t]_{(t-\lambda)}$. Then $\bar{A}$ is a quasi-Hamiltonian algebra if and only if $v=0$.

Proof First, remark that when $v=0$, we have by Proposition 4.1 that $\mu=\frac{\delta}{2}$ for some $\delta \in\{ \pm 1\}$, and $\Phi=(t-\lambda)^{\delta}$ is a moment map.

For the converse, we see $\bar{A}$ as the graded algebra $\mathbb{k}\left[\bar{t}^{ \pm 1}\right]$, where $\bar{t}=t-\lambda$ has degree +1 . We also note that Eq. 4.1 is equivalent to

$$
\{\{\bar{t}, \bar{t}\}\}=\left(\lambda+2 \lambda \mu+\lambda^{2} v\right)(\bar{t} \otimes 1-1 \otimes \bar{t})+(\mu+\lambda v)\left(\bar{t}^{2} \otimes 1-1 \otimes \bar{t}^{2}\right)+v\left(\bar{t}^{2} \otimes \bar{t}-\bar{t} \otimes \bar{t}^{2}\right) .
$$

Since $\bar{A}$ is quasi-Hamiltonian, there exists an (invertible) element $\Phi$ that satisfies

$$
\{\{\Phi, \bar{t}\}\}=\frac{1}{2}(\bar{t} \otimes \Phi-1 \otimes \Phi \bar{t}+\bar{t} \Phi \otimes 1-\Phi \otimes \bar{t}),
$$

and which we can decompose as

$$
\Phi=\sum_{k_{0} \leq l \leq k_{1}} c_{l} \bar{t}^{l}, \quad c_{k_{0}}, c_{k_{1}} \in \mathbb{k}^{\times} .
$$

Then, we get by looking at Eq. 4.5 in highest degree that $c_{k_{1}}\left\{\left\{\bar{t}^{k_{1}}, \bar{t}\right\}\right\}$ is of degree at most $k_{1}+1$. But using the derivation property (1.3), this highest degree is exactly $D+k_{1}-1$, where $D$ is the maximal degree of $\{\{\bar{t}, \bar{t}\}\}$ given in Eq. 4.4. This implies that $D \leq 2$, i.e. there is no component of degree 3 in $\{\{\bar{t}, \bar{t}\}\}$. We get from Eq. 4.4 that $v=0$. 


\subsection{Algebra with two idempotents}

In the previous case, the algebra $A$ was simply a $\mathbb{k}$-algebra with no non-trivial (i.e. distinct from 0,1$)$ idempotent elements. The simplest case where such a decomposition occurs consists in taking the path algebra $\mathbb{k} Q_{1}$ of the quiver $Q_{1}$ with vertices $\{1,2\}$ and unique arrow $t: 1 \rightarrow 2$. (For conventions on quivers and path algebras, see $\$ 3.2 .1$.) We can see $\mathbb{k} Q_{1}$ as a $B$-algebra with $B=\mathbb{k} e_{1} \oplus \mathbb{k} e_{2}$, and if we assume that we have a $B$-linear double bracket on $\mathbb{k} Q_{1}$, the derivation rules yield

$$
\{\{t, t\}\}=\left\{\left\{e_{1} t e_{2}, e_{1} t e_{2}\right\}\right\}=e_{1} * e_{1}\{\{t, t\}\} e_{2} * e_{2} .
$$

Using Sweedler's notation, this implies that $\{\{t, t\}\}^{\prime}$ and $\{\{t, t\}\}^{\prime \prime}$ are of the form $\alpha t$ for some $\alpha \in \mathbb{k}$. Therefore $\{\{t, t\}\}=\alpha t \otimes t$, and the cyclic antisymmetry implies $\alpha=0$ so that $\mathbb{k} Q_{1}$ can only be endowed with the zero double bracket. At the same time, it is easy to see that $\{\{t, t, t\}\}$ given by Eq. 1.6 vanishes for $\mathbb{k} Q_{1}$, so we get the next result.

Lemma 4.3 The zero double bracket is the unique double quasi-Poisson bracket on $\mathbb{k} Q_{1}$.

As we have seen in $\$ 4.1$, the zero double bracket is not quasi-Poisson on $\mathbb{k}[t]$, and the fact that it is quasi-Poisson on $\mathbb{k} Q_{1}$ is only due to the idempotent decomposition which implies $t^{2}=0$. In fact, if we consider $\mathbb{k}[t]$ as the fusion algebra obtained by fusing $e_{1}$ and $e_{2}$ in $\mathbb{k} Q_{1}$, the zero double quasi-Poisson bracket on $\mathbb{k} Q_{1}$ yields after fusion the case $\lambda=v=0$ in Proposition 4.1.

To get non-trivial examples of $B$-linear double brackets, we consider the double quiver $\bar{Q}_{1}$ obtained by adding to $Q_{1}$ the arrow $s=t^{*}: 2 \rightarrow 1$. If we define a degree on $A$ by setting $|s|=|t|=1$ and extend it to $A \otimes A$, we can characterise the $B$-linear double quasi-Poisson brackets on $A$ that have degree at most +4 on generators. By the latter condition, we mean that $\{\{s, s\}\},\{\{t, t\}\}$ and $\{\{t, s\}\}$ (hence $\{\{s, t\}\})$ are sums of homogeneous terms of degree at most +4 .

Proposition 4.4 Any B-linear double quasi-Poisson bracket $\{\{-,-\}\}$ on $A=\mathbb{k} \bar{Q}_{1}$ which has degree at most +4 on generators must be one of the following :

Case 1: $\quad\{\{s, s\}\}=0,\{\{t, t\}\}=0$ and one of the next two conditions holds

$$
\begin{aligned}
\text { 1.a) }\{\{t, s\}\}= & \frac{\delta}{2}\left(s t \otimes e_{1}-e_{2} \otimes t s\right), \quad \delta \in\{ \pm 1\}, \\
\text { 1.b) }\{\{t, s\}\}= & \gamma e_{2} \otimes e_{1}+\phi s t \otimes t s+\alpha\left(s t \otimes e_{1}+e_{2} \otimes t s\right), \\
& \alpha, \gamma, \phi \in \mathbb{k}, \alpha^{2}=\frac{1}{4}+\gamma \phi ;
\end{aligned}
$$

Case 2: $\quad\{\{s, s\}\}=0,\{\{t, t\}\}=\lambda(t s t \otimes t-t \otimes t s t)$ for $\lambda \in \mathbb{k}^{\times}$and

$$
\{\{t, s\}\}=\frac{\delta}{2}\left(s t \otimes e_{1}-e_{2} \otimes t s\right), \quad \delta \in\{ \pm 1\} ;
$$

Case 3: $\quad\{\{t, t\}\}=0,\{\{s, s\}\}=\lambda(s t s \otimes s-s \otimes s t s)$ for $\lambda \in \mathbb{k}^{\times}$and

$$
\{\{t, s\}\}=\frac{\delta}{2}\left(s t \otimes e_{1}-e_{2} \otimes t s\right), \quad \delta \in\{ \pm 1\} .
$$

The proof is given in Appendix C.

Example 4.5 The simplest double quasi-Poisson brackets that can be obtained from Case 1 are

$$
\{\{t, t\}\}=0, \quad\{\{s, s\}\}=0, \quad\{\{t, s\}\}=\frac{\delta}{2} s t \otimes e_{1}+\frac{\delta^{\prime}}{2} e_{2} \otimes t s, \quad \delta, \delta^{\prime} \in\{ \pm 1\} .
$$


These double brackets are all obtained by fusion. Indeed, consider the quiver $Q_{1}$ with vertices $\{1,2\}$ and unique arrow $t: 1 \rightarrow 2$, and the quiver $Q_{1}^{\prime}$ with vertices $\{3,4\}$ and unique arrow $s: 4 \rightarrow 3$. Their path algebras have a double quasi-Poisson bracket which is the zero one by Lemma 4.3. Thus, the zero double bracket on the path algebra $A$ of the quiver $Q_{1} \sqcup Q_{1}^{\prime}$ is also quasi-Poisson by Remark 2.13. We can see $A$ as an algebra over $B=\oplus_{s=1}^{4} \mathbb{k} e_{s}$, where $e_{s}$ is the elementary path corresponding to the $s$-th vertex. We can glue the vertices 1 and 3 , as well as the vertices 2 and 4 . The resulting fusion algebra is just $\mathbb{k} \bar{Q}_{1}$, and we have a double quasi-Poisson bracket by Theorem 2.14 given by Eq. 4.8, where $\delta=+1$ (resp. $\delta=-1$ ) if we fuse $e_{3}$ onto $e_{1}$ (resp. $e_{1}$ onto $e_{3}$ ), and where $\delta^{\prime}=+1$ (resp. $\left.\delta^{\prime}=-1\right)$ if we fuse $e_{4}$ onto $e_{2}$ (resp. $e_{2}$ onto $e_{4}$ ).

Example 4.6 Up to localisation, we claim that the algebra $A$ with double quasi-Poisson bracket given by Case 1 with Eq. $4.7 \mathrm{~b}$ is quasi-Hamiltonian when $\gamma \phi=0$. In such a case, we set $\alpha=\frac{\delta}{2}$ for some $\delta= \pm 1$.

If $\phi=0$, consider the localisation of $A$ at $\delta \gamma+s t$ and $\delta \gamma+t s$. This is equivalent to require that the element $\delta \gamma e_{1}+t s$ is invertible in $e_{1} A e_{1}$, while $\delta \gamma e_{2}+s t$ is invertible in $e_{2} A e_{2}$. We can easily check that $\Phi_{1}=\left(\delta \gamma e_{1}+t s\right)^{\delta}$ and $\Phi_{2}=\left(\delta \gamma e_{2}+s t\right)^{-\delta}$ satisfy (1.7). Hence $\Phi=\Phi_{1}+\Phi_{2}$ is a moment map in the localised algebra.

If $\gamma=0$, we require that $t s$ (resp. st) is invertible in $e_{1} A e_{1}$ (resp. $e_{2} A e_{2}$ ) with local inverse $(t s)^{-1}$ (resp. $\left.(s t)^{-1}\right)$. We then further require that we have local inverses for $\phi e_{1}+(t s)^{-1}$ and $\phi e_{2}+(s t)^{-1}$. As a result, we can check that $\Phi=\Phi_{1}+\Phi_{2}$ is a moment map for $\Phi_{1}=\left(\delta \phi e_{1}+\right.$ $\left.(t s)^{-1}\right)^{-\delta}$ and $\Phi_{2}=\left(\delta \phi e_{2}+(s t)^{-1}\right)^{\delta}$.

When $\gamma=\phi=0$, both constructions give the same quasi-Hamiltonian algebra.

Remark 4.7 For $\phi=0$ and $\gamma=\delta=+1$ in Example 4.6, this corresponds to Van den Bergh's key example of quasi-Hamiltonian algebra associated to the double of the quiver $1 \rightarrow 2$ given in $[20, \S 6.5]$ (see Theorem 3.3).

\subsection{Free algebra on two generators}

Consider $A=\mathbb{k}\langle s, t\rangle$ with $B=\mathbb{k}$. To obtain new examples of double quasi-Poisson brackets on $A$, we assume that we have a double bracket such that

$$
\begin{aligned}
& \{\{t, t\}\}=\lambda(t \otimes 1-1 \otimes t)+\mu\left(t^{2} \otimes 1-1 \otimes t^{2}\right)+v\left(t^{2} \otimes t-t \otimes t^{2}\right), \\
& \{\{s, s\}\}=l(s \otimes 1-1 \otimes s)+m\left(s^{2} \otimes 1-1 \otimes s^{2}\right)+n\left(s^{2} \otimes s-s \otimes s^{2}\right),
\end{aligned}
$$

with coefficients in $\mathbb{k}$ that satisfy $4\left(\mu^{2}-\lambda v\right)=1$ and $4\left(m^{2}-\ln \right)=1$. Furthermore, we consider that the double bracket between $s$ and $t$ has the form

$$
\begin{aligned}
\{\{t, s\}\}= & \alpha_{0} t^{2} \otimes 1+\alpha_{0}^{\prime} 1 \otimes t^{2}+\beta_{0} s^{2} \otimes 1+\beta_{0}^{\prime} 1 \otimes s^{2}+\gamma_{0} t \otimes t+\gamma_{1} s \otimes s \\
& +\alpha_{1} t s \otimes 1+\alpha_{1}^{\prime} s t \otimes 1+\alpha_{2} t \otimes s+\alpha_{2}^{\prime} s \otimes t+\alpha_{3} 1 \otimes t s+\alpha_{3}^{\prime} 1 \otimes s t \\
& +\beta_{1} t \otimes 1+\beta_{1}^{\prime} 1 \otimes t+\beta_{2} s \otimes 1+\beta_{2}^{\prime} 1 \otimes s+\gamma 1 \otimes 1,
\end{aligned}
$$

with all coefficients in $\mathbb{k}$. In other words, if we fix a degree on $A$ by $|t|=|s|=1$ and extend it to $A \otimes A$, we assume that the double bracket $\{\{t, s\}\}$ has degree at most +2 . We wish to formulate a classification of the double quasi-Poisson brackets of the above form. To do so, introduce the conditions

$$
\begin{array}{ll}
\text { (C1) } \lambda=v=0, \mu= \pm \frac{1}{2}, & \text { (C1') } \quad \mu=0, v=\frac{-1}{4 \lambda} \in \mathbb{k}^{\times}, \\
\text {(C2) } \quad l=n=0, m= \pm \frac{1}{2}, & \text { (C2') } \quad m=0, n=\frac{-1}{4 l} \in \mathbb{k}^{\times} .
\end{array}
$$

We say that a double bracket $\{\{-,-\}\}$ on $A$ of the form (4.9a)-(4.9b) and (4.10) is reduced if it satisfies either $(\mathrm{C} 1)$ or $\left(\mathrm{C}^{\prime}\right.$ '), together with either $(\mathrm{C} 2)$ or $\left(\mathrm{C} 2{ }^{\prime}\right)$. It is not difficult to see that, up to an affine change of variables $t \mapsto t+\rho_{t}, s \mapsto s+\rho_{s}$, for suitable $\rho_{t}, \rho_{s} \in \mathbb{k}$, any 
double bracket $\{\{-,-\}\}$ on $A$ of the form (4.9a)-(4.9b) and (4.10) can be put into reduced form.

Proposition 4.8 Any double bracket $\{\{-,-\}\}$ on A of the form (4.9a)-(4.9b) and (4.10) which is quasi-Poisson is isomorphic to one of the following reduced double quasi-Poisson brackets :

Case 1: For any $\gamma_{0}, \gamma_{1} \in \mathbb{k}, \mu= \pm \frac{1}{2}, \alpha \in \mathbb{k}$ such that $\alpha^{2}=\frac{1}{4}+\gamma_{0} \gamma_{1}$,

$$
\begin{aligned}
& \{\{t, t\}\}=\mu\left(t^{2} \otimes 1-1 \otimes t^{2}\right), \quad\{\{s, s\}\}=\mu\left(s^{2} \otimes 1-1 \otimes s^{2}\right), \\
& \{\{t, s\}\}=\gamma_{0} t \otimes t+\gamma_{1} s \otimes s+\mu(s t \otimes 1-1 \otimes t s)+\alpha(t \otimes s+s \otimes t),
\end{aligned}
$$

Case 2: For any $\gamma \in \mathbb{k}, \alpha, \mu= \pm \frac{1}{2}$,

$$
\begin{aligned}
& \{\{t, t\}\}=\mu\left(t^{2} \otimes 1-1 \otimes t^{2}\right), \quad\{\{s, s\}\}=-\mu\left(s^{2} \otimes 1-1 \otimes s^{2}\right), \\
& \{\{t, s\}\}=\alpha(s t \otimes 1+1 \otimes t s)+\mu(s \otimes t-t \otimes s)+\gamma 1 \otimes 1,
\end{aligned}
$$

Case 3: For any $m, \mu= \pm \frac{1}{2}$,

$$
\begin{aligned}
& \{\{t, t\}\}=\mu\left(t^{2} \otimes 1-1 \otimes t^{2}\right), \quad\{\{s, s\}\}=m\left(s^{2} \otimes 1-1 \otimes s^{2}\right), \\
& \{\{t, s\}\}=\mu(s t \otimes 1-t \otimes s+s \otimes t-1 \otimes t s),
\end{aligned}
$$

Case 4: For any $\alpha, m, \mu= \pm \frac{1}{2}$,

$$
\begin{aligned}
& \{\{t, t\}\}=\mu\left(t^{2} \otimes 1-1 \otimes t^{2}\right), \quad\{\{s, s\}\}=m\left(s^{2} \otimes 1-1 \otimes s^{2}\right), \\
& \{\{t, s\}\}=\alpha(s t \otimes 1-t \otimes s-s \otimes t+1 \otimes t s),
\end{aligned}
$$

Case 5: For any $n \in \mathbb{k}^{\times}, \alpha, \mu= \pm \frac{1}{2}$,

$$
\begin{aligned}
& \{\{t, t\}\}=\mu\left(t^{2} \otimes 1-1 \otimes t^{2}\right), \quad\{\{s, s\}\}=\frac{-1}{4 n}(s \otimes 1-1 \otimes s)+n\left(s^{2} \otimes s-s \otimes s^{2}\right), \\
& \{\{t, s\}\}=\alpha(s t \otimes 1-t \otimes s-s \otimes t+1 \otimes t s),
\end{aligned}
$$

Case 6: For any $n \in \mathbb{k}^{\times}, \mu= \pm \frac{1}{2}$,

$$
\begin{aligned}
& \{\{t, t\}\}=\mu\left(t^{2} \otimes 1-1 \otimes t^{2}\right), \quad\{\{s, s\}\}=\frac{-1}{4 n}(s \otimes 1-1 \otimes s)+n\left(s^{2} \otimes s-s \otimes s^{2}\right), \\
& \{\{t, s\}\}=\mu(s t \otimes 1-t \otimes s+s \otimes t-1 \otimes t s),
\end{aligned}
$$

Case 7: For any $n, v \in \mathbb{k}^{\times}, \alpha= \pm \frac{1}{2}$,

$$
\begin{aligned}
& \{\{t, t\}\}=\frac{-1}{4 v}(t \otimes 1-1 \otimes t)+v\left(t^{2} \otimes t-t \otimes t^{2}\right), \\
& \{\{s, s\}\}=\frac{-1}{4 n}(s \otimes 1-1 \otimes s)+n\left(s^{2} \otimes s-s \otimes s^{2}\right), \\
& \{\{t, s\}\}=\alpha(s t \otimes 1-t \otimes s-s \otimes t+1 \otimes t s),
\end{aligned}
$$

Remark 4.9 Under the automorphism of $A$ given by $s \mapsto t, t \mapsto s$, the cases given by (4.11), (4.12), (4.14) and (4.17) are invariant; we obtain from the other cases (4.13), (4.15) and (4.16) three additional cases that do not appear in Proposition 4.8. In particular, this explains why there is no other occurrence of the case $v \neq 0$ than in Eq. 4.17.

The proof of Proposition 4.8 is quite tedious and not interesting, so we skip it until Appendix D. The idea is to realise that the two conditions

$\{\{t, t, t\}\}=\frac{1}{4}\left(1+\tau_{(123)}+\tau_{(123)}^{2}\right)\left(1 \otimes t^{2} \otimes t-1 \otimes t \otimes t^{2}\right), \quad\{\{s, s, s\}\}=\frac{1}{4}\left(1+\tau_{(123)}+\tau_{(123)}^{2}\right)\left(1 \otimes s^{2} \otimes s-1 \otimes s \otimes s^{2}\right)$, 
obtained from Eq. 1.6 are trivially satisfied by Proposition 4.1 since we require $4\left(\mu^{2}-\lambda v\right)=1$ and $4\left(m^{2}-l n\right)=1$. Using that a triple bracket is cyclically antisymmetric and is completely determined by its value on generators, it remains to check for which coefficients we have the equalities

$$
\begin{gathered}
\{\{t, t, s\}\}=\frac{1}{4}\left(s t \otimes t \otimes 1-s t \otimes 1 \otimes t-s \otimes t^{2} \otimes 1+s \otimes t \otimes t\right. \\
\left.-t \otimes t \otimes s+t \otimes 1 \otimes t s+1 \otimes t^{2} \otimes s-1 \otimes t \otimes t s\right), \\
\{\{s, s, t\}\}=\frac{1}{4}\left(t s \otimes s \otimes 1-t s \otimes 1 \otimes s-t \otimes s^{2} \otimes 1+t \otimes s \otimes s\right. \\
\left.-s \otimes s \otimes t+s \otimes 1 \otimes s t+1 \otimes s^{2} \otimes t-1 \otimes s \otimes s t\right),
\end{gathered}
$$

also obtained from Eq. 1.6.

\subsubsection{Fusion for Proposition 4.8}

We can use Theorem 2.14 to obtain the following result.

Theorem 4.10 Up to localisation, any double quasi-Poisson bracket on A of the form (4.9a)-(4.9b) and (4.10) is isomorphic to a reduced double quasi-Poisson bracket obtained by fusion.

The proof follows by combining the different examples that we give now together with Proposition 4.8.

Example 4.11 (Fusion for Case 1.) For any $\alpha, \gamma_{0}, \gamma_{1} \in \mathbb{k}$ such that $\alpha^{2}=\frac{1}{4}+\gamma_{0} \gamma_{1}$, we can consider $\mathbb{k} \bar{Q}_{1}$ with the double quasi-Poisson bracket given by Eq. 4.7b in Proposition 4.4 with $\gamma=\gamma_{1}, \phi=\gamma_{0}$. We form the algebra $A$ by locally inverting $t s=e_{1} t s e_{1}$ and $s t=e_{2} s t e_{2}$. We can introduce $\bar{s}=(t s)^{-1} t=t(s t)^{-1} \in$ $e_{1} A e_{2}$. The double quasi-Poisson bracket descends to $A$ in such a way that

$$
\{\{t, t\}\}=0=\{\{\bar{s}, \bar{s}\}\}, \quad\{\{t, \bar{s}\}\}=\gamma_{0} t \otimes t+\gamma_{1} \bar{s} \otimes \bar{s}+\alpha(t \otimes \bar{s}+\bar{s} \otimes t) .
$$

Fusing $e_{1}$ and $e_{2}$, we get the fusion algebra $A^{f}=\mathbb{k}\left\langle t^{ \pm 1}, s^{ \pm 1}\right\rangle$ with double quasi-Poisson bracket given by Eq. 4.11, where $\mu=+\frac{1}{2}$ (resp. $\mu=-\frac{1}{2}$ ) if we fuse $e_{2}$ onto $e_{1}$ (resp. $e_{1}$ onto $e_{2}$ ) by using Eq. 2.16c (resp. Eq. 2.15b).

Example 4.12 (Fusion for Case 2.) For any $\gamma \in \mathbb{k}$ and $\delta= \pm 1$, the localisation $A$ of the path algebra $\mathbb{k} \bar{Q}_{1}$ at $a=\delta \gamma+t s$ and $b=\delta \gamma+s t$ is a quasi-Hamiltonian $B$-algebra for $B=\mathbb{k} e_{1} \oplus \mathbb{k} e_{2}$ by Example 4.6 (with $\phi=0$ ). The fusion algebra $A^{f}$ obtained by fusing $e_{2}$ onto $e_{1}$ can be identified with $\mathbb{k}\langle s, t\rangle_{a, b}$. It is a quasi-Hamiltonian algebra with double quasi-Poisson bracket

$$
\begin{aligned}
& \{\{t, t\}\}=\frac{1}{2}\left(t^{2} \otimes 1-1 \otimes t^{2}\right), \quad\{\{s, s\}\}=\frac{1}{2}\left(1 \otimes s^{2}-s^{2} \otimes 1\right), \\
& \{\{t, s\}\}=\gamma 1 \otimes 1+\frac{\delta}{2}(s t \otimes 1+1 \otimes t s)+\frac{1}{2}(s \otimes t-t \otimes s),
\end{aligned}
$$

using successively (2.16c), (2.15b) and (2.16b). The moment map $\Phi=a^{\delta} b^{-\delta}$ is obtained by Theorem 2.15 . If we fuse $e_{1}$ onto $e_{2}$ instead, the factors $\frac{1}{2}$ appearing in Eq. 4.20 are replaced by $-\frac{1}{2}$ and the moment map is now $\Phi=b^{-\delta} a^{\delta}$.

Remark 4.13 After fusion, the case $\gamma=\delta=+1$ treated in Example 4.12 corresponds to Van den Bergh's quasi-Hamiltonian algebra associated to a one-loop quiver [20] (see Theorem 3.3). The case $\gamma=0$ appears after localisation on $A^{\prime}=\mathbb{k}\left\langle s^{ \pm 1}, t^{ \pm 1}\right\rangle$ in [8], and gives the quasi-Hamiltonian structure for the fundamental group of a torus with one marked boundary component [14] (see Theorem 3.5).

Example 4.14 (Fusion for Cases 3,6.) We consider the algebra $\mathbb{k}\langle s\rangle$ with double quasi-Poisson bracket (4.9b), and $\mathbb{k} Q_{1}$ for the quiver $Q_{1}$ given by $t: 1 \rightarrow 2$ endowed with the zero double quasi-Poisson bracket. Consider 
the direct sum $A=\mathbb{k} Q_{1} \oplus \mathbb{k}\langle s\rangle$, where we denote the identity of $\mathbb{k}\langle s\rangle$ as $e_{3}$. This is a double quasi-Poisson algebra by Remark 2.13 .

If we fuse $e_{3}$ onto $e_{2}$ (resp. $e_{2}$ onto $e_{3}$ ) and call it $e_{2}$, we obtain the fusion algebra $A^{\prime}$ with double quasi-Poisson bracket $(4.9 \mathrm{~b}),\{\{t, t\}\}=0$ and

$$
\{\{t, s\}\}=\alpha\left(e_{2} \otimes t s-s \otimes t\right), \quad \alpha=+\frac{1}{2}\left(\text { resp. } \alpha=-\frac{1}{2}\right) .
$$

Then, if we fuse $e_{2}$ onto $e_{1}$ (resp. $e_{1}$ onto $e_{2}$ ) which becomes the unit in the fusion algebra $A^{\prime \prime}$, we have a double quasi-Poisson bracket given by Eq. $4.9 \mathrm{~b}$ and

$$
\{\{t, t\}\}=\mu\left(t^{2} \otimes 1-1 \otimes t^{2}\right), \quad\{\{t, s\}\}=\alpha(1 \otimes t s-s \otimes t)+\mu(s t \otimes 1-t \otimes s),
$$

where $\mu=\frac{1}{2}$ (resp. $\mu=-\frac{1}{2}$ ). When $\alpha=-\mu$, we get Eq. 4.13 if $n=l=0$, or we get Eq. 4.16 if $m=0$.

Example 4.15 (Fusion for Cases 4,5,7.) We consider the algebras $\mathbb{k}\langle t\rangle$ and $\mathbb{k}\langle s\rangle$ with double quasi-Poisson brackets (4.9a)-(4.9b). Then $A=\mathbb{k}\langle t\rangle \oplus \mathbb{k}\langle s\rangle$ is a double quasi-Poisson algebra by Remark 2.13, and we denote $e_{1}=(1,0), e_{2}=(0,1)$. If we fuse $e_{2}$ onto $e_{1}$ (resp. $e_{1}$ onto $e_{2}$ ) which is the unit in the fusion algebra $A^{\prime}$, we get a double quasi-Poisson bracket given by Eqs. $4.9 \mathrm{a}-4.9 \mathrm{~b}$ and

$$
\{\{t, s\}\}=\alpha(s t \otimes 1+1 \otimes t s-s \otimes t-t \otimes s),
$$

with $\alpha=+\frac{1}{2}$ (resp. $\alpha=-\frac{1}{2}$ ). For $n=l=v=\lambda=0$ we get Eq. 4.14 , for $m=v=\lambda=0$ we get Eq. 4.15, while for $m=\mu=0$ we get Eq. 4.17 .

\section{Representations spaces and (quasi-)Poisson algebras}

\subsection{Generalities on representation spaces}

We assume that $A$ is a finitely generated associative algebra over $B=\oplus_{s=1}^{K} \mathbb{k} e_{s}$, with $e_{s} e_{t}=$ $\delta_{s t} e_{s}$. Following [20, Section 7] (see also [6, Section 4] and [14, Section 3]), let $I=\{1, \ldots, K\}$ and choose a dimension vector $\alpha \in \mathbb{N}^{I}$, setting $N=\sum_{s \in I} \alpha_{s}$. We consider the representation space (relative to $B$ ) $\operatorname{Rep}(A, \alpha)$. The representation space is the affine scheme whose coordinate ring $\mathcal{O}(\operatorname{Rep}(A, \alpha))$ is generated by symbols $a_{i j}$ for $a \in A, 1 \leq i, j \leq N$, which satisfy

$$
(a+b)_{i j}=a_{i j}+b_{i j}, \quad(a b)_{i j}=\sum_{k=1}^{N} a_{i k} b_{k j},
$$

together with the condition that for any $1 \leq s \leq K$ the matrix $\mathcal{X}\left(e_{s}\right)=\left(\left(e_{s}\right)_{i j}\right)_{i j}$ is the $s$-th diagonal identity block of size $\alpha_{s}$. In other words, we have that $\left(e_{s}\right)_{i j}=\delta_{i j}$ if $\alpha_{1}+\ldots+\alpha_{s-1}+1 \leq$ $i, j \leq \alpha_{1}+\ldots+\alpha_{s}$, while it is zero otherwise. Note that this implies $1_{i j}=\delta_{i j}$ for all $1 \leq i, j \leq N$. To ease notations, denote by $R=\mathcal{O}(\operatorname{Rep}(A, \alpha))$ the coordinate ring, and for any $a \in A$ set $\mathcal{X}(a)$ to denote the matrix with entries $a_{i j} \in R$.

By definition of $\operatorname{Rep}(A, \alpha)$, any element $a \in A$ induces functions $\left(a_{i j}\right)_{i j}$ on $\operatorname{Rep}(A, \alpha)$, and we would like to extend this definition to derivations. We associate to any $\delta \in D_{A / B}$ the vector fields $\delta_{i j} \in \operatorname{Der}(R), 1 \leq i, j \leq N$, defined by

$$
\delta_{i j}\left(b_{k l}\right)=\delta(b)_{k j}^{\prime} \delta(b)_{i l}^{\prime \prime},
$$

and introduce the vector field-valued matrix $\mathcal{X}(\delta)$ with $(i, j)$ entry $\delta_{i j}$. We call the particular disposition of indices in Eq. 5.1 the standard index notation as in [21]. More generally, for an element $\delta=\delta_{1} \ldots \delta_{n} \in\left(D_{B} A\right)_{n}$ we define $\delta_{i j} \in \bigwedge_{R}^{n} \operatorname{Der}(R)$ from the matrix identity $\mathcal{X}(\delta)=$ $\mathcal{X}\left(\delta_{1}\right) \ldots \mathcal{X}\left(\delta_{n}\right)$, and we set tr $\mathcal{X}(\delta)=\sum_{i} \delta_{i i}$. 
Proposition 5.1 ([20, Propositions 7.5.1,7.5.2]) Assume that $\{\{-,-\}\}$ is a B-linear double bracket defined on $A$. Then there is a unique antisymmetric biderivation $\{-,-\}$ on $R$ such that

$$
\left\{a_{i j}, b_{k l}\right\}=\{\{a, b\}\}_{k j}^{\prime}\{\{a, b\}\}_{i l}^{\prime \prime},
$$

for any $a, b \in A$. Moreover, for any $a, b, c \in A$,

$$
\operatorname{Jac}\left(a_{i j}, b_{k l}, c_{u v}\right)=\{\{a, b, c\}\}_{u j, i l, k v}-\{\{a, c, b\}\}_{k j, i v, u l},
$$

where, on the left-hand side, Jac $: R^{\times 3} \rightarrow R$ is defined by

$$
\operatorname{Jac}\left(g_{1}, g_{2}, g_{3}\right)=\left\{g_{1},\left\{g_{2}, g_{3}\right\}\right\}+\left\{g_{2},\left\{g_{3}, g_{1}\right\}\right\}+\left\{g_{3},\left\{g_{1}, g_{2}\right\}\right\}, \quad g_{1}, g_{2}, g_{3} \in R,
$$

while on the right-hand side $\{\{-,-,-\}\}$ is the triple bracket $(1.4)$ defined by $\{\{-,-\}\}$, and we write for $a=a^{\prime} \otimes a^{\prime \prime} \otimes a^{\prime \prime \prime} \in A^{\otimes 3}$ that $a_{i j, k l, u v}=a_{i j}^{\prime} a_{k l}^{\prime \prime} a_{u v}^{\prime \prime \prime}$.

We now remark the following result, which will be important in $\$ 5.2$.

Lemma 5.2 Assume that $Q \in\left(D_{B} A\right)_{n}$, and denote by $\{\{-, \ldots,-\}\}$ the corresponding differential $n$-bracket given by Proposition 2.1. For any $a=a^{1} \otimes \ldots \otimes a^{n} \in A^{\otimes n}$, introduce

$$
a_{\left(u_{1} v_{1}, \ldots, u_{n} v_{n}\right)}=a_{u_{1} v_{1}}^{1} \ldots a_{u_{n} v_{n}}^{n} \in R,
$$

with indices in the set $\{1, \ldots, N\}$. Consider the natural action of $S_{n}$ on $\{1, \ldots, n\}$ and the action of $S_{n-1}$ on $\{2, \ldots, n\}$ obtained by fixing the element 1 . Then the following holds

$$
\operatorname{tr} \mathcal{X}(Q)\left(a_{u_{1} v_{1}}^{1}, \ldots, a_{u_{n} v_{n}}^{n}\right)=\sum_{\tilde{\sigma} \in S_{n-1}} \epsilon(\tilde{\sigma})\left\{\left\{a^{1}, a^{\tilde{\sigma}(2)}, \ldots, a^{\tilde{\sigma}(n)}\right\}\right\}_{\tilde{\sigma}(u, v)}
$$

where $\tilde{\sigma}(u, v):=\left(u_{\tilde{\sigma}(n)} v_{1}, u_{1} v_{\tilde{\sigma}(2)}, \ldots, u_{\tilde{\sigma}(n-1)} v_{\tilde{\sigma}(n)}\right)$, while $\epsilon(\tilde{\sigma})=+1$ if $\tilde{\sigma}$ is an even permutation, and $\epsilon(\tilde{\sigma})=-1$ if $\tilde{\sigma}$ is an odd permutation.

Proof By linearity, we can just assume that $Q=\delta^{1} \ldots \delta^{n}$ with each $\delta^{i} \in D_{A / B}$. We can write

$$
\begin{aligned}
& \operatorname{tr} \mathcal{X}(Q)\left(a_{u_{1} v_{1}}^{1}, \ldots, a_{u_{n} v_{n}}^{n}\right)=\sum_{i_{1}, \ldots, i_{n}}\left(\delta_{i_{1} i_{2}}^{1} \wedge \ldots \wedge \delta_{i_{n} i_{1}}^{n}\right)\left(a_{u_{1} v_{1}}^{1}, \ldots, a_{u_{n} v_{n}}^{n}\right) \\
= & \sum_{i_{1}, \ldots, i_{n}} \sum_{\sigma \in S_{n}} \epsilon(\sigma) \delta_{i_{1} i_{2}}^{1}\left(a_{u_{\sigma(1)} v_{\sigma(1)}}^{\sigma(1)}\right) \ldots \delta_{i_{q} i_{q+1}}^{q}\left(a_{u_{\sigma(q)} v_{\sigma(q)}}^{\sigma(q)}\right) \ldots \delta_{i_{n} i_{1}}^{n}\left(a_{u_{\sigma(n)} v_{\sigma(n)}}^{\sigma(n)}\right) .
\end{aligned}
$$

Using Eq. 5.1 and summing over all $i_{q}$, we get that this equals

$$
\begin{aligned}
& \sum_{\sigma \in S_{n}} \epsilon(\sigma)\left(\delta^{1}\left(a^{\sigma(1)}\right)^{\prime} \delta^{2}\left(a^{\sigma(2)}\right)^{\prime \prime}\right)_{u_{\sigma(1)} v_{\sigma(2)}} \ldots\left(\delta^{q}\left(a^{\sigma(q)}\right)^{\prime} \delta^{q+1}\left(a^{\sigma(q+1)}\right)^{\prime \prime}\right)_{u_{\sigma(q)} v_{\sigma(q+1)}} \ldots\left(\delta^{n}\left(a^{\sigma(n)}\right)^{\prime} \delta^{1}\left(a^{\sigma(1)}\right)^{\prime \prime}\right)_{u_{\sigma(n)} v_{\sigma(1)}} \\
& =\sum_{\sigma \in S_{n}} \epsilon(\sigma)\left(\delta^{n}\left(a^{\sigma(n)}\right)^{\prime} \delta^{1}\left(a^{\sigma(1)}\right)^{\prime \prime} \otimes \delta^{1}\left(a^{\sigma(1)}\right)^{\prime} \delta^{2}\left(a^{\sigma(2)}\right)^{\prime \prime} \otimes \ldots \otimes \delta^{n-1}\left(a^{\sigma(n-1)}\right)^{\prime} \delta^{1}\left(a^{\sigma(1)}\right)^{\prime \prime}\right)_{\sigma(u, v)}
\end{aligned}
$$

where $\sigma(u, v)=\left(u_{\sigma(n)} v_{\sigma(1)}, u_{\sigma(1)} v_{\sigma(2)}, \ldots, u_{\sigma(n-1)} v_{\sigma(n)}\right)$.

Next, remark that we can identify any $\sigma \in S_{n}$ with $\tilde{\sigma} \tau^{i}$, where $\tau=(1 \ldots n), i \in\{0, \ldots, n-1\}$, and $\tilde{\sigma} \in S_{n-1}$ acts on $\{2, \ldots, n\}$. Given $\sigma$, the pair $(i, \tilde{\sigma})$ is unique and satisfies $\epsilon(\sigma)=(n-1) i+\epsilon(\tilde{\sigma})$. Moreover, the action of $\sigma \in S_{n}$ on $A^{\otimes n}$ decomposes into the permutation $\tau^{i}$ of the factors and the action of $\tilde{\sigma} \in S_{n-1}$ fixing the first copy in the tensor product. Therefore, we can write $\operatorname{tr} \mathcal{X}(Q)\left(a_{u_{1} v_{1}}^{1}, \ldots, a_{u_{n} v_{n}}^{n}\right)$ as follows

$$
\sum_{\tilde{\sigma} \in S_{n-1}} \epsilon(\tilde{\sigma}) \sum_{i=0}^{n-1}(-1)^{(n-1) i}\left(\delta^{n}\left(a^{\sigma(n)}\right)^{\prime} \delta^{1}\left(a^{\sigma(1)}\right)^{\prime \prime} \otimes \ldots \otimes \delta^{n-1}\left(a^{\sigma(n-1)}\right)^{\prime} \delta^{1}\left(a^{\sigma(1)}\right)^{\prime \prime}\right)_{\sigma(u, v)},
$$

where $\sigma(u, v)=\left(u_{\sigma(n)} v_{\sigma(1)}, u_{\sigma(1)} v_{\sigma(2)}, \ldots, u_{\sigma(n-1)} v_{\sigma(n)}\right)$ and we put $\sigma=\tilde{\sigma} \tau^{i}$. 
Meanwhile, remark that we can get from Proposition 2.1

$$
\begin{gathered}
\left\{\left\{b^{1}, \ldots, b^{n}\right\}\right\}=\sum_{i=0}^{n-1}(-1)^{(n-1) i} \delta^{\tau^{-i}(n)}\left(b^{n}\right)^{\prime} \delta^{\tau^{-i}(1)}\left(b^{1}\right)^{\prime \prime} \otimes \ldots \otimes \delta^{\tau^{-i}(q)}\left(b^{q}\right)^{\prime} \delta^{\tau^{-i}(q+1)}\left(b^{q+1}\right)^{\prime \prime} \otimes \ldots \\
\ldots \otimes \delta^{\tau^{-i}(n-1)}\left(b^{n-1}\right)^{\prime} \delta^{\tau^{-i}(n)}\left(b^{n}\right)^{\prime \prime}
\end{gathered}
$$

If we extend the action of $S_{n-1}$ on $\{2, \ldots, n\}$ to $\{1, \ldots, n\}$ by setting $\tilde{\sigma}(1)=1$, we find that

$$
\begin{aligned}
& \sum_{\tilde{\sigma} \in S_{n-1}} \epsilon(\tilde{\sigma})\left\{\left\{a^{\tilde{\sigma}(1)}, a^{\tilde{\sigma}(2)}, \ldots, a^{\tilde{\sigma}(n)}\right\}\right\}_{\tilde{\sigma}(u, v)} \\
= & \sum_{\tilde{\sigma} \in S_{n-1}} \epsilon(\tilde{\sigma}) \sum_{i=0}^{n-1}(-1)^{(n-1) i}\left(\delta^{\tau^{-i}(n)}\left(a^{\tilde{\sigma}(n)}\right)^{\prime} \delta^{\tau^{-i}(1)}\left(a^{\tilde{\sigma}(1)}\right)^{\prime \prime} \otimes \ldots \otimes \delta^{\tau^{-i}(n-1)}\left(a^{\tilde{\sigma}(n-1)}\right)^{\prime} \delta^{\tau^{-i}(n)}\left(a^{\tilde{\sigma}(n)}\right)^{\prime \prime}\right)_{\tilde{\sigma}(u, v)} .
\end{aligned}
$$

where $\tilde{\sigma}(u, v):=\left(u_{\tilde{\sigma}(n)} v_{\tilde{\sigma}(1)}, u_{\tilde{\sigma}(1)} v_{\tilde{\sigma}(2)}, \ldots, u_{\tilde{\sigma}(n-1)} v_{\tilde{\sigma}(n)}\right)$. Now, we remark that if we simultaneously apply $\tau^{i}$ on the tensor product and on the indices $\tilde{\sigma}(u, v)$, then each term on the right-hand side is unchanged. But doing so is equivalent to replace any element $q \in\{1, \ldots, n\}$ (before applying $\tilde{\sigma}$ !) by $\tau^{i}(q)$ in the indices occurring in the tensor product as well as in $\tilde{\sigma}(u, v)$. This gives nothing else than Eq. 5.5.

We will particularly be interested in the case $n=3$, which takes the following form.

Lemma 5.3 Assume that $Q \in\left(D_{B} A\right)_{3}$, and denote by $\{\{-,-,-\}\}_{Q}$ the corresponding differential triple bracket. With the notation introduced in Lemma 5.2, we have for any $a, b, c \in A$

$$
\operatorname{tr} \mathcal{X}(Q)\left(a_{i j}, b_{k l}, c_{u v}\right)=\left(\{\{a, b, c\}\}_{Q}\right)_{u j, i l, k v}-\left(\{\{a, c, b\}\}_{Q}\right)_{k j, i v, u l} .
$$

Remark 5.4 Let us look again at Proposition 5.1 when $\{\{-,-\}\}$ is differential for some $P \in\left(D_{B} A\right)_{2}$. First, looking at Lemma 5.2 with $n=2$, the right-hand side of Eq. 5.4 is the same as the right-hand side of Eq. 5.2 when $a_{i j}=a_{u_{1} v_{1}}^{1}, b_{k l}=a_{u_{2} v_{2}}^{2}$. Hence, $\{-,-\}$ is equivalently defined by the bivector field $\operatorname{tr} \mathcal{X}(P)$ on $\operatorname{Rep}(A, \alpha)$, as first observed in [20,§7.8].

Next, note that the left-hand side of Eq. 5.3 is obtained by applying the trivector $\frac{1}{2}[\operatorname{tr} \mathcal{X}(P), \operatorname{tr} \mathcal{X}(P)]$, where $[-,-]$ is the (geometric) Schouten-Nijenhuis bracket. But it was remarked in [20, §7.7] that taking traces defines a Lie algebra homomorphism from the algebraic to the geometric Schouten-Nijenhuis bracket, so that $\operatorname{tr} \mathcal{X}\left(\{P, P\}_{\mathrm{SN}}\right)=[\operatorname{tr} \mathcal{X}(P), \operatorname{tr} \mathcal{X}(P)]$. Now, by Proposition 2.4, the triple bracket $\{\{-,-,-\}\}$ defined by $\{\{-,-\}\}$ is differential with trivector $\frac{1}{2}\{P, P\}_{\mathrm{SN}}$. Therefore, Eq. 5.3 becomes a corollary of Eq. 5.6 with $Q=\frac{1}{2}\{P, P\}_{\mathrm{SN}}$.

\subsection{Quasi-Poisson algebras}

Let $\mathfrak{g}$ be a Lie algebra over $\mathbb{k}$ such that $\mathfrak{g}$ is equipped with a non-degenerate symmetric bilinear form denoted $(-\mid-)$. Furthermore, assume that the form is $\mathfrak{g}$-invariant, i.e. $\left(\eta_{1} \mid\left[\eta_{2}, \eta_{3}\right]\right)=\left(\left[\eta_{1}, \eta_{2}\right] \mid \eta_{3}\right)$ for all $\eta_{1}, \eta_{2}, \eta_{3} \in \mathfrak{g}$. If we take dual bases $\left(\varepsilon_{i}\right),\left(\varepsilon^{i}\right)$ under $(-\mid-)$, then we can define the Cartan trivector $\phi \in \wedge^{3} \mathfrak{g}$ given by

$$
\phi=\frac{1}{12} \sum_{i, j, k}\left(\varepsilon^{i} \mid\left[\varepsilon^{j}, \varepsilon^{k}\right]\right) \varepsilon_{i} \wedge \varepsilon_{j} \wedge \varepsilon_{k} .
$$

Following [14, Section 2] from now on, we assume that $\mathfrak{g}$ acts on a commutative $\mathbb{k}$-algebra $R$ by derivation, so that the map $\mathfrak{g} \rightarrow \operatorname{Der}(R)$ is a Lie algebra homomorphism. Denoting by $\eta_{R}$ the action of $\eta \in \mathfrak{g}$ on $R$, the latter means that $\left[\eta^{1}, \eta^{2}\right]_{R}(a)=\eta_{R}^{1}\left(\eta_{R}^{2}(a)\right)-\eta_{R}^{2}\left(\eta_{R}^{1}(a)\right)$ for any $a \in R, \eta_{1}, \eta_{2} \in \mathfrak{g}$. We say that $R$ is a quasi-Poisson algebra over $\mathfrak{g}$ if $R$ is equipped with an anti-symmetric biderivation $\{-,-\}$ such that for any $\eta \in \mathfrak{g}$ and $a, b, c \in R$

$$
\begin{aligned}
& \eta_{R}(\{a, b\})=\left\{\eta_{R}(a), b\right\}+\left\{a, \eta_{R}(b)\right\}, \\
& \{a,\{b, c\}\}+\{b,\{c, a\}\}+\{c,\{a, b\}\}=\frac{1}{2} \phi_{R}(a, b, c) .
\end{aligned}
$$


Here, $\phi_{R}$ is the image of the Cartan trivector induced by the map $\mathfrak{g}^{\otimes 3} \times R^{\times 3} \rightarrow \mathbb{k}$ given by

$$
\left(\eta^{1} \otimes \eta^{2} \otimes \eta^{3}, a, b, c\right) \mapsto\left(\eta^{1} \otimes \eta^{2} \otimes \eta^{3}\right)_{R}(a, b, c):=\eta_{R}^{1}(a) \eta_{R}^{2}(b) \eta_{R}^{3}(c) .
$$

The operation $\{-,-\}$ is called a quasi-Poisson bracket. Note that if $R^{\mathfrak{g}} \subset R$ is the subalgebra of $\mathfrak{g}$-invariant elements, i.e. $R^{\mathfrak{g}}=\left\{a \in R \mid \eta_{R}(a)=0 \forall \eta \in \mathfrak{g}\right\}$, then $\{-,-\}$ descends to a Poisson bracket on $R^{\mathfrak{g}}$ since the right-hand side of Eq. $5.8 \mathrm{~b}$ vanishes.

Remark 5.5 In this work, we restrict the definition of quasi-Poisson algebra to the case where $\phi$ is the Cartan trivector (5.7), in analogy with [3,20]. Working in greater generalities, Massuyeau and Turaev considered an arbitrary element $\phi \in \bigwedge^{3} \mathfrak{g}$, from which we still get a Poisson bracket on $R^{\mathfrak{g}}[14, \S 2.2]$. This notion also encompasses Poisson algebras when we take $\mathfrak{g}=\{0\}$.

Assume that we are also given an arbitrary group $G$ acting on the left on $\mathfrak{g}$ by Lie algebra automorphisms. (We do not require that $\mathfrak{g}=\operatorname{Lie}(G)$.) For any $g \in G$, we write the action as $\eta \mapsto{ }^{g} \eta, \eta \in \mathfrak{g}$. We say that $R$ is a $(G, \mathfrak{g})$-algebra if $R$ is a $\mathfrak{g}$-algebra endowed with a compatible left $G$-action :

$$
\left({ }^{g} \eta\right)_{R} a=g \cdot \eta_{R}\left(g^{-1} . a\right), \quad g \in G, \eta \in \mathfrak{g}, a \in R .
$$

We say that $R$ is a quasi-Poisson algebra over the pair $(G, \mathfrak{g})$ if $R$ is a $(G, \mathfrak{g})$-algebra and if $R$ is a quasi-Poisson algebra over $\mathfrak{g}$ such that for any $g \in G, a, b \in R$

$$
\begin{aligned}
& g .\{a, b\}=\{g . a, g . b\}, \\
& { }^{g} \phi=\frac{1}{12} \sum_{i, j, k}\left(\varepsilon^{i} \mid\left[\varepsilon^{j}, \varepsilon^{k}\right]\right){ }^{{ }^{g} \varepsilon_{i} \wedge}{ }^{g} \varepsilon_{j} \wedge{ }^{g} \varepsilon_{k}=\phi .
\end{aligned}
$$

We easily see that if $R^{G} \subset R$ is the subalgebra of $G$-invariant elements, then the quasiPoisson bracket descends to a Poisson bracket on $R^{G} \cap R^{\mathfrak{g}}$.

We now consider $R=\mathcal{O}(\operatorname{Rep}(A, \alpha))$ as in $\$ 5.1$. The algebra $R$ is naturally endowed with an action of $\mathrm{GL}_{\alpha}=\prod_{s=1}^{K} \mathrm{GL}_{\alpha_{s}}(\mathbb{k})$, which is given in matrix notation by $g . \mathcal{X}(a)=g^{-1} \mathcal{X}(a) g$ for all $a \in A, g \in \mathrm{GL}_{\alpha}$. We can also consider the Lie algebra $\mathfrak{g}_{\alpha}=\prod_{s=1}^{K} \mathfrak{g l}_{\alpha_{s}}(\mathbb{k})$ of $\mathrm{GL}_{\alpha}$, which acts by derivation on $R$ as $\eta_{R}(\mathcal{X}(a))=[\mathcal{X}(a), \eta]$, for all $a \in A, \eta \in \mathfrak{g}_{\alpha}$. We can endow $\mathfrak{g}_{\alpha}$ with the trace pairing $\left(\eta_{1} \mid \eta_{2}\right)=\operatorname{tr}\left(\eta_{1} \eta_{2}\right)$, and consider the left adjoint action of $\mathrm{GL}_{\alpha}$ on $\mathfrak{g}_{\alpha}$ so that Eq. 5.9 is satisfied. The following result generalises [20, Theorem 7.12.2], see also [14, Lemma 4.4]. (This was already noticed by Van den Bergh without a proof, as mentioned in [20, Remark 7.12.3].)

Theorem 5.6 Assume that $(A,\{\{-,-\}\})$ is a double quasi-Poisson algebra over $B$. Then the algebra $R=$ $\mathcal{O}(\operatorname{Rep}(A, \alpha))$ is a quasi-Poisson algebra over the pair $\left(\mathrm{GL}_{\alpha}, \mathfrak{g}_{\alpha}\right)$ for the quasi-Poisson bracket defined by Proposition 5.1.

Proof Showing Eqs. 5.8a, 5.10a and 5.10b is easy, so we are left to show Eq. 5.8b on generators of the coordinate ring $R$. Hence, fix $a, b, c \in A$. We remark that by [20, Proposition 7.12.1] the 3-vector field $\phi_{R}$ is given by $\frac{1}{6} \sum_{s=1}^{K} \operatorname{tr} \mathcal{X}\left(E_{s}^{3}\right)$, hence we can write for any $1 \leq i, j, k, l, u, v \leq N$

$$
\frac{1}{2} \phi_{R}\left(a_{i j}, b_{k l}, c_{u v}\right)=\frac{1}{12} \sum_{s} \operatorname{tr} \mathcal{X}\left(E_{s}^{3}\right)\left(a_{i j}, b_{k l}, c_{u v}\right) .
$$

Using Lemma 5.3, this is the same as

$$
\frac{1}{2} \phi_{R}\left(a_{i j}, b_{k l}, c_{u v}\right)=\left(\{\{a, b, c\}\}_{\frac{1}{12} \sum_{s} E_{s}^{3}}\right)_{u j, i l, k v}-\left(\{\{a, c, b\}\}_{\frac{1}{12} \sum_{s} E_{s}^{3}}\right)_{k j, i v, u l} .
$$

But then, since the double bracket is quasi-Poisson we get by definition

$$
\frac{1}{2} \phi_{R}\left(a_{i j}, b_{k l}, c_{u v}\right)=\{\{a, b, c\}\}_{u j, i l, k v}-\{\{a, c, b\}\}_{k j, i v, u l},
$$


where the triple bracket is defined from $\{\{-,-\}\}$ using Eq. 1.4. The right-hand side of Eq. 5.11 is nothing else than $\operatorname{Jac}\left(a_{i j}, b_{k l}, c_{u v}\right)$ by Eq. 5.3.

If $\mathbb{k}$ is algebraically closed, we can use Le Bruyn-Procesi Theorem [13, Theorem 1] to get that $A^{\mathrm{GL}_{\alpha}}$ is generated by functions $\operatorname{tr} \mathcal{X}(a), a \in A$, see e.g. [6, Remark 4.3]. In particular, $A^{\mathrm{GL}_{\alpha}}=A^{\mathrm{GL}_{\alpha}} \cap A^{\mathfrak{g}_{\alpha}}$.

Corollary 5.7 Assume that $(A,\{\{-,-\}\})$ is a double quasi-Poisson algebra over $B$. If $\mathbb{k}$ is an algebraically closed field of characteristic 0 , then the algebra $R^{\mathrm{GL}_{\alpha}}=\mathcal{O}\left(\operatorname{Rep}(A, \alpha) / / \mathrm{GL}_{\alpha}\right)$ is a Poisson algebra whose Poisson bracket is induced by the quasi-Poisson bracket on $R$.

Example 5.8 Fix integers $M \geq 1$ and $k_{m} \geq 3$ for $1 \leq m \leq M$. Let $N=\max \left(k_{1}, \ldots, k_{M}\right)$. Combining Example 3.2 and Theorem 5.6, we get that the algebra

$$
R=\mathbb{k}\left[X_{m, i j} \mid 1 \leq m \leq M, 1 \leq i, j \leq N\right] /\left(X_{m}^{k_{m}}=0_{N} \text { for } X_{m}=\left(X_{m, i j}\right), 1 \leq m \leq M\right)
$$

is a quasi-Poisson algebra over the pair $\left(\mathrm{GL}_{N}(\mathbb{k}), \mathfrak{g l}_{N}(\mathbb{k})\right)$ with quasi-Poisson bracket

$$
\begin{aligned}
\left\{X_{m, i j}, X_{m, k l}\right\}=\frac{1}{2}\left(X_{m}^{2}\right)_{k j} \delta_{i l}-\frac{1}{2} \delta_{k j}\left(X_{m}^{2}\right)_{i l}, & 1 \leq m \leq M, \\
\left\{X_{m, i j}, X_{n, k l}\right\}=\frac{1}{2}\left(X_{n} X_{m}\right)_{k j} \delta_{i l}+\frac{1}{2} \delta_{k j}\left(X_{m} X_{n}\right)_{i l}-\frac{1}{2} X_{n, k j} X_{m, i l}-\frac{1}{2} X_{m, k j} X_{n, i l}, & 1 \leq m<n \leq M .
\end{aligned}
$$

When all the $\left(k_{m}\right)_{m}$ are equal, this gives a quasi-Poisson algebra structure on the coordinate ring corresponding to $M$ copies of the space of nilpotent $N \times N$ matrices.

Example 5.9 If $\mathbb{k}=\mathbb{R}$, we have by [14, Appendix B] that the double quasi-Poisson bracket of Massuyeau and Turaev given in Theorem 3.5 endows $\operatorname{Rep}\left(\mathbb{k} \pi_{1}(\Sigma, *), N\right)$ with the quasi-Poisson bracket given in [3].

\subsection{Moment maps and Poisson algebra}

Consider the quasi-Poisson algebra $(R,\{-,-\})$ over the pair $\left(\mathrm{GL}_{\alpha}, \mathfrak{g}_{\alpha}\right)$ obtained from the double quasi-Poisson algebra $(A,\{\{-,-\}\})$ by Theorem 5.6. We now assume that $A$ is a quasi-Hamiltonian algebra, i.e. it is endowed with a moment map $\Phi \in \oplus_{s} e_{s} A e_{s}$. For any $\left(q_{s}\right) \in\left(\mathbb{k}^{\times}\right)^{K}$, let $q=\sum_{s} q_{s} e_{s} \in B^{\times}$and define the ideal $J_{q}$ generated by the entries of the matrix identity $\mathcal{X}(\Phi)-\mathcal{X}(q)=0_{N}$. We can form the algebra $R_{q}=R / J_{q}$, and denote by $\bar{r}$ the image of an element $r \in R$ under the projection $R \rightarrow R_{q}$.

We clearly have that $J_{q}$ is $\mathrm{GL}_{\alpha^{-}}$and $\mathfrak{g}_{\alpha}$-invariant, so that we can consider the induced actions on $R_{q}=R / J_{q}$. If we let $R_{q}^{t} \subset R_{q}$ denote the subalgebra generated by elements $\operatorname{tr}(\bar{r})$, $r \in R$, we can see that $R_{q}^{t} \subset R_{q}^{\mathrm{GL}_{\alpha}} \cap R_{q}^{\mathfrak{g}_{\alpha}}$. The next result follows either from [20, Proposition 6.8.1] and [6, Theorem 4.5], or from [20, Proposition 7.13.2] and quasi-Hamiltonian reduction [3].

Theorem 5.10 Let $(A,\{\{-,-\}\}, \Phi)$ be a quasi-Hamiltonian algebra over $B$. Then, for any $q \in B^{\times}$, the algebra $R_{q}^{t}$ is a Poisson algebra whose Poisson bracket is induced by the quasi-Poisson bracket on $R$.

Corollary 5.11 Assume that $(A,\{\{-,-\}\}, \Phi)$ is a quasi-Hamiltonian algebra over $B$, and fix $q \in B^{\times}$. If $\mathbb{k}$ is an algebraically closed field of characteristic 0 , then the algebra $R_{q}^{\mathrm{GL}_{\alpha}}=(\mathcal{O}(\operatorname{Rep}(A, \alpha)) /(\mathcal{X}(\Phi-q)))^{\mathrm{GL}_{\alpha}}$ is a Poisson algebra.

Example 5.12 If $\mathbb{k}$ is algebraically closed, the double quasi-Poisson bracket of Van den Bergh given in Theorem 3.3 (with $\gamma_{a}=+1$ for all $a \in \bar{Q}$ ) defines a Poisson structure on multiplicative quiver varieties of Crawley-Boevey and Shaw [7], see [20, Theorem 1.1].

Acknowledgements The author is grateful to O. Chalykh for introducing him to the theory of double brackets, and for valuable comments on an earlier draft of this work which greatly improved the presentation 
of the present paper. The author also thanks A. Alekseev for useful discussions, and the referees for their comments. Part of this work was supported by a University of Leeds 110 Anniversary Research Scholarship.

Open Access This article is licensed under a Creative Commons Attribution 4.0 International License, which permits use, sharing, adaptation, distribution and reproduction in any medium or format, as long as you give appropriate credit to the original author(s) and the source, provide a link to the Creative Commons licence, and indicate if changes were made. The images or other third party material in this article are included in the article's Creative Commons licence, unless indicated otherwise in a credit line to the material. If material is not included in the article's Creative Commons licence and your intended use is not permitted by statutory regulation or exceeds the permitted use, you will need to obtain permission directly from the copyright holder. To view a copy of this licence, visit http://creativecommonshorg/licenses/by/4.0/.

\section{Appendix A: Vanishing of the map $\kappa$}

In this appendix, we prove Lemma 2.20. Note that $\kappa$ is a linear combination of triple brackets, so it is itself a triple bracket. By definition, it is a derivation in its last argument and is cyclically anti-symmetric. Thus, to show that $\kappa$ vanishes, it suffices to show that it is equal to zero when applied to generators of $A^{f}$. Before tackling this task, we use Eq. 1.4 and remark that we can write

$$
\begin{aligned}
& \kappa(-,-,-)=\{-,-,-\}^{f}-\{-,-,-\}-\{-,-,-\}_{f u s} \\
= & \sum_{r \in \mathbb{Z}_{3}} \tau_{(123)}^{r} \circ\left[\left(\{-,-\} \otimes 1_{A}\right) \circ\left(1_{A} \otimes\{-,-\}_{f u s}\right)+\left(\{-,-\}_{f u s} \otimes 1_{A}\right) \circ\left(1_{A} \otimes\{-,-\}\right)\right] \circ \tau_{(123)}^{-r},
\end{aligned}
$$

where $1_{A}$ is the identity map. Therefore, evaluated on some elements $a, b, c \in A^{f}$, we can write

$$
\begin{aligned}
& \kappa(a, b, c)=\underbrace{\left\{\left\{a,\{b, c\}_{f u s}^{\prime}\right\}\right\} \otimes\{b, c\}_{f u s}^{\prime \prime}}_{A}+\underbrace{\left.\left\{a,\{b, c\}^{\prime}\right\}\right\}_{f u s} \otimes\{b, c\}^{\prime \prime}}_{A^{\prime}} \\
& +\underbrace{\tau_{(123)}\left\{\left\{b,\{c, a\}_{f u s}^{\prime}\right\}\right\} \otimes\{c, a\}_{f u s}^{\prime \prime}}_{B}+\underbrace{\tau_{(123)}\left\{b,\{c, a\}^{\prime}\right\}_{f u s} \otimes\{c, a\}^{\prime \prime}}_{B^{\prime}} \\
& +\underbrace{\tau_{(132)}\left\{\left\{c,\{a, b\}_{f u s}^{\prime}\right\}\right\} \otimes\{a, b\}_{f u s}^{\prime \prime}}_{C}+\underbrace{\tau_{(132)}\left\{c c,\{a, b\}^{\prime}\right\}_{f u s} \otimes\{a, b\}^{\prime \prime}}_{C^{\prime}},
\end{aligned}
$$

so that we will write down the terms $A, B, C, A^{\prime}, B^{\prime}, C^{\prime}$ for the different types of generators. Using the cyclicity, we only have twenty cases to check. We will only detail the computations in the first few cases, and we will give the final form of the terms $A, B, C, A^{\prime}, B^{\prime}, C^{\prime}$ in the remaining cases so that the reader can check that they sum up to zero.

Before beginning with the calculations, we remark that identities involving the double bracket $\{-,-\}$ follow from extension from $A$ to $A^{f}$ which respects the derivation property in each variable. That is, given $e_{+}, f_{+} \in\left\{\epsilon, e_{12}\right\}$ and $e_{-}, f_{-} \in\left\{\epsilon, e_{21}\right\}$, we have for any $a=e_{+} \alpha e_{-}, b=f_{+} \beta f_{-}$with $\alpha, \beta \in A$ that

$$
\{a, b\}=f_{+}\{\{\alpha, \beta\}\}^{\prime} e_{-} \otimes e_{+}\left\{\{\alpha, \beta\}^{\prime \prime} f_{-} .\right.
$$

Here, in the left-hand side we have the induced double bracket on $A^{f}$, while the double bracket in the right-hand side is the original one on $A$. Recall that we can choose generators $a, b \in A^{f}$ that admit such a decomposition by Lemma 2.11 . 


\section{A.1 All generators of the same type}

We drop the idempotent $\epsilon$ in our computations since this is the unit in $A^{f}$.

Generators of the second type. Write $a=e_{12} \alpha, b=e_{12} \beta$ and $c=e_{12} \gamma$ for $\alpha, \beta, \gamma \in e_{2} A \epsilon$. Using Eq. 2.15b, then the derivation property for the outer bimodule structure in the second entry of the double bracket on $A^{f}$ together with Eq. A.2, we get that

$$
\begin{aligned}
A(a, b, c) & =-\frac{1}{2}\left\{\left\{e_{12} \alpha, e_{12} \gamma e_{12} \beta\right\} \otimes e_{1}=-\frac{1}{2}\left(e_{12} \gamma\left\{e_{12} \alpha, e_{12} \beta\right\}+\left\{e_{12} \alpha, e_{12} \gamma\right\} e_{12} \beta\right) \otimes e_{1}\right. \\
& \left.=-\frac{1}{2} e_{12}\{\alpha, \gamma\}^{\prime} \otimes e_{12}\{\alpha, \gamma\}^{\prime \prime} e_{12} \beta \otimes e_{1}-\frac{1}{2} e_{12} \gamma e_{12}\{\alpha \alpha, \beta\}^{\prime} \otimes e_{12}\{\alpha, \beta\}\right\}^{\prime \prime} \otimes e_{1} .
\end{aligned}
$$

Similarly we obtain

$$
\begin{aligned}
B(a, b, c) & =-\frac{1}{2} \tau_{(123)}\left(e _ { 1 2 } \left\{\{ \beta , \alpha \} ^ { \prime } \otimes e _ { 1 2 } \left\{\{\beta, \alpha\}^{\prime \prime} e_{12} \gamma \otimes e_{1}+e_{12} \alpha e_{12}\left\{\{\beta, \gamma\}^{\prime} \otimes e_{12}\{\beta, \gamma\}^{\prime \prime} \otimes e_{1}\right)\right.\right.\right. \\
& =-\frac{1}{2} e_{1} \otimes e_{12}\left\{\{\beta, \alpha\}^{\prime} \otimes e_{12}\{\beta, \alpha\}^{\prime \prime} e_{12} \gamma-\frac{1}{2} e_{1} \otimes e_{12} \alpha e_{12}\left\{\{ \beta , \gamma \} ^ { \prime } \otimes e _ { 1 2 } \left\{\{\beta, \gamma\}^{\prime \prime},\right.\right.\right. \\
C(a, b, c) & =-\frac{1}{2} \tau_{(132)}\left(e_{12}\{\gamma, \beta\}^{\prime} \otimes e_{12}\{\gamma \gamma, \beta\}^{\prime \prime} e_{12} \alpha \otimes e_{1}+e_{12} \beta e_{12}\{\gamma, \alpha\}^{\prime} \otimes e_{12}\{\gamma, \alpha\}^{\prime \prime} \otimes e_{1}\right) \\
& =-\frac{1}{2} e_{12}\{\gamma, \beta\}^{\prime \prime} e_{12} \alpha \otimes e_{1} \otimes e_{12}\left\{\{\gamma, \beta\}^{\prime}-\frac{1}{2} e_{12}\{\gamma, \alpha\}^{\prime \prime} \otimes e_{1} \otimes e_{12} \beta e_{12}\{\gamma, \alpha\}^{\prime} .\right.
\end{aligned}
$$

Now, remark that Eq. A.2 gives $\left\{e_{12} \beta, e_{12} \gamma\right\}=e_{12}\{\beta \beta, \gamma\}^{\prime} \otimes e_{12}\{\{\beta, \gamma\}\}^{\prime \prime}$, so that the element in the first copy of $A^{\otimes 2}$ is also a generator of the second type. Using Eq. 2.15b for the expression of $\{-,-\}_{f u s}$, we get

$$
\begin{aligned}
A^{\prime}(a, b, c) & =\left\{\left\{e_{12} \alpha, e_{12}\{\{\beta, \gamma\}\}^{\prime}\right\}\right\}_{f u s} \otimes e_{12}\{\beta, \gamma\}^{\prime \prime} \\
& \left.=\frac{1}{2} e_{1} \otimes e_{12} \alpha e_{12}\{\beta, \gamma\}^{\prime} \otimes e_{12}\{\beta, \gamma\}^{\prime \prime}-\frac{1}{2} e_{12}\{\beta, \gamma\}^{\prime} e_{12} \alpha \otimes e_{1} \otimes e_{12}\{\beta, \gamma\}\right\}^{\prime \prime} .
\end{aligned}
$$

In the same way, we find

$$
\begin{aligned}
B^{\prime}(a, b, c) & =\frac{1}{2} \tau_{(123)}\left(e_{1} \otimes e_{12} \beta e_{12}\{\gamma, \alpha\}^{\prime} \otimes e_{12}\{\gamma, \alpha\}^{\prime \prime}-e_{12}\{\gamma, \alpha\}^{\prime} e_{12} \beta \otimes e_{1} \otimes e_{12}\{\gamma, \alpha\}^{\prime \prime}\right) \\
& =\frac{1}{2} e_{12}\{\gamma, \alpha\}^{\prime \prime} \otimes e_{1} \otimes e_{12} \beta e_{12}\{\gamma, \alpha\}^{\prime}-\frac{1}{2} e_{12}\{\gamma, \alpha\}^{\prime \prime} \otimes e_{12}\{\gamma, \alpha\}^{\prime} e_{12} \beta \otimes e_{1}, \\
C^{\prime}(a, b, c) & =\frac{1}{2} \tau_{(132)}\left(e_{1} \otimes e_{12} \gamma e_{12}\left\{\{\alpha, \beta\}^{\prime} \otimes e_{12}\{\alpha \alpha, \beta\}^{\prime \prime}-e_{12}\{\alpha \alpha, \beta\}\right\}^{\prime} e_{12} \gamma \otimes e_{1} \otimes e_{12}\{\alpha, \beta\}^{\prime \prime}\right) \\
& =\frac{1}{2} e_{12} \gamma e_{12}\{\alpha, \beta\}^{\prime} \otimes e_{12}\{\alpha \alpha, \beta\}^{\prime \prime} \otimes e_{1}-\frac{1}{2} e_{1} \otimes e_{12}\{\alpha \alpha, \beta\}^{\prime \prime} \otimes e_{12}\{\alpha, \beta\}^{\prime} e_{12} \gamma .
\end{aligned}
$$

Summing all terms, we obtain after obvious cancellations

$$
\begin{aligned}
\kappa(a, b, c)= & -\frac{1}{2}\left(e_{12}\{\alpha, \gamma\}^{\prime} \otimes e_{12}\{\alpha, \gamma\}^{\prime \prime} e_{12} \beta \otimes e_{1}+e_{12}\{\gamma, \alpha\}^{\prime \prime} \otimes e_{12}\{\gamma, \alpha\}^{\prime} e_{12} \beta \otimes e_{1}\right) \\
& -\frac{1}{2}\left(e _ { 1 } \otimes e _ { 1 2 } \left\{\{\beta, \alpha\}^{\prime} \otimes e_{12}\left\{\{\beta, \alpha\}^{\prime \prime} e_{12} \gamma+e_{1} \otimes e_{12}\{\alpha \alpha, \beta\}^{\prime \prime} \otimes e_{12}\{\alpha, \beta\}^{\prime} e_{12} \gamma\right)\right.\right. \\
& -\frac{1}{2}\left(e_{12}\{\gamma, \beta\}^{\prime \prime} e_{12} \alpha \otimes e_{1} \otimes e_{12}\{\gamma, \beta\}^{\prime}+e_{12}\left\{\{\beta, \gamma\}^{\prime} e_{12} \alpha \otimes e_{1} \otimes e_{12}\{\beta, \gamma\}^{\prime \prime}\right) .\right.
\end{aligned}
$$

It remains to notice in the last expression that all lines vanish using the cyclic antisymmetry of the double bracket. 
Generators of the third type. Write $a=\alpha e_{21}, b=\beta e_{21}$ and $c=\gamma e_{21}$ for $\alpha, \beta, \gamma \in \epsilon A e_{2}$. From Eqs. 2.16c and A.2 we get that

$$
\begin{aligned}
A(a, b, c) & \left.=\frac{1}{2}\left\{\alpha \alpha e_{21}, \gamma e_{21} \beta e_{21}\right\}\right\} \otimes e_{1} \\
& =\frac{1}{2}\{\alpha, \gamma\}^{\prime} e_{21} \otimes\{\alpha, \gamma\}^{\prime \prime} e_{21} \beta e_{21} \otimes e_{1}+\frac{1}{2} \gamma e_{21}\{\alpha, \beta\}^{\prime} e_{21} \otimes\{\alpha, \beta\}^{\prime \prime} e_{21} \otimes e_{1} .
\end{aligned}
$$

Similarly we obtain

$$
\begin{aligned}
& B(a, b, c)=\frac{1}{2} e_{1} \otimes\{\beta, \alpha\}^{\prime} e_{21} \otimes\{\beta, \alpha\}^{\prime \prime} e_{21} \gamma e_{21}+\frac{1}{2} e_{1} \otimes \alpha e_{21}\left\{\{ \beta , \gamma \} ^ { \prime } e _ { 2 1 } \otimes \left\{\{\beta, \gamma\}^{\prime \prime} e_{21},\right.\right. \\
& C(a, b, c)=\frac{1}{2}\left\{\{\gamma, \beta\}^{\prime \prime} e_{21} \alpha e_{21} \otimes e_{1} \otimes\{\gamma, \beta\}^{\prime} e_{21}+\frac{1}{2}\{\gamma, \alpha\}^{\prime \prime} e_{21} \otimes e_{1} \otimes \beta e_{21}\{\gamma, \alpha\}^{\prime} e_{21} .\right.
\end{aligned}
$$

Noticing from Eq. A.2 that $\{b, c\}^{\prime}=\left\{\{\beta, \gamma\}^{\prime} e_{21}\right.$ is a generator of the third type, we get again from Eq. 2.16c

$$
\begin{aligned}
A^{\prime}(a, b, c) & =\left\{\left\{\alpha e_{21},\{\{\beta, \gamma\}\}^{\prime} e_{21}\right\}\right\}_{f u s} \otimes\{\beta, \gamma\}^{\prime \prime} e_{21} \\
& =\frac{1}{2}\{\{\beta, \gamma\}\}^{\prime} e_{21} \alpha e_{21} \otimes e_{1} \otimes\{\{\beta, \gamma\}\}^{\prime \prime} e_{21}-\frac{1}{2} e_{1} \otimes \alpha e_{21}\{\beta, \gamma\}^{\prime} e_{21} \otimes\{\{\beta, \gamma\}\}^{\prime \prime} e_{21} .
\end{aligned}
$$

Analogously

$$
\begin{aligned}
& B^{\prime}(a, b, c)=\frac{1}{2}\{\gamma, \alpha\}^{\prime \prime} e_{21} \otimes\{\gamma, \alpha\}^{\prime} e_{21} \beta e_{21} \otimes e_{1}-\frac{1}{2}\{\gamma, \alpha\}^{\prime \prime} e_{21} \otimes e_{1} \otimes \beta e_{21}\{\gamma, \alpha\}^{\prime} e_{21}, \\
& C^{\prime}(a, b, c)=\frac{1}{2} e_{1} \otimes\{\alpha, \beta\}^{\prime \prime} e_{21} \otimes\{\alpha \alpha, \beta\}^{\prime} e_{21} \gamma e_{21}-\frac{1}{2} \gamma e_{21}\{\alpha, \beta\}^{\prime} e_{21} \otimes\{\alpha, \beta\}^{\prime \prime} e_{21} \otimes e_{1} .
\end{aligned}
$$

Summing all terms yield

$$
\begin{aligned}
\kappa(a, b, c)= & +\frac{1}{2}\left(\{\alpha, \gamma\}^{\prime} e_{21} \otimes\{\alpha, \gamma\}^{\prime \prime} e_{21} \beta e_{21} \otimes e_{1}+\{\gamma, \alpha\}^{\prime \prime} e_{21} \otimes\{\gamma, \alpha\}^{\prime} e_{21} \beta e_{21} \otimes e_{1}\right) \\
& +\frac{1}{2}\left(e_{1} \otimes\{\beta, \alpha\}^{\prime} e_{21} \otimes\left\{\{\beta, \alpha\}^{\prime \prime} e_{21} \gamma e_{21}+e_{1} \otimes\{\alpha, \beta\}^{\prime \prime} e_{21} \otimes\{\alpha, \beta\}^{\prime} e_{21} \gamma e_{21}\right)\right. \\
& +\frac{1}{2}\left(\{\gamma, \beta\}^{\prime \prime} e_{21} \alpha e_{21} \otimes e_{1} \otimes\{\gamma, \beta\}^{\prime} e_{21}+\left\{\{\beta, \gamma\}^{\prime} e_{21} \alpha e_{21} \otimes e_{1} \otimes\{\beta, \gamma\}^{\prime \prime} e_{21}\right),\right.
\end{aligned}
$$

which is zero using the cyclic antisymmetry.

Generators of the first type. For $a, b, c \in \epsilon A \epsilon$, we have by Eq. A.2 that the double bracket $\{-,-\}$ evaluated on any two of these elements belongs to $(\epsilon A \epsilon)^{\otimes 2}$. At the same time, Eq. 2.14a gives that $\{\epsilon A \epsilon, \epsilon A \epsilon\}_{f u s}=0$. Hence all terms in Eq. A.1 trivially vanish and $\kappa(a, b, c)=0$.

Generators of the fourth type. As in the first type case, we use Eq. A.2 to get that $\left\{e_{12} A e_{21}, e_{12} A e_{21}\right\} \subset\left(e_{12} A e_{21}\right)^{\otimes 2}$ and Eq. $2.17 \mathrm{~d}$ to obtain $\left\{e_{12} A e_{21}, e_{12} A e_{21}\right\}_{f u s}=0$, so that all terms vanish.

\section{A.2 Two generators of the first type}

Let $a, b \in \epsilon A \epsilon$.

With one generator of the second type. Consider $c=e_{12} \gamma$ for some $\gamma \in e_{2} A \epsilon$. Using Eqs. 2.14b and 2.15a,

$$
\begin{aligned}
& A=-\frac{1}{2} e_{1}\{a, b\}^{\prime} \otimes\{a, b\}^{\prime \prime} \otimes e_{12} \gamma, \\
& B=\frac{1}{2} e_{1} a \otimes e_{12}\{b, \gamma\}^{\prime} \otimes\{b, \gamma\}^{\prime \prime}-\frac{1}{2} e_{1} \otimes\{b, a\}^{\prime} \otimes\{b, a\}^{\prime \prime} e_{12} \gamma-\frac{1}{2} e_{1} \otimes a e_{12}\{b, \gamma\}^{\prime} \otimes\{b, \gamma\}^{\prime \prime} .
\end{aligned}
$$


By Eq. 2.14a, $C$ trivially vanishes. It is also the case for $B^{\prime}$ because $\left\{e_{12} \gamma, a\right\}^{\prime} \in \epsilon A \epsilon$. Next we get by Eqs. $2.14 \mathrm{~b}$ and $2.15 \mathrm{a}$ that

$$
\begin{aligned}
& A^{\prime}=\frac{1}{2} e_{1} \otimes a e_{12}\{b, \gamma\}^{\prime} \otimes\{b, \gamma\}^{\prime \prime}-\frac{1}{2} e_{1} a \otimes e_{12}\{b, \gamma\}^{\prime} \otimes\{b, \gamma\}^{\prime \prime}, \\
& C^{\prime}=\frac{1}{2} e_{1}\{a, b\}^{\prime} \otimes\{a, b\}^{\prime \prime} \otimes e_{12} \gamma-\frac{1}{2} e_{1} \otimes\{a, b\}^{\prime \prime} \otimes\{a, b\}^{\prime} e_{12} \gamma,
\end{aligned}
$$

so that all terms cancel out together (after using the cyclic antisymmetry, which we will need in each of the remaining cases).

With one generator of the third type. Consider $c=\gamma e_{21}$ for some $\gamma \in \epsilon A e_{2}$. We get from Eqs. 2.14c and 2.16a that

$$
\begin{aligned}
A= & \frac{1}{2}\{a, \gamma\}^{\prime} \otimes\{a, \gamma\}^{\prime \prime} e_{21} b \otimes e_{1}+\frac{1}{2} \gamma e_{21}\{a, b\}^{\prime} \otimes\{a, b\}^{\prime \prime} \otimes e_{1} \\
& -\frac{1}{2}\{a, \gamma\}^{\prime} \otimes\{a, \gamma\}^{\prime \prime} e_{21} \otimes b e_{1}, \\
B= & \frac{1}{2} \gamma e_{21} \otimes\{b, a\}^{\prime} \otimes\{b, a\}^{\prime \prime} e_{1} .
\end{aligned}
$$

Again using Eq. 2.14a we have $C=0$, and $A^{\prime}=0$ since $\left\{b, \gamma e_{21}\right\}^{\prime} \in \epsilon \in$. Finally, from Eqs. 2.14c and 2.16a we get

$$
\begin{aligned}
& B^{\prime}=\frac{1}{2}\{\gamma, a\}^{\prime \prime} \otimes\{\gamma, a\}^{\prime} e_{21} b \otimes e_{1}-\frac{1}{2}\{\gamma, a\}^{\prime \prime} \otimes\{\gamma, a\}^{\prime} e_{21} \otimes b e_{1}, \\
& C^{\prime}=\frac{1}{2} \gamma e_{21} \otimes\{a, b\}^{\prime \prime} \otimes\{a, b\}^{\prime} e_{1}-\frac{1}{2} \gamma e_{21}\{a, b\}^{\prime} \otimes\{a, b\}^{\prime \prime} \otimes e_{1},
\end{aligned}
$$

and all terms sum up to zero.

With one generator of the fourth type. Consider $c=e_{12} \gamma e_{21}$ for some $\gamma \in e_{2} A e_{2}$. First, using

Eqs. 2.14d and 2.17a we get

$$
\begin{aligned}
A= & \frac{1}{2} e_{12}\{a, \gamma\}^{\prime} \otimes\{a, \gamma\}^{\prime \prime} e_{21} b \otimes e_{1}+\frac{1}{2} e_{12} \gamma e_{21}\{a, b\}^{\prime} \otimes\{a, b\}^{\prime \prime} \otimes e_{1} \\
& -\frac{1}{2} e_{12}\{a, \gamma\}^{\prime} \otimes\{a, \gamma\}^{\prime \prime} e_{21} \otimes b e_{1}-\frac{1}{2} e_{1}\{a, b\}^{\prime} \otimes\{a, b\}^{\prime \prime} \otimes e_{12} \gamma e_{21}, \\
B= & \frac{1}{2} e_{12} \gamma e_{21} \otimes\{b, a\}^{\prime} \otimes\{b, a\}^{\prime \prime} e_{1}+\frac{1}{2} e_{1} a \otimes e_{12}\{b, \gamma\}^{\prime} \otimes\{b, \gamma\}^{\prime \prime} e_{21} \\
& -\frac{1}{2} e_{1} \otimes\{b, a\}^{\prime} \otimes\{b, a\}^{\prime \prime} e_{12} \gamma e_{21}-\frac{1}{2} e_{1} \otimes a e_{12}\{b, \gamma\}^{\prime} \otimes\{b, \gamma\}^{\prime \prime} e_{21} .
\end{aligned}
$$

Again, $C=0$ by Eq. 2.14a. Meanwhile, we find from Eqs. 2.14b, 2.14c and 2.17a

$$
\begin{aligned}
A^{\prime}= & \frac{1}{2} e_{1} \otimes a e_{12}\{b, \gamma\}^{\prime} \otimes\{b, \gamma\}^{\prime \prime} e_{21}-\frac{1}{2} e_{1} a \otimes e_{12}\{b, \gamma\}^{\prime} \otimes\{b, \gamma\}^{\prime \prime} e_{21}, \\
B^{\prime}= & \frac{1}{2} e_{12}\{\gamma, a\}^{\prime \prime} \otimes\{\gamma, a\}^{\prime} e_{21} b \otimes e_{1}-\frac{1}{2} e_{12}\{\gamma, a\}^{\prime \prime} \otimes\{\gamma, a\}^{\prime} e_{21} \otimes b e_{1}, \\
C^{\prime}= & \frac{1}{2} e_{12} \gamma e_{21} \otimes\{a, b\}^{\prime \prime} \otimes\{a, b\}^{\prime} e_{1}+\frac{1}{2} e_{1}\{a, b\}^{\prime} \otimes\{a, b\}^{\prime \prime} \otimes e_{12} \gamma e_{21} \\
& -\frac{1}{2} e_{1} \otimes\{a, b\}^{\prime \prime} \otimes\{a, b\}^{\prime} e_{12} \gamma e_{21}-\frac{1}{2} e_{12} \gamma e_{21}\{a, b\}^{\prime} \otimes\{a, b\}^{\prime \prime} \otimes e_{1} .
\end{aligned}
$$

Summing terms together, we get $\kappa=0$.

\section{A.3 Two generators of the second type}

Let $a=e_{12} \alpha, b=e_{12} \beta$ for $\alpha, \beta \in e_{2} A \epsilon$. We only collect the final form of the terms $A, B, C, A^{\prime}, B^{\prime}, C^{\prime}$ from now on, and the reader can check that they sum up to zero. 
With one generator of the first type. Consider $c \in \epsilon A \epsilon$.

$A=\frac{1}{2} e_{12}\{\alpha \alpha, \beta\}^{\prime} \otimes e_{12}\{\alpha \alpha, \beta\}^{\prime \prime} \otimes e_{1} c-\frac{1}{2}\{\alpha, c\}^{\prime} \otimes e_{12}\{\alpha, c\}^{\prime \prime} e_{12} \beta \otimes e_{1}-\frac{1}{2} c e_{12}\{\alpha, \beta\}^{\prime} \otimes e_{12}\{\alpha \alpha, \beta\}^{\prime \prime} \otimes e_{1}$, $B=-\frac{1}{2} e_{12} \alpha \otimes e_{1}\{\beta, c\}^{\prime} \otimes e_{12}\{\beta, c\}^{\prime \prime}$,

$C=-\frac{1}{2}\{c, \beta\}^{\prime \prime} e_{12} \alpha \otimes e_{1} \otimes e_{12}\{c, \beta\}^{\prime}-\frac{1}{2}\{c, \alpha\}^{\prime \prime} \otimes e_{1} \otimes e_{12} \beta e_{12}\{c, \alpha\}^{\prime}$,

$A^{\prime}=\frac{1}{2} e_{12} \alpha \otimes e_{1}\{\beta, c\}^{\prime} \otimes e_{12}\left\{\{\beta, c\}^{\prime \prime}-\frac{1}{2}\left\{\{\beta, c\}^{\prime} e_{12} \alpha \otimes e_{1} \otimes e_{12}\{\beta \beta, c\}^{\prime \prime}\right.\right.$,

$B^{\prime}=\frac{1}{2}\{c, \alpha\}^{\prime \prime} \otimes e_{1} \otimes e_{12} \beta e_{12}\{c, \alpha\}^{\prime}-\frac{1}{2}\{c, \alpha\}^{\prime \prime} \otimes e_{12}\{c, \alpha\}^{\prime} e_{12} \beta \otimes e_{1}$,

$C^{\prime}=\frac{1}{2} c e_{12}\{\alpha \alpha, \beta\}^{\prime} \otimes e_{12}\{\alpha \alpha, \beta\}^{\prime \prime} \otimes e_{1}-\frac{1}{2} e_{12}\{\alpha, \beta\}^{\prime} \otimes e_{12}\{\alpha \alpha, \beta\}^{\prime \prime} \otimes e_{1} c$.

With one generator of the third type. Consider $c=\gamma e_{21}$ for some $\gamma \in \epsilon e_{2}$.

$$
\begin{aligned}
& A=\frac{1}{2} e_{12}\{\alpha \alpha, \beta\}^{\prime} \otimes e_{12}\{\alpha, \beta\}^{\prime \prime} \otimes e_{1} \gamma e_{21}-\frac{1}{2}\{\alpha, \gamma\}^{\prime} \otimes e_{12}\{\alpha, \gamma\}^{\prime \prime} e_{21} \otimes e_{12} \beta e_{1}, \\
& B=\frac{1}{2} \gamma e_{21} \otimes e_{12}\left\{\{ \beta , \alpha \} ^ { \prime } \otimes e _ { 1 2 } \left\{\{\beta, \alpha\}^{\prime \prime} e_{1}-\frac{1}{2} e_{12} \alpha \otimes e_{1}\{\beta, \gamma\}^{\prime} \otimes e_{12}\left\{\{\beta, \gamma\}^{\prime \prime} e_{21},\right.\right.\right. \\
& C=-\frac{1}{2}\left\{\{\gamma, \beta\}^{\prime \prime} e_{12} \alpha \otimes e_{1} \otimes e_{12}\{\gamma, \beta\}^{\prime} e_{21}-\frac{1}{2}\{\gamma, \alpha\}^{\prime \prime} \otimes e_{1} \otimes e_{12} \beta e_{12}\{\gamma, \alpha\}^{\prime} e_{21},\right. \\
& A^{\prime}=\frac{1}{2} e_{12} \alpha \otimes e_{1}\left\{\{ \beta , \gamma \} ^ { \prime } \otimes e _ { 1 2 } \left\{\{\beta, \gamma\}^{\prime \prime} e_{21}-\frac{1}{2}\left\{\{\beta, \gamma\}^{\prime} e_{12} \alpha \otimes e_{1} \otimes e_{12}\{\beta, \gamma\}^{\prime \prime} e_{21},\right.\right.\right. \\
& B^{\prime}=\frac{1}{2}\{\gamma, \alpha\}^{\prime \prime} \otimes e_{1} \otimes e_{12} \beta e_{12}\{\gamma, \alpha\}^{\prime} e_{21}-\frac{1}{2}\{\gamma, \alpha\}^{\prime \prime} \otimes e_{12}\{\gamma, \alpha\}^{\prime} e_{21} \otimes e_{12} \beta e_{1}, \\
& C^{\prime}=\frac{1}{2} \gamma e_{21} \otimes e_{12}\{\alpha, \beta\}^{\prime \prime} \otimes e_{12}\{\alpha, \beta\}^{\prime} e_{1}-\frac{1}{2} e_{12}\{\alpha, \beta\}^{\prime} \otimes e_{12}\{\alpha, \beta\}^{\prime \prime} \otimes e_{1} \gamma e_{21} .
\end{aligned}
$$

With one generator of the fourth type. Consider $c=e_{12} \gamma e_{21}$ for some $\gamma \in e_{2} \mathrm{Ae}_{2}$.

$A=-\frac{1}{2} e_{12}\{\alpha, \gamma\}^{\prime} \otimes e_{12}\{\alpha, \gamma\}^{\prime \prime} e_{21} \otimes e_{12} \beta e_{1}$,

$B=\frac{1}{2} e_{12} \gamma e_{21} \otimes e_{12}\left\{\{\beta, \alpha\}^{\prime} \otimes e_{12}\{\beta, \alpha\}^{\prime \prime} e_{1}-\frac{1}{2} e_{1} \otimes e_{12}\{\beta, \alpha\}^{\prime} \otimes e_{12}\left\{\{\beta, \alpha\}^{\prime \prime} e_{12} \gamma e_{21}\right.\right.$ $-\frac{1}{2} e_{1} \otimes e_{12} \alpha e_{12}\left\{\{\beta, \gamma\}^{\prime} \otimes e_{12}\left\{\{\beta, \gamma\}^{\prime \prime} e_{21}\right.\right.$,

$C=-\frac{1}{2} e_{12}\{\gamma, \beta\}^{\prime \prime} e_{12} \alpha \otimes e_{1} \otimes e_{12}\{\gamma, \beta\}^{\prime} e_{21}-\frac{1}{2} e_{12}\{\gamma, \alpha\}^{\prime \prime} \otimes e_{1} \otimes e_{12} \beta e_{12}\{\gamma, \alpha\}^{\prime} e_{21}$, $A^{\prime}=\frac{1}{2} e_{1} \otimes e_{12} \alpha e_{12}\left\{\{\beta, \gamma\}^{\prime} \otimes e_{12}\left\{\{\beta, \gamma\}^{\prime \prime} e_{21}-\frac{1}{2} e_{12}\{\beta, \gamma\}^{\prime} e_{12} \alpha \otimes e_{1} \otimes e_{12}\{\beta, \gamma\}^{\prime \prime} e_{21}\right.\right.$, $B^{\prime}=\frac{1}{2} e_{12}\{\gamma, \alpha\}^{\prime \prime} \otimes e_{1} \otimes e_{12} \beta e_{12}\{\gamma, \alpha\}^{\prime} e_{21}-\frac{1}{2} e_{12}\{\gamma, \alpha\}^{\prime \prime} \otimes e_{12}\{\gamma, \alpha\}^{\prime} e_{21} \otimes e_{12} \beta e_{1}$,

$C^{\prime}=\frac{1}{2} e_{12} \gamma e_{21} \otimes e_{12}\{\alpha, \beta\}^{\prime \prime} \otimes e_{12}\{\alpha, \beta\}^{\prime} e_{1}-\frac{1}{2} e_{1} \otimes e_{12}\{\alpha, \beta\}^{\prime \prime} \otimes e_{12}\{\alpha, \beta\}^{\prime} e_{12} \gamma e_{21}$.

\section{A.4 Two generators of the third type}

Let $a=\alpha e_{21}, b=\beta e_{21}$ for $\alpha, \beta \in \epsilon A e_{2}$. 
With one generator of the first type. Consider $c \in \epsilon \in$.

$A=\frac{1}{2}\{\alpha, c\}^{\prime} e_{21} \otimes\{\alpha, c\}^{\prime \prime} e_{1} \otimes \beta e_{21}$,

$B=\frac{1}{2} e_{1} \otimes\left\{\{\beta, \alpha\}^{\prime} e_{21} \otimes\{\beta, \alpha\}^{\prime \prime} e_{21} c+\frac{1}{2} e_{1} \otimes \alpha e_{21}\left\{\{\beta, c\}^{\prime} e_{21} \otimes\{\beta, c\}^{\prime \prime}-\frac{1}{2} c e_{1} \otimes\{\beta, \alpha\}^{\prime} e_{21} \otimes\{\beta, \alpha\}^{\prime \prime} e_{21}\right.\right.$,

$C=\frac{1}{2}\left\{[c, \beta\}^{\prime \prime} e_{21} \alpha e_{21} \otimes e_{1} \otimes\left\{\{c, \beta\}^{\prime}+\frac{1}{2}\{c, \alpha\}^{\prime \prime} e_{21} \otimes e_{1} \otimes \beta e_{21}\{c, \alpha\}^{\prime}\right.\right.$,

$A^{\prime}=\frac{1}{2}\left\{\{\beta, c\}^{\prime} e_{21} \alpha e_{21} \otimes e_{1} \otimes\{\beta, c\}^{\prime \prime}-\frac{1}{2} e_{1} \otimes \alpha e_{21}\left\{\{\beta, c\}^{\prime} e_{21} \otimes\{\beta, c\}^{\prime \prime}\right.\right.$,

$B^{\prime}=\frac{1}{2}\{c, \alpha\}^{\prime \prime} e_{21} \otimes\{c, \alpha\}^{\prime} e_{1} \otimes \beta e_{21}-\frac{1}{2}\{c, \alpha\}^{\prime \prime} e_{21} \otimes e_{1} \otimes \beta e_{21}\{c, \alpha\}^{\prime}$,

$C^{\prime}=\frac{1}{2} e_{1} \otimes\{\alpha, \beta\}^{\prime \prime} e_{21} \otimes\left\{\{\alpha, \beta\}^{\prime} e_{21} c-\frac{1}{2} c e_{1} \otimes\{\alpha \alpha, \beta\}^{\prime \prime} e_{21} \otimes\{\alpha, \beta\}^{\prime} e_{21}\right.$.

With one generator of the second type. Consider $c=e_{12} \gamma$ for some $\gamma \in e_{2} A \epsilon$.

$$
\begin{aligned}
& A=\frac{1}{2} e_{12}\{\alpha, \gamma\}^{\prime} e_{21} \otimes\{\alpha, \gamma\}^{\prime \prime} e_{1} \otimes \beta e_{21}-\frac{1}{2} e_{1}\{\alpha, \beta\}^{\prime} e_{21} \otimes\{\alpha, \beta\}^{\prime \prime} e_{21} \otimes e_{12} \gamma, \\
& B=\frac{1}{2} e_{1} \alpha e_{21} \otimes e_{12}\left\{\{\beta, \gamma\}^{\prime} e_{21} \otimes\{\beta, \gamma\}^{\prime \prime}-\frac{1}{2} e_{12} \gamma e_{1} \otimes\{\beta, \alpha\}^{\prime} e_{21} \otimes\{\beta, \alpha\}^{\prime \prime} e_{21},\right. \\
& C=\frac{1}{2} e_{12}\{\gamma, \beta\}^{\prime \prime} e_{21} \alpha e_{21} \otimes e_{1} \otimes\{\gamma, \beta\}^{\prime}+\frac{1}{2} e_{12}\{\gamma, \alpha\}^{\prime \prime} e_{21} \otimes e_{1} \otimes \beta e_{21}\{\gamma, \alpha\}^{\prime}, \\
& A^{\prime}=\frac{1}{2} e_{12}\{\beta, \gamma\}^{\prime} e_{21} \alpha e_{21} \otimes e_{1} \otimes\{\beta, \gamma\}^{\prime \prime}-\frac{1}{2} e_{1} \alpha e_{21} \otimes e_{12}\left\{\{\beta, \gamma\}^{\prime} e_{21} \otimes\{\beta, \gamma\}^{\prime \prime},\right. \\
& B^{\prime}=\frac{1}{2} e_{12}\{\gamma, \alpha\}^{\prime \prime} e_{21} \otimes\{\gamma, \alpha\}^{\prime} e_{1} \otimes \beta e_{21}-\frac{1}{2} e_{12}\{\gamma, \alpha\}^{\prime \prime} e_{21} \otimes e_{1} \otimes \beta e_{21}\{\gamma, \alpha\}^{\prime}, \\
& C^{\prime}=\frac{1}{2} e_{1}\{\alpha, \beta\}^{\prime} e_{21} \otimes\{\alpha, \beta\}^{\prime \prime} e_{21} \otimes e_{12} \gamma-\frac{1}{2} e_{12} \gamma e_{1} \otimes\{\alpha, \beta\}^{\prime \prime} e_{21} \otimes\{\alpha, \beta\}^{\prime} e_{21} .
\end{aligned}
$$

With one generator of the fourth type. Consider $c=e_{12} \gamma e_{21}$ for some $\gamma \in e_{2} \mathrm{Ae}_{2}$.

$$
\begin{aligned}
A= & \frac{1}{2} e_{12}\{\alpha, \gamma\}^{\prime} e_{21} \otimes\{\alpha, \gamma\}^{\prime \prime} e_{21} \beta e_{21} \otimes e_{1}+\frac{1}{2} e_{12} \gamma e_{21}\{\alpha, \beta\}^{\prime} e_{21} \otimes\{\alpha, \beta\}^{\prime \prime} e_{21} \otimes e_{1} \\
& -\frac{1}{2} e_{1}\{\alpha \alpha, \beta\}^{\prime} e_{21} \otimes\{\alpha, \beta\}^{\prime \prime} e_{21} \otimes e_{12} \gamma e_{21}, \\
B= & \frac{1}{2} e_{1} \alpha e_{21} \otimes e_{12}\left\{\{\beta, \gamma\}^{\prime} e_{21} \otimes\{\beta, \gamma\}^{\prime \prime} e_{21},\right. \\
C= & \frac{1}{2} e_{12}\left\{\{\gamma, \beta\}^{\prime \prime} e_{21} \alpha e_{21} \otimes e_{1} \otimes\{\gamma, \beta\}^{\prime} e_{21}+\frac{1}{2} e_{12}\{\gamma, \alpha\}^{\prime \prime} e_{21} \otimes e_{1} \otimes \beta e_{21}\{\gamma, \alpha\}^{\prime} e_{21},\right. \\
A^{\prime}= & \frac{1}{2} e_{12}\left\{\{\beta, \gamma\}^{\prime} e_{21} \alpha e_{21} \otimes e_{1} \otimes\{\beta, \gamma\}^{\prime \prime} e_{21}-\frac{1}{2} e_{1} \alpha e_{21} \otimes e_{12}\left\{\{\beta, \gamma\}^{\prime} e_{21} \otimes\{\beta, \gamma\}^{\prime \prime} e_{21},\right.\right. \\
B^{\prime}= & \frac{1}{2} e_{12}\{\gamma, \alpha\}^{\prime \prime} e_{21} \otimes\{\gamma, \alpha\}^{\prime} e_{21} \beta e_{21} \otimes e_{1}-\frac{1}{2} e_{12}\{\gamma, \alpha\}^{\prime \prime} e_{21} \otimes e_{1} \otimes \beta e_{21}\{\gamma, \alpha\}^{\prime} e_{21}, \\
C^{\prime}= & \frac{1}{2} e_{1}\left\{\{\alpha, \beta\}^{\prime} e_{21} \otimes\{\alpha \alpha, \beta\}^{\prime \prime} e_{21} \otimes e_{12} \gamma e_{21}-\frac{1}{2} e_{12} \gamma e_{21}\left\{\{\alpha, \beta\}^{\prime} e_{21} \otimes\{\alpha \alpha, \beta\}^{\prime \prime} e_{21} \otimes e_{1} .\right.\right.
\end{aligned}
$$

\section{A.5 Two generators of the fourth type}

Let $a=e_{12} \alpha e_{21}, b=e_{12} \beta e_{21}$ for $\alpha, \beta \in e_{2} A e_{2}$. 
With one generator of the first type. Consider $c \in \epsilon A \epsilon$. We get $C=0$, while

$A=\frac{1}{2}\{\alpha, c\}^{\prime} e_{21} \otimes e_{12}\{\alpha, c\}^{\prime \prime} e_{1} \otimes e_{12} \beta e_{21}+\frac{1}{2} e_{12}\{\alpha, \beta\}^{\prime} e_{21} \otimes e_{12}\{\alpha, \beta\}^{\prime \prime} e_{21} \otimes e_{1} c$ $-\frac{1}{2}\{\alpha, c\}^{\prime} e_{21} \otimes e_{12}\{\alpha, c\}^{\prime \prime} e_{12} \beta e_{21} \otimes e_{1}-\frac{1}{2} c e_{12}\{\alpha \alpha, \beta\}^{\prime} e_{21} \otimes e_{12}\{\alpha \alpha, \beta\}^{\prime \prime} e_{21} \otimes e_{1}$, $B=\frac{1}{2} e_{1} \otimes e_{12}\left\{\{\beta, \alpha\}^{\prime} e_{21} \otimes e_{12}\left\{\{\beta, \alpha\}^{\prime \prime} e_{21} c+\frac{1}{2} e_{1} \otimes e_{12} \alpha e_{21}\left\{\{\beta, c\}^{\prime} e_{21} \otimes e_{12}\left\{\{\beta, c\}^{\prime \prime}\right.\right.\right.\right.$ $-\frac{1}{2} c e_{1} \otimes e_{12}\left\{\{\beta, \alpha\}^{\prime} e_{21} \otimes e_{12}\{\beta, \alpha\}^{\prime \prime} e_{21}-\frac{1}{2} e_{12} \alpha e_{21} \otimes e_{1}\{\beta, c\}^{\prime} e_{21} \otimes e_{12}\{\beta, c\}^{\prime \prime}\right.$,

$\left.\left.A^{\prime}=\frac{1}{2} e_{12} \alpha e_{21} \otimes e_{1}\{\beta, c\}\right\}^{\prime} e_{21} \otimes e_{12}\{\beta, c\}^{\prime \prime}-\frac{1}{2} e_{1} \otimes e_{12} \alpha e_{21}\{\beta, c\}^{\prime} e_{21} \otimes e_{12}\{\beta, c\}\right\}^{\prime \prime}$, $B^{\prime}=\frac{1}{2}\{c, \alpha\}^{\prime \prime} e_{21} \otimes e_{12}\{c, \alpha\}^{\prime} e_{1} \otimes e_{12} \beta e_{21}-\frac{1}{2}\{c, \alpha\}^{\prime \prime} e_{21} \otimes e_{12}\{c, \alpha\}^{\prime} e_{12} \beta e_{21} \otimes e_{1}$, $C^{\prime}=\frac{1}{2} e_{1} \otimes e_{12}\{\alpha, \beta\}^{\prime \prime} e_{21} \otimes e_{12}\left\{\{\alpha, \beta\}^{\prime} e_{21} c+\frac{1}{2} c e_{12}\{\alpha \alpha, \beta\}^{\prime} e_{21} \otimes e_{12}\{\alpha, \beta\}^{\prime \prime} e_{21} \otimes e_{1}\right.$ $-\frac{1}{2} c e_{1} \otimes e_{12}\{\alpha, \beta\}^{\prime \prime} e_{21} \otimes e_{12}\{\alpha, \beta\}^{\prime} e_{21}-\frac{1}{2} e_{12}\{\alpha, \beta\}^{\prime} e_{21} \otimes e_{12}\{\alpha, \beta\}^{\prime \prime} e_{21} \otimes e_{1} c$.

With one generator of the second type. Consider $c=e_{12} \gamma$ for some $\gamma \in e_{2} A \epsilon$. We get $C=0$, $A^{\prime}=0$, while $A=\frac{1}{2} e_{12}\{\alpha, \gamma\}^{\prime} e_{21} \otimes e_{12}\{\alpha, \gamma\}^{\prime \prime} e_{1} \otimes e_{12} \beta e_{21}-\frac{1}{2} e_{12}\{\alpha, \gamma\}^{\prime} e_{21} \otimes e_{12}\{\alpha, \gamma\}^{\prime \prime} e_{12} \beta e_{21} \otimes e_{1}$ $-\frac{1}{2} e_{12} \gamma e_{12}\{\alpha \alpha, \beta\}^{\prime} e_{21} \otimes e_{12}\{\alpha \alpha, \beta\}^{\prime \prime} e_{21} \otimes e_{1}$, $B=-\frac{1}{2} e_{12} \gamma e_{1} \otimes e_{12}\left\{\{\beta, \alpha\}^{\prime} e_{21} \otimes e_{12}\{\beta, \alpha\}^{\prime \prime} e_{21}\right.$, $B^{\prime}=\frac{1}{2} e_{12}\{\gamma, \alpha\}^{\prime \prime} e_{21} \otimes e_{12}\{\gamma, \alpha\}^{\prime} e_{1} \otimes e_{12} \beta e_{21}-\frac{1}{2} e_{12}\{\gamma, \alpha\}^{\prime \prime} e_{21} \otimes e_{12}\{\gamma, \alpha\}^{\prime} e_{12} \beta e_{21} \otimes e_{1}$, $C^{\prime}=\frac{1}{2} e_{12} \gamma e_{12}\{\alpha, \beta\}^{\prime} e_{21} \otimes e_{12}\{\alpha \alpha, \beta\}^{\prime \prime} e_{21} \otimes e_{1}-\frac{1}{2} e_{12} \gamma e_{1} \otimes e_{12}\{\alpha \alpha, \beta\}^{\prime \prime} e_{21} \otimes e_{12}\{\alpha, \beta\}^{\prime} e_{21}$.

With one generator of the third type. Consider $c=\gamma e_{21}$ for some $\gamma \in \epsilon A e_{2}$. We get $C=0$, $B^{\prime}=0$, while $A=\frac{1}{2} e_{12}\{\alpha \alpha, \beta\}^{\prime} e_{21} \otimes e_{12}\{\alpha \alpha, \beta\}^{\prime \prime} e_{21} \otimes e_{1} \gamma e_{21}$, $B=\frac{1}{2} e_{1} \otimes e_{12}\left\{\{\beta, \alpha\}^{\prime} e_{21} \otimes e_{12}\left\{\{\beta, \alpha\}^{\prime \prime} e_{21} \gamma e_{21}+\frac{1}{2} e_{1} \otimes e_{12} \alpha e_{21}\{\beta, \gamma\}^{\prime} e_{21} \otimes e_{12}\{\beta, \gamma\}^{\prime \prime} e_{21}\right.\right.$ $-\frac{1}{2} e_{12} \alpha e_{21} \otimes e_{1}\{\beta, \gamma\}^{\prime} e_{21} \otimes e_{12}\left\{\{\beta, \gamma\}^{\prime \prime} e_{21}\right.$,

$A^{\prime}=\frac{1}{2} e_{12} \alpha e_{21} \otimes e_{1}\{\beta, \gamma\}^{\prime} e_{21} \otimes e_{12}\{\beta, \gamma\}^{\prime \prime} e_{21}-\frac{1}{2} e_{1} \otimes e_{12} \alpha e_{21}\{\beta, \gamma\}^{\prime} e_{21} \otimes e_{12}\{\beta, \gamma\}^{\prime \prime} e_{21}$, $C^{\prime}=\frac{1}{2} e_{1} \otimes e_{12}\left\{\{\alpha, \beta\}^{\prime \prime} e_{21} \otimes e_{12}\{\alpha \alpha, \beta\}^{\prime} e_{21} \gamma e_{21}-\frac{1}{2} e_{12}\{\alpha \alpha, \beta\}^{\prime} e_{21} \otimes e_{12}\{\alpha \alpha, \beta\}^{\prime \prime} e_{21} \otimes e_{1} \gamma e_{21}\right.$.

\section{A.6 Remaining cases}

We now take three different types of generators. 
No generator of the fourth type. Let $a \in \epsilon A \epsilon, b=e_{12} \beta$ for $\beta \in e_{2} A \epsilon$ and $c=\gamma e_{21}$ for $\gamma \in$ $\epsilon A e_{2}$. We have $A^{\prime}=0$, while

$$
\begin{aligned}
A & =\frac{1}{2} e_{12}\left\{\{a, \beta\}^{\prime} \otimes\{a, \beta\}^{\prime \prime} \otimes e_{1} \gamma e_{21}-\frac{1}{2}\{a, \gamma\}^{\prime} \otimes\{a, \gamma\}^{\prime \prime} e_{21} \otimes e_{12} \beta e_{1},\right. \\
B & =\frac{1}{2} \gamma e_{21} \otimes\left\{\{ \beta , a \} ^ { \prime } \otimes e _ { 1 2 } \left\{\{\beta, a\}^{\prime \prime} e_{1},\right.\right. \\
C & =-\frac{1}{2}\{\gamma, a\}^{\prime \prime} \otimes e_{12} \beta \otimes e_{1}\{\gamma, a\}^{\prime} e_{21}, \\
B^{\prime} & =\frac{1}{2}\{\gamma, a\}^{\prime \prime} \otimes e_{12} \beta \otimes e_{1}\{\gamma, a\}^{\prime} e_{21}-\frac{1}{2}\{\gamma, a\}^{\prime \prime} \otimes\{\gamma, a\}^{\prime} e_{21} \otimes e_{12} \beta e_{1}, \\
C^{\prime} & =\frac{1}{2} \gamma e_{21} \otimes\{a, \beta\}^{\prime \prime} \otimes e_{12}\left\{\{a, \beta\}^{\prime} e_{1}-\frac{1}{2} e_{12}\{a, \beta\}^{\prime} \otimes\{a a, \beta\}^{\prime \prime} \otimes e_{1} \gamma e_{21} .\right.
\end{aligned}
$$

No generator of the third type. Let $a \in \epsilon A \epsilon, b=e_{12} \beta$ for $\beta \in e_{2} A \epsilon$ and $c=e_{12} \gamma e_{21}$ for $\gamma \in e_{2} \mathrm{Ae}_{2}$.

$$
\begin{aligned}
A= & -\frac{1}{2} e_{12}\{a, \gamma\}^{\prime} \otimes\{a, \gamma\}^{\prime \prime} e_{21} \otimes e_{12} \beta e_{1}, \\
B= & \frac{1}{2} e_{12} \gamma e_{21} \otimes\{\beta, a\}^{\prime} \otimes e_{12}\left\{\{\beta, a\}^{\prime \prime} e_{1}+\frac{1}{2} e_{1} a \otimes e_{12}\left\{\{ \beta , \gamma \} ^ { \prime } \otimes e _ { 1 2 } \left\{\{\beta, \gamma\}^{\prime \prime} e_{21}\right.\right.\right. \\
& -\frac{1}{2} e_{1} \otimes\{\beta, a\}^{\prime} \otimes e_{12}\left\{\{\beta, a\}^{\prime \prime} e_{12} \gamma e_{21}-\frac{1}{2} e_{1} \otimes a e_{12}\left\{\{\beta, \gamma\}^{\prime} \otimes e_{12}\{\beta, \gamma\}^{\prime \prime} e_{21},\right.\right. \\
C= & -\frac{1}{2} e_{12}\{\gamma, a\}^{\prime \prime} \otimes e_{12} \beta \otimes e_{1}\{\gamma, a\}^{\prime} e_{21},
\end{aligned}
$$

$$
\begin{aligned}
& A^{\prime}=\frac{1}{2} e_{1} \otimes a e_{12}\left\{\{ \beta , \gamma \} ^ { \prime } \otimes e _ { 1 2 } \left\{\{\beta, \gamma\}^{\prime \prime} e_{21}-\frac{1}{2} e_{1} a \otimes e_{12}\left\{\{ \beta , \gamma \} ^ { \prime } \otimes e _ { 1 2 } \left\{\{\beta, \gamma\}^{\prime \prime} e_{21},\right.\right.\right.\right. \\
& B^{\prime}=\frac{1}{2} e_{12}\{\gamma, a\}^{\prime \prime} \otimes e_{12} \beta \otimes e_{1}\{\gamma, a\}^{\prime} e_{21}-\frac{1}{2} e_{12}\{\gamma, a\}^{\prime \prime} \otimes\{\gamma, a\}^{\prime} e_{21} \otimes e_{12} \beta e_{1}, \\
& C^{\prime}=\frac{1}{2} e_{12} \gamma e_{21} \otimes\{a, \beta\}^{\prime \prime} \otimes e_{12}\{a, \beta\}^{\prime} e_{1}-\frac{1}{2} e_{1} \otimes\{a, \beta\}^{\prime \prime} \otimes e_{12}\{a, \beta\}^{\prime} e_{12} \gamma e_{21} .
\end{aligned}
$$

No generator of the second type. This case and the next one are a bit tedious. We set $a \in \epsilon A \epsilon$, $b=\beta e_{21}$ for $\beta \in \epsilon A e_{2}$ and $c=e_{12} \gamma e_{21}$ for $\gamma \in e_{2} A e_{2}$.

$$
\begin{aligned}
A= & \frac{1}{2} e_{12}\{a, \gamma\}^{\prime} \otimes\{a, \gamma\}^{\prime \prime} e_{21} \beta e_{21} \otimes e_{1}+\frac{1}{2} e_{12} \gamma e_{21}\{a, \beta\}^{\prime} \otimes\{a, \beta\}^{\prime \prime} e_{21} \otimes e_{1} \\
& -\frac{1}{2} e_{1}\{a, \beta\}^{\prime} \otimes\{a, \beta\}^{\prime \prime} e_{21} \otimes e_{12} \gamma e_{21}, \\
B= & \frac{1}{2} e_{12} \gamma e_{21} \otimes\{\beta, a\}^{\prime} e_{21} \otimes\{\beta, a\}^{\prime \prime} e_{1}+\frac{1}{2} e_{1} a \otimes e_{12}\left\{\{ \beta , \gamma \} ^ { \prime } e _ { 2 1 } \otimes \left\{\{\beta, \gamma\}^{\prime \prime} e_{21}\right.\right. \\
& -\frac{1}{2} e_{1} \otimes\{\beta, a\}^{\prime} e_{21} \otimes\{\beta, a\}^{\prime \prime} e_{12} \gamma e_{21}-\frac{1}{2} e_{1} \otimes a e_{12}\left\{\{\beta, \gamma\}^{\prime} e_{21} \otimes\{\beta, \gamma\}^{\prime \prime} e_{21},\right. \\
C= & \frac{1}{2} e_{12}\{\gamma, \beta\}^{\prime \prime} e_{21} a \otimes e_{1} \otimes\{\gamma, \beta\}^{\prime} e_{21}+\frac{1}{2} e_{12}\{\gamma, a\}^{\prime \prime} \otimes e_{1} \otimes \beta e_{21}\{\gamma, a\}^{\prime} e_{21} \\
& -\frac{1}{2} e_{12}\{\gamma, \beta\}^{\prime \prime} e_{21} \otimes a e_{1} \otimes\{\gamma, \beta\}^{\prime} e_{21},
\end{aligned}
$$




$$
\begin{aligned}
A^{\prime}= & \frac{1}{2} e_{12}\left\{\{\beta, \gamma\}^{\prime} e_{21} a \otimes e_{1} \otimes\{\beta, \gamma\}^{\prime \prime} e_{21}+\frac{1}{2} e_{1} \otimes a e_{12}\{\{\beta, \gamma\}\}^{\prime} e_{21} \otimes\left\{\{\beta, \gamma\}^{\prime \prime} e_{21}\right.\right. \\
& -\frac{1}{2} e_{12}\left\{\{\beta, \gamma\}^{\prime} e_{21} \otimes a e_{1} \otimes\{\beta, \gamma\}^{\prime \prime} e_{21}-\frac{1}{2} e_{1} a \otimes e_{12}\left\{\{\beta, \gamma\}^{\prime} e_{21} \otimes\{\beta, \gamma\}^{\prime \prime} e_{21},\right.\right. \\
B^{\prime}= & \frac{1}{2} e_{12}\{\gamma, a\}^{\prime \prime} \otimes\{\gamma, a\}^{\prime} e_{21} \beta e_{21} \otimes e_{1}-\frac{1}{2} e_{12}\{\gamma, a\}^{\prime \prime} \otimes e_{1} \otimes \beta e_{21}\{\gamma, a\}^{\prime} e_{21}, \\
C^{\prime}= & \frac{1}{2} e_{12} \gamma e_{21} \otimes\{a, \beta\}^{\prime \prime} e_{21} \otimes\{a, \beta\}^{\prime} e_{1}+\frac{1}{2} e_{1}\{a, \beta\}^{\prime} \otimes\{a, \beta\}^{\prime \prime} e_{21} \otimes e_{12} \gamma e_{21} \\
& \left.-\frac{1}{2} e_{1} \otimes\{a, \beta\}^{\prime \prime} e_{21} \otimes\{a, \beta\}\right\}^{\prime} e_{12} \gamma e_{21}-\frac{1}{2} e_{12} \gamma e_{21}\{a, \beta\}^{\prime} \otimes\{a, \beta\}^{\prime \prime} e_{21} \otimes e_{1} .
\end{aligned}
$$

No generator of the first type. Let $a=e_{12} \alpha$ for $\alpha \in e_{2} A \epsilon, b=\beta e_{21}$ for $\beta \in \epsilon A e_{2}$ and $c=$ $e_{12} \gamma e_{21}$ for $\gamma \in e_{2} A e_{2}$.

$A=\frac{1}{2} e_{12}\{\alpha, \gamma\}^{\prime} \otimes e_{12}\{\alpha, \gamma\}^{\prime \prime} e_{21} \beta e_{21} \otimes e_{1}+\frac{1}{2} e_{12} \gamma e_{21}\{\alpha, \beta\}^{\prime} \otimes e_{12}\{\alpha, \beta\}^{\prime \prime} e_{21} \otimes e_{1}$ $\left.-\frac{1}{2} e_{1}\{\alpha, \beta\}^{\prime} \otimes e_{12}\{\alpha, \beta\}\right\}^{\prime \prime} e_{21} \otimes e_{12} \gamma e_{21}$,

$B=\frac{1}{2} e_{12} \gamma e_{21} \otimes e_{12}\left\{\{\beta, \alpha\}^{\prime} e_{21} \otimes\left\{\{\beta, \alpha\}^{\prime \prime} e_{1}-\frac{1}{2} e_{1} \otimes e_{12}\left\{\{\beta, \alpha\}^{\prime} e_{21} \otimes\{\beta, \alpha\}^{\prime \prime} e_{12} \gamma e_{21}\right.\right.\right.$ $-\frac{1}{2} e_{1} \otimes e_{12} \alpha e_{12}\{\beta, \gamma\}^{\prime} e_{21} \otimes\{\beta, \gamma\}^{\prime \prime} e_{21}$,

$C=\frac{1}{2} e_{12}\{\gamma, \alpha\}^{\prime \prime} \otimes e_{1} \beta e_{21} \otimes e_{12}\{\gamma, \alpha\}^{\prime} e_{21}-\frac{1}{2} e_{12}\{\gamma, \beta\}^{\prime \prime} e_{21} \otimes e_{12} \alpha e_{1} \otimes\{\gamma, \beta\}^{\prime} e_{21}$, $A^{\prime}=\frac{1}{2} e_{1} \otimes e_{12} \alpha e_{12}\{\{\beta, \gamma\}\}^{\prime} e_{21} \otimes\left\{\{\beta, \gamma\}^{\prime \prime} e_{21}-\frac{1}{2} e_{12}\{\beta, \gamma\}^{\prime} e_{21} \otimes e_{12} \alpha e_{1} \otimes\{\beta, \gamma\}^{\prime \prime} e_{21}\right.$, $B^{\prime}=\frac{1}{2} e_{12}\{\gamma, \alpha\}^{\prime \prime} \otimes e_{12}\{\gamma, \alpha\}^{\prime} e_{21} \beta e_{21} \otimes e_{1}-\frac{1}{2} e_{12}\{\gamma, \alpha\}^{\prime \prime} \otimes e_{1} \beta e_{21} \otimes e_{12}\{\gamma, \alpha\}^{\prime} e_{21}$, $C^{\prime}=\frac{1}{2} e_{12} \gamma e_{21} \otimes e_{12}\{\alpha, \beta\}^{\prime \prime} e_{21} \otimes\{\alpha \alpha, \beta\}^{\prime} e_{1}+\frac{1}{2} e_{1}\{\alpha, \beta\}^{\prime} \otimes e_{12}\{\alpha \alpha, \beta\}^{\prime \prime} e_{21} \otimes e_{12} \gamma e_{21}$ $-\frac{1}{2} e_{1} \otimes e_{12}\{\alpha, \beta\}^{\prime \prime} e_{21} \otimes\left\{\{\alpha, \beta\}^{\prime} e_{12} \gamma e_{21}-\frac{1}{2} e_{12} \gamma e_{21}\{\alpha, \beta\}^{\prime} \otimes e_{12}\{\alpha, \beta\}^{\prime \prime} e_{21} \otimes e_{1}\right.$.

\section{Appendix B: Proof of Lemma 2.21}

Note that $\operatorname{Tr}\left(\Phi_{s}\right)=\epsilon \Phi_{s} \epsilon$ for $s \neq 2$, while $\operatorname{Tr}\left(\Phi_{2}\right)=e_{12} \Phi_{2} e_{21}$. In particular, using that for $s \neq 2$ we have $\Phi_{s}=e_{s} \Phi_{s} e_{s}$, we get $\operatorname{Tr}\left(\Phi_{s}\right)=\Phi_{s}$ by understanding that equality in $A^{f}$.

\section{B.1 Moment map condition for the non-fused idempotents}

First, assume that $s \neq 1,2$. Then, using Lemma 2.18, we get

$$
\operatorname{Tr}\left(E_{1}\right)\left(\operatorname{Tr}\left(\Phi_{s}\right)\right)=\Phi_{s} e_{1} \otimes e_{1}-e_{1} \otimes e_{1} \Phi_{s}=0, \quad \operatorname{Tr}\left(E_{2}\right)\left(\operatorname{Tr}\left(\Phi_{s}\right)\right)=0,
$$

which gives $\left\{\operatorname{Tr}\left(\Phi_{s}\right),-\right\}_{f u s}=0$. Therefore, if $a=e_{+} \alpha e_{-}$is a generator of $A^{f}$,

$$
\left\{\operatorname{Tr}\left(\Phi_{s}\right), a\right\}^{f}=\left\{\operatorname{Tr}\left(\Phi_{s}\right), a\right\}=e_{+}\left\{\Phi_{s}, \alpha\right\}^{\prime} \epsilon \otimes \epsilon\left\{\Phi_{s}, \alpha\right\}^{\prime \prime} e_{-},
$$

where the double bracket in the last equality is taken in $A$. By assumption $\Phi_{s}$ satisfies (1.7) for $\{-,-\}$ on $A$ so that

$\left\{\operatorname{Tr}\left(\Phi_{s}\right), a\right\}^{f}=\frac{1}{2}\left(e_{+} \alpha e_{s} \otimes \Phi_{s} e_{-}-e_{+} e_{s} \otimes \Phi_{s} \alpha e_{-}+e_{+} \alpha \Phi_{s} \otimes e_{s} e_{-}-e_{+} \Phi_{s} \otimes e_{s} \alpha e_{-}\right)$, 
where we omitted to write the idempotents $\epsilon$, because with $s \neq 1,2$ we get $e_{s} \epsilon=e_{s}=\epsilon e_{s}$. It remains to see that it coincides with Eq. 2.21 in all four cases of generators. For example, if $a=e_{12} \alpha \epsilon$ with $\alpha \in e_{2} A \epsilon$, we obtain for $e_{+}=e_{12}, e_{-}=\epsilon$

$$
\left\{\operatorname{Tr}\left(\Phi_{s}\right), e_{12} \alpha \epsilon\right\}^{f}=\frac{1}{2}\left(a e_{s} \otimes \Phi_{s}+a \Phi_{s} \otimes e_{s}\right),
$$

because the second and last terms in Eq. B.1 disappear since $e_{12} e_{s}=0=e_{12} \Phi_{s}$. Meanwhile, the right-hand side of Eq. 2.21 reads in that case

$$
\frac{1}{2}\left(a e_{s} \otimes \operatorname{Tr}\left(\Phi_{s}\right)+a \operatorname{Tr}\left(\Phi_{s}\right) \otimes e_{s}-e_{s} \otimes \operatorname{Tr}\left(\Phi_{s}\right) a-\operatorname{Tr}\left(\Phi_{s}\right) \otimes e_{s} a\right),
$$

and the last two terms disappear as $s \neq 1,2$. Indeed $e_{s} a=e_{s} e_{12} \alpha=0$ and $\operatorname{Tr}\left(\Phi_{s}\right) a=$ $\epsilon\left(e_{s} \Phi_{s} e_{s}\right) \epsilon\left(e_{12} \alpha\right)=e_{s} \Phi_{s} e_{s} e_{12} \alpha=0$. The two expressions coincide, and the result is similar with the other types of generators.

\section{B.2 Moment map condition at the fused idempotent}

Using the derivation properties and decomposing the double bracket $\{-,-\}^{f}$ as $\{-,-\}+$ $\{-,-\}_{f u s}$, we obtain for $a=e_{+} \alpha e_{-} \in A^{f}, \alpha \in A$, that

$$
\begin{aligned}
\left\{\left\{\Phi_{1}^{f}, a\right\}\right\}^{f}= & \operatorname{Tr}\left(\Phi_{1}\right) e_{12} * e_{+}\left\{\Phi_{2}, \alpha\right\} e_{-} * e_{21}+\epsilon * e_{+}\left\{\Phi_{1}, \alpha\right\} e_{-} * \epsilon \operatorname{Tr}\left(\Phi_{2}\right) \\
& +\operatorname{Tr}\left(\Phi_{1}\right) *\left\{\operatorname{Tr}\left(\Phi_{2}\right), e_{+} \alpha e_{-}\right\}_{f u s}+\left\{\operatorname{Tr}\left(\Phi_{1}\right), e_{+} \alpha e_{-}\right\}_{f u s} * \operatorname{Tr}\left(\Phi_{2}\right) .
\end{aligned}
$$

The first two terms can easily be obtained from Eq. 1.7. Since $\operatorname{Tr}\left(\Phi_{2}\right)$ is a generator of fourth type (2.6d), we need (2.17a)-(2.17d) to evaluate the third term. In the exact same way, as $\operatorname{Tr}\left(\Phi_{1}\right)$ is a generator of first type (2.6a), we need (2.14a)-(2.14d) to evaluate the last term. Thus, we check separately the four types of generators.

On a generator of the first type. We let $a \in \epsilon A \epsilon$, hence $e_{+}=e_{-}=\epsilon$ and $a=\alpha$. We directly get by Eq. 1.7 that $\left\{\Phi_{2}, a\right\}=0$ since $e_{2} a=0=a e_{2}$, while $\left\{\operatorname{Tr}\left(\Phi_{1}\right), a\right\}_{f u s}=0$ by Eq. 2.14a. For the remaining two terms, we have on one hand by Eq. 1.7

$$
\left\{\Phi_{1}, a\right\}=\frac{1}{2}\left(a e_{1} \otimes \operatorname{Tr}\left(\Phi_{1}\right)-e_{1} \otimes \operatorname{Tr}\left(\Phi_{1}\right) a+a \operatorname{Tr}\left(\Phi_{1}\right) \otimes e_{1}-\operatorname{Tr}\left(\Phi_{1}\right) \otimes e_{1} a\right),
$$

after projecting the equality in $A^{f}$ where $\operatorname{Tr}\left(\Phi_{1}\right)=\Phi_{1}$. On the other hand by Eq. 2.17a $\left\{\operatorname{Tr}\left(\Phi_{2}\right), a\right\}_{f u s}=\frac{1}{2}\left(a e_{1} \otimes \operatorname{Tr}\left(\Phi_{2}\right)+\operatorname{Tr}\left(\Phi_{2}\right) \otimes e_{1} a-a \operatorname{Tr}\left(\Phi_{2}\right) \otimes e_{1}-e_{1} \otimes \operatorname{Tr}\left(\Phi_{2}\right) a\right)$.

Putting this back in Eq. B.2 yields

$$
\begin{aligned}
\left\{\left\{\Phi_{1}^{f}, a\right\}\right\}^{f}= & \frac{1}{2}\left(a e_{1} \operatorname{Tr}\left(\Phi_{2}\right) \otimes \operatorname{Tr}\left(\Phi_{1}\right)-e_{1} \operatorname{Tr}\left(\Phi_{2}\right) \otimes \operatorname{Tr}\left(\Phi_{1}\right) a+a \operatorname{Tr}\left(\Phi_{1}\right) \operatorname{Tr}\left(\Phi_{2}\right) \otimes e_{1}-\operatorname{Tr}\left(\Phi_{1}\right) \operatorname{Tr}\left(\Phi_{2}\right) \otimes e_{1} a\right) \\
& +\frac{1}{2}\left(a e_{1} \otimes \operatorname{Tr}\left(\Phi_{1}\right) \operatorname{Tr}\left(\Phi_{2}\right)+\operatorname{Tr}\left(\Phi_{2}\right) \otimes \operatorname{Tr}\left(\Phi_{1}\right) e_{1} a-a \operatorname{Tr}\left(\Phi_{2}\right) \otimes \operatorname{Tr}\left(\Phi_{1}\right) e_{1}-e_{1} \otimes \operatorname{Tr}\left(\Phi_{1}\right) \operatorname{Tr}\left(\Phi_{2}\right) a\right) .
\end{aligned}
$$

Using that $\operatorname{Tr}\left(\Phi_{1}\right)=e_{1} \operatorname{Tr}\left(\Phi_{1}\right) e_{1}$ and $\operatorname{Tr}\left(\Phi_{2}\right)=e_{1} \operatorname{Tr}\left(\Phi_{2}\right) e_{1}$ allows us to conclude after cancellation of the first and seventh terms, and the second and sixth terms.

On a generator of the second type. Let $a=e_{12} \alpha \epsilon$ with $e_{+}=e_{12}, e_{-}=\epsilon, \alpha \in e_{2} A \epsilon$. We get from Eq. 1.7

$$
\left\{\Phi_{1}, \alpha\right\}=\frac{1}{2}\left(\alpha e_{1} \otimes \Phi_{1}+\alpha \Phi_{1} \otimes e_{1}\right), \quad\left\{\Phi_{2}, \alpha\right\}=-\frac{1}{2}\left(e_{2} \otimes \Phi_{2} \alpha+\Phi_{2} \otimes e_{2} \alpha\right),
$$


because $e_{1} \alpha=0$ and $\alpha e_{2}=0$. Meanwhile, Eqs. 2.14b and 2.17b give

$$
\begin{aligned}
& \left\{\operatorname{Tr}\left(\Phi_{1}\right), a\right\}_{f u s}=\frac{1}{2}\left(e_{1} \otimes \operatorname{Tr}\left(\Phi_{1}\right) a-e_{1} \operatorname{Tr}\left(\Phi_{1}\right) \otimes a\right), \\
& \left\{\operatorname{Tr}\left(\Phi_{2}\right), a\right\}_{f u s}=\frac{1}{2}\left(a e_{1} \otimes \operatorname{Tr}\left(\Phi_{2}\right)-a \operatorname{Tr}\left(\Phi_{2}\right) \otimes e_{1}\right) .
\end{aligned}
$$

Hence, Eq. B.2 gives

$$
\begin{aligned}
\left.\left\{\Phi_{1}^{f}, a\right\}\right\}^{f}= & -\frac{1}{2}\left(e_{12} e_{21} \otimes \operatorname{Tr}\left(\Phi_{1}\right) e_{12} \Phi_{2} \alpha+e_{12} \Phi_{2} e_{21} \otimes \operatorname{Tr}\left(\Phi_{1}\right) e_{12} e_{2} \alpha\right) \\
& +\frac{1}{2}\left(e_{12} \alpha e_{1} \operatorname{Tr}\left(\Phi_{2}\right) \otimes \Phi_{1}+e_{12} \alpha \Phi_{1} \operatorname{Tr}\left(\Phi_{2}\right) \otimes e_{1}\right) \\
& +\frac{1}{2}\left(a e_{1} \otimes \operatorname{Tr}\left(\Phi_{1}\right) \operatorname{Tr}\left(\Phi_{2}\right)-a \operatorname{Tr}\left(\Phi_{2}\right) \otimes \operatorname{Tr}\left(\Phi_{1}\right) e_{1}\right) \\
& +\frac{1}{2}\left(e_{1} \operatorname{Tr}\left(\Phi_{2}\right) \otimes \operatorname{Tr}\left(\Phi_{1}\right) a-e_{1} \operatorname{Tr}\left(\Phi_{1}\right) \operatorname{Tr}\left(\Phi_{2}\right) \otimes a\right)
\end{aligned}
$$

This equality holds in $A^{f}$ where $\operatorname{Tr}\left(\Phi_{1}\right)=\Phi_{1}, \operatorname{Tr}\left(\Phi_{2}\right)=e_{12} \Phi_{2} e_{21}$ and $a=e_{12} \alpha$. Thus it is not hard to rewrite all factors in the four first terms as $\operatorname{Tr}\left(\Phi_{s}\right), a$ or the idempotents (we have to note for the first term that $\left.e_{12} \Phi_{2} \alpha=e_{12} \Phi_{2} e_{2} \alpha=e_{12} \Phi_{2} e_{21} e_{12} \alpha=\operatorname{Tr}\left(\Phi_{2}\right) a\right)$. After cancelling out the second (resp. third) with the seventh (resp. sixth) term, we get the desired result. On a generator of the third type. Let $a=\epsilon \alpha e_{21}$ with $e_{+}=\epsilon, e_{-}=e_{21}, \alpha \in \epsilon A e_{2}$. Using Eq. 1.7 yields

$$
\left\{\Phi_{1}, \alpha\right\}=-\frac{1}{2}\left(e_{1} \otimes \Phi_{1} \alpha+\Phi_{1} \otimes e_{1} \alpha\right), \quad\left\{\Phi_{2}, \alpha\right\}=\frac{1}{2}\left(\alpha e_{2} \otimes \Phi_{2}+\alpha \Phi_{2} \otimes e_{2}\right),
$$

because $\alpha e_{1}=0$ and $e_{2} \alpha=0$. From Eqs. 2.14c and 2.17c we obtain

$$
\begin{aligned}
\left\{\operatorname{Tr}\left(\Phi_{1}\right), a\right\}_{f u s} & =\frac{1}{2}\left(a \operatorname{Tr}\left(\Phi_{1}\right) \otimes e_{1}-a \otimes \operatorname{Tr}\left(\Phi_{1}\right) e_{1}\right), \\
\left\{\operatorname{Tr}\left(\Phi_{2}\right), a\right\}_{f u s} & =\frac{1}{2}\left(\operatorname{Tr}\left(\Phi_{2}\right) \otimes e_{1} a-e_{1} \otimes \operatorname{Tr}\left(\Phi_{2}\right) a\right) .
\end{aligned}
$$

Summing everything inside Eq. B.2, we get

$$
\begin{aligned}
\left\{\left\{\Phi_{1}^{f}, a\right\}\right\}^{f}= & \frac{1}{2}\left(\alpha e_{21} \otimes \operatorname{Tr}\left(\Phi_{1}\right) e_{12} \Phi_{2} e_{21}+\alpha \Phi_{2} e_{21} \otimes \operatorname{Tr}\left(\Phi_{1}\right) e_{12} e_{2} e_{21}\right) \\
& -\frac{1}{2}\left(e_{1} \operatorname{Tr}\left(\Phi_{2}\right) \otimes \Phi_{1} \alpha e_{21}+\Phi_{1} \operatorname{Tr}\left(\Phi_{2}\right) \otimes e_{1} \alpha e_{21}\right) \\
& +\frac{1}{2}\left(\operatorname{Tr}\left(\Phi_{2}\right) \otimes \operatorname{Tr}\left(\Phi_{1}\right) e_{1} a-e_{1} \otimes \operatorname{Tr}\left(\Phi_{1}\right) \operatorname{Tr}\left(\Phi_{2}\right) a\right) \\
& +\frac{1}{2}\left(a \operatorname{Tr}\left(\Phi_{1}\right) \operatorname{Tr}\left(\Phi_{2}\right) \otimes e_{1}-a \operatorname{Tr}\left(\Phi_{2}\right) \otimes \operatorname{Tr}\left(\Phi_{1}\right) e_{1}\right)
\end{aligned}
$$

By arguments similar to the previous case, we can rewrite the four first terms using $a, \operatorname{Tr}\left(\Phi_{1}\right), \operatorname{Tr}\left(\Phi_{2}\right)$ and the idempotents $e_{1}, e_{2}$ so that the second and eighth terms cancel out, while the third and fifth terms cancel out. The remaining terms give the desired result.

On a generator of the fourth type. We let $a=e_{12} \alpha e_{21}$ with $e_{+}=e_{12}, e_{-}=e_{21}, \alpha \in e_{2} A e_{2}$. We directly get by Eq. 1.7 that $\left\{\Phi_{1}, a\right\}=0$, and by Eq. 2.17 d that $\left\{\operatorname{Tr}\left(\Phi_{2}\right), a\right\}_{f u s}=0$. For the remaining two terms, we have by Eqs. 1.7 and 2.14d

$$
\begin{aligned}
\left\{\Phi_{2}, \alpha\right\} & =\frac{1}{2}\left(\alpha e_{2} \otimes \Phi_{2}-e_{2} \otimes \Phi_{2} \alpha+\alpha \Phi_{2} \otimes e_{2}-\Phi_{2} \otimes e_{2} \alpha\right), \\
\left\{\operatorname{Tr}\left(\Phi_{1}\right), a\right\}_{f u s} & =\frac{1}{2}\left(a \operatorname{Tr}\left(\Phi_{1}\right) \otimes e_{1}+e_{1} \otimes \operatorname{Tr}\left(\Phi_{1}\right) a-a \otimes \operatorname{Tr}\left(\Phi_{1}\right) e_{1}-e_{1} \operatorname{Tr}\left(\Phi_{1}\right) \otimes a\right) .
\end{aligned}
$$


Thus, we get after some easy manipulations

$$
\begin{aligned}
\left\{\left\{\Phi_{1}^{f}, a\right\}\right\}^{f}= & \frac{1}{2}\left(a \otimes \operatorname{Tr}\left(\Phi_{1}\right) \operatorname{Tr}\left(\Phi_{2}\right)-e_{1} \otimes \operatorname{Tr}\left(\Phi_{1}\right) \operatorname{Tr}\left(\Phi_{2}\right) a+a \operatorname{Tr}\left(\Phi_{2}\right) \otimes \operatorname{Tr}\left(\Phi_{1}\right)-\operatorname{Tr}\left(\Phi_{2}\right) \otimes \operatorname{Tr}\left(\Phi_{1}\right) a\right) \\
& +\frac{1}{2}\left(a \operatorname{Tr}\left(\Phi_{1}\right) \operatorname{Tr}\left(\Phi_{2}\right) \otimes e_{1}+e_{1} \operatorname{Tr}\left(\Phi_{2}\right) \otimes \operatorname{Tr}\left(\Phi_{1}\right) a-a \operatorname{Tr}\left(\Phi_{2}\right) \otimes \operatorname{Tr}\left(\Phi_{1}\right) e_{1}-e_{1} \operatorname{Tr}\left(\Phi_{2}\right) \operatorname{Tr}\left(\Phi_{1}\right) \otimes a\right),
\end{aligned}
$$

from which we can conclude.

\section{Appendix C: Proof of Proposition 4.4}

Note that any $B$-linear double bracket on $A$ of degree at most +4 on generators needs to satisfy

$$
\begin{aligned}
& \{t, t\}=\lambda(t s t \otimes t-t \otimes t s t), \quad\{s, s\}=l(s t s \otimes s-s \otimes s t s), \\
& \{t, s\}=\gamma e_{2} \otimes e_{1}+\alpha_{1}^{\prime} s t \otimes e_{1}+\alpha_{3} e_{2} \otimes t s+\phi_{0} s t s t \otimes e_{1}+\phi_{1} s t \otimes t s+\phi_{2} e_{2} \otimes t s t s,
\end{aligned}
$$

after using that $t=e_{1} t e_{2}, s=e_{2} s e_{1}$ with the cyclic antisymmetry and the derivation rules. Moreover, if $\{-,-\}$ is a double quasi-Poisson bracket it must satisfy (1.6) on generators, and this is easily seen to be equivalent to require that

$$
\begin{aligned}
& \{t, t, t\}=0, \quad\{s, s, s\}=0, \\
& \{t, t, s\}=\frac{1}{4}\left(s t \otimes t \otimes e_{1}-e_{2} \otimes t \otimes t s\right), \\
& \{s, s, t\}=\frac{1}{4}\left(t s \otimes s \otimes e_{2}-e_{1} \otimes s \otimes s t\right) .
\end{aligned}
$$

Lemma C.1 If Eq. C.2a holds, then either $\lambda=l=0$ or

$$
\gamma=0, \quad \phi_{1}=0, \quad \alpha_{1}^{\prime}=-\alpha_{3}, \quad \phi_{0}=-\phi_{2} .
$$

Proof By Eq. 1.4, we have that for any $a \in A$,

$$
\{a, a, a\}=\left(1+\tau_{(123)}+\tau_{(132)}\right)\left\{\left\{a,\{a, a\}^{\prime}\right\} \otimes\{a, a\}^{\prime \prime} .\right.
$$

We first look at the case $a=t$. Using Eq. C.1a, we can find that

$\left\{t,\{t, t\}^{\prime}\right\} \otimes\{t, t\}^{\prime \prime}=\lambda^{2} t s t s t \otimes t \otimes t-\lambda^{2} t \otimes t s t s t \otimes t-\lambda^{2} t s t \otimes t \otimes t s t+\lambda^{2} t \otimes t s t \otimes t s t+t\{t, s\} t \otimes t$.

The first four terms cancel if we take their sum under cyclic permutations, so that we can write

$$
\begin{aligned}
\{t, t, t\}= & \lambda\left(1+\tau_{(123)}+\tau_{(132)}\right) t\{t, s\} t \otimes t \\
= & \lambda\left(1+\tau_{(123)}+\tau_{(132)}\right)\left[\gamma t \otimes t \otimes t+\left(\alpha_{1}^{\prime}+\alpha_{3}\right) t \otimes t \otimes t s t\right. \\
& \left.+\left(\phi_{0}+\phi_{2}\right) t \otimes t \otimes t s t s t+\phi_{1} t \otimes t s t \otimes t s t\right] .
\end{aligned}
$$

Therefore either $\lambda=0$, or the different coefficients vanish i.e. $\gamma=0, \phi_{1}=0$ while $\alpha_{1}^{\prime}=-\alpha_{3}$ and $\phi_{0}=-\phi_{2}$. Doing the computation with $s$ instead of $t$, we need either $l=0$ or the same four conditions.

Lemma C.2 If $\lambda=0$ and Eq. C.2b holds, then

$$
\begin{gathered}
\phi_{0}=0, \quad \phi_{2}=0, \\
\left(\alpha_{1}^{\prime}\right)^{2}=\frac{1}{4}+\phi_{1} \gamma, \quad \alpha_{3}^{2}=\frac{1}{4}+\phi_{1} \gamma . \quad\left(\alpha_{1}^{\prime}-\alpha_{3}\right) \gamma=0, \quad\left(\alpha_{1}^{\prime}-\alpha_{3}\right) \phi_{1}=0 .
\end{gathered}
$$


The same identities are satisfied if $l=0$ and Eq. C.2c holds.

Proof When we compute $\{t, t, s\}$ using Eq. 1.4, we get that the term $(s t)^{3} \otimes t \otimes e_{1}$ only appears with a factor $\phi_{0}^{2}$, and $e_{2} \otimes t \otimes(t s)^{3}$ only appears with a factor $-\phi_{2}^{2}$. Therefore, if Eq. C.2b is satisfied we need $\phi_{0}=\phi_{2}=0$ which gives Eq. C.5a.

Under the conditions from Eq. C.5a, the only terms remaining in $\{t, t, s\}$ are given by $s t \otimes t \otimes e_{1}, e_{2} \otimes t \otimes t s, e_{2} \otimes t \otimes e_{1}$ and $s t \otimes t \otimes t s$ with respective coefficients $\left(\alpha_{1}^{\prime}\right)^{2}-$ $\phi_{1} \gamma,-\left(\left(\alpha_{3}\right)^{2}-\phi_{1} \gamma\right),\left(\alpha_{1}^{\prime}-\alpha_{3}\right) \gamma$ and $\left(\alpha_{1}^{\prime}-\alpha_{3}\right) \phi_{1}$. Comparing with Eq. C.2b, we get Eq. C.5b.

The method is exactly the same in the case $l=0$ assuming that Eq. C.2c holds.

We get by combining Lemmas C. 1 and C. 2 that if $\lambda=l=0$ as well as $\alpha_{1}^{\prime} \neq \alpha_{3}$, we are in the case 1.a) of Proposition 4.4. If $\alpha_{1}^{\prime}=\alpha_{3}$ instead, we are in the case 1.b).

We now assume that at least one of the two constants $\lambda, l$ is nonzero. Hence, if the double bracket (C.1a)-(C.1b) satisfies (C.2a), it must be such that

$$
\{t, s\}=\alpha_{3}\left(e_{2} \otimes t s-s t \otimes e_{1}\right)+\phi_{0}\left(s t s t \otimes e_{1}-e_{2} \otimes t s t s\right), \quad \alpha_{3}, \phi_{0} \in \mathbb{k},
$$

using Lemma C.1.

Lemma C.3 If Eq. C. $2 b$ holds, then $\phi_{0}=0, l \lambda=0$ and $\alpha_{3}^{2}=\frac{1}{4}$. Moreover, the same statement holds if Eq. C.2c holds.

Proof Developing $\{t, t, s\}$ with Eq. 1.4, we get that the term $e_{2} \otimes t s t s t \otimes t s$ only appears with a factor $\phi_{0}^{2}$. (This is also true for $e_{2} \otimes t \otimes t s t s t s, s t \otimes t s t s t \otimes e_{1}$ and ststst $\otimes t \otimes e_{1}$ with factor $-\phi_{0}^{2}$.) Therefore $\phi_{0}=0$. Under this condition, we obtain that

$$
\{t, t, s\}=\alpha_{3}^{2}\left(s t \otimes t \otimes e_{1}-e_{2} \otimes t \otimes t s\right)+\lambda l(s t \otimes t \otimes t s t s-s t s t \otimes t \otimes t s),
$$

and we get the remaining two equalities by comparing this expression with Eq. C.2b. The computation for $\{s, s, t\}$ with Eq. C.2c is similar and gives the second result.

As a consequence of this lemma, $\phi_{0}$ vanishes and $\alpha_{3}= \pm \frac{1}{2}$ in Eq. C.6. Furthermore, either we have $\lambda \neq 0$ with $l=0$, or we have $l \neq 0$ with $\lambda=0$. These are respectively Case 2 and Case 3 from Proposition 4.4.

\section{Appendix D: Proof of Proposition 4.8}

\section{D.1 Coefficients verifying the triple brackets identities}

The strategy of the proof is given after Proposition 4.8. In this subsection, we gather a list of equalities that the coefficients appearing in the double bracket must satisfy in order for the corresponding triple bracket to satisfy (4.18) or (4.19).

\section{D.1.1 First conditions}

Lemma D.1 If a double bracket given by Eqs. 4.9a-4.9b and 4.10 satisfies (4.18), then we have $\beta_{0}=\beta_{0}^{\prime}=\alpha_{1}=\alpha_{3}^{\prime}=0$. 
Proof Without computing all terms, we can remark that in $\{t, t, s\}$ (obtained from Eq. 1.4 using Eqs. 4.9a, 4.10) the element $s^{3} \otimes 1 \otimes 1$ appears with coefficient $\beta_{0}^{2}$, and so do respectively $1 \otimes 1 \otimes s^{3}, t^{2} s \otimes 1 \otimes 1$ and $1 \otimes 1 \otimes s t^{2}$ with coefficients $-\left(\beta_{0}^{\prime}\right)^{2}, \alpha_{1}^{2}$ and $-\left(\alpha_{3}^{\prime}\right)^{2}$. None of these expressions appear Eq.4.18.

We can go through a similar argument using $\{s, s, t\}$ instead.

Lemma D.2 If a double bracket given by Eqs. $4.9 a-4.9 b$ and 4.10 satisfies (4.19), then we have $\alpha_{0}=\alpha_{0}^{\prime}=\alpha_{1}=\alpha_{3}^{\prime}=0$.

Hence, we are left to discuss the coefficients of the double bracket given by Eqs. 4.9a$4.9 \mathrm{~b}$ and

$$
\begin{aligned}
\{t, s\}= & \gamma_{0} t \otimes t+\gamma_{1} s \otimes s+\alpha_{1}^{\prime} s t \otimes 1+\alpha_{2} t \otimes s+\alpha_{2}^{\prime} s \otimes t+\alpha_{3} 1 \otimes t s \\
& +\beta_{1} t \otimes 1+\beta_{1}^{\prime} 1 \otimes t+\beta_{2} s \otimes 1+\beta_{2}^{\prime} 1 \otimes s+\gamma 1 \otimes 1 .
\end{aligned}
$$

\section{D.1.2 Identities verified by the coefficients when Eq. 4.18 is satisfied}

Lemma D.3 Consider a double bracket defined on A by Eqs. 4.9a, 4.9b and D.1, with $v=0, \lambda \in \mathbb{k}$ and $\mu \in\left\{ \pm \frac{1}{2}\right\}$. Then Eq. 4.18 is satisfied if and only if the following list of identities hold:

$$
\begin{aligned}
& \alpha_{1}^{\prime}, \alpha_{3}= \pm \frac{1}{2}, \quad \alpha_{2}^{2}=\frac{1}{4}+\gamma_{1} \gamma_{0}, \quad\left(\alpha_{2}^{\prime}\right)^{2}=\frac{1}{4}+\gamma_{1} \gamma_{0}, \\
& \frac{1}{4}+\alpha_{2} \alpha_{3}=-\mu\left(\alpha_{2}+\alpha_{3}\right), \quad \frac{1}{4}+\alpha_{1}^{\prime} \alpha_{2}^{\prime}=\mu\left(\alpha_{1}^{\prime}+\alpha_{2}^{\prime}\right), \\
& \gamma_{1}\left(\alpha_{1}^{\prime}-\mu\right)=0, \quad \gamma_{1}\left(\alpha_{2}^{\prime}-\alpha_{2}\right)=0, \quad \gamma_{1}\left(\alpha_{3}+\mu\right)=0, \\
& \beta_{2}\left(\alpha_{1}^{\prime}-\mu\right)=0, \quad \beta_{2}\left(\alpha_{2}^{\prime}-\mu\right)-\gamma_{1} \beta_{1}^{\prime}=0, \quad\left(\beta_{2}-\lambda\right)\left(\alpha_{1}^{\prime}+\alpha_{2}^{\prime}\right)-\gamma_{1} \beta_{1}=0, \\
& \beta_{2}^{\prime}\left(\alpha_{3}+\mu\right)=0, \quad \beta_{2}^{\prime}\left(\alpha_{2}+\mu\right)-\gamma_{1} \beta_{1}=0, \quad\left(\beta_{2}^{\prime}+\lambda\right)\left(\alpha_{2}+\alpha_{3}\right)-\gamma_{1} \beta_{1}^{\prime}=0, \\
& \gamma_{0}\left(\alpha_{1}^{\prime}-\mu\right)=0, \quad \gamma_{0}\left(\alpha_{2}^{\prime}-\alpha_{2}\right)=0, \quad \gamma_{0}\left(\alpha_{3}+\mu\right)=0, \\
& \beta_{1}\left(\alpha_{1}^{\prime}-\alpha_{2}\right)+\gamma_{0}\left(\beta_{2}-\lambda\right)=0, \quad \beta_{1}^{\prime}\left(\alpha_{2}^{\prime}-\alpha_{3}\right)-\gamma_{0}\left(\beta_{2}^{\prime}+\lambda\right)=0, \\
& \beta_{1}^{\prime}\left(\alpha_{1}^{\prime}-\mu\right)-\beta_{1}\left(\alpha_{3}+\mu\right)=0, \quad \beta_{1}\left(\alpha_{2}^{\prime}-\mu\right)-\beta_{1}^{\prime}\left(\alpha_{2}+\mu\right)+\gamma_{0} \lambda=0, \\
& \gamma\left(\alpha_{2}+\mu\right)-\beta_{2} \beta_{1}=0, \quad \gamma\left(\alpha_{1}^{\prime}-\alpha_{3}\right)+\beta_{1}^{\prime}\left(\beta_{2}-\lambda\right)-\beta_{1}\left(\beta_{2}^{\prime}+\lambda\right)=0, \quad \gamma\left(\alpha_{2}^{\prime}-\mu\right)-\beta_{2}^{\prime} \beta_{1}^{\prime}=0, \\
& \beta_{2}^{\prime}\left(\beta_{2}^{\prime}+\lambda\right)-\gamma_{1} \gamma=0, \quad \beta_{2}\left(\beta_{2}-\lambda\right)-\gamma_{1} \gamma=0, \\
& \left(\beta_{2}-\beta_{2}^{\prime}-\lambda\right) \gamma_{1}=0, \quad\left(\beta_{2}-\beta_{2}^{\prime}-\lambda\right) \gamma=0 .
\end{aligned}
$$

Proof We collect now all nonzero terms that appear in the expansion of $\{t, t, s\}$ obtained from Eq. 1.4, leaving the cumbersome (but elementary) computations to the reader.

The coefficients for $t \otimes t \otimes s, s \otimes t \otimes t, 1 \otimes t \otimes t s$ and $s t \otimes t \otimes 1$ are respectively $\gamma_{0} \gamma_{1}-\alpha_{2}^{2},\left(\alpha_{2}^{\prime}\right)^{2}-\gamma_{0} \gamma_{1},-\alpha_{3}^{2}$ and $\left(\alpha_{1}^{\prime}\right)^{2}$. The coefficient for $t \otimes 1 \otimes t s$ and $1 \otimes t^{2} \otimes s$ is $-\alpha_{2} \alpha_{3}-\mu\left(\alpha_{2}+\alpha_{3}\right)$, while we have for $s t \otimes 1 \otimes t$ and $s \otimes t^{2} \otimes 1$ the coefficient $\alpha_{1}^{\prime} \alpha_{2}^{\prime}-\mu\left(\alpha_{1}^{\prime}+\alpha_{2}^{\prime}\right)$. Since these terms appear in Eq. 4.18 , this gives Eqs. D.2a and D.2b. In particular, all the other coefficients in the expansion of $\{t, t, s\}$ must vanish.

The coefficients for $s t \otimes 1 \otimes s, s \otimes t \otimes s$ and $s \otimes 1 \otimes t s$ are respectively $\gamma_{1}\left(\alpha_{1}^{\prime}-\mu\right)$, $\gamma_{1}\left(\alpha_{2}^{\prime}-\alpha_{2}\right)$ and $\gamma_{1}\left(\alpha_{3}+\mu\right)$, which yields Eq. D.2c.

The vanishing of the coefficients for $s t \otimes 1 \otimes 1, s \otimes 1 \otimes t$ and $s \otimes t \otimes 1$ gives successively the three identities in Eq. D.2d. Similarly $1 \otimes 1 \otimes t s, t \otimes 1 \otimes s$ and $1 \otimes t \otimes s$ imply Eq. D.2e, while $1 \otimes t^{2} \otimes t, t \otimes t^{2} \otimes 1$ and $t \otimes t \otimes t$ give Eq. D.2f. 
The coefficients for $t \otimes t \otimes 1,1 \otimes t \otimes t$ and $1 \otimes t^{2} \otimes 1, t \otimes 1 \otimes t$ give Eqs. D.2g and D.2h respectively. With $t \otimes 1 \otimes 1,1 \otimes t \otimes 1$ and $1 \otimes 1 \otimes t$ we obtain (D.2i).

The terms $1 \otimes 1 \otimes s$ and $s \otimes 1 \otimes 1$ give Eq. D.2j. We finally get Eq. D.2k from $s \otimes 1 \otimes s$ and $1 \otimes 1 \otimes 1$.

In the exact same way, we get the next lemma.

Lemma D.4 Consider a double bracket defined on A by Eqs. $4.9 a, 4.9 b$ and D.1, with $v \neq 0$ and $\lambda, \mu \in \mathbb{k}$ satisfying $4\left(\mu^{2}-\lambda v\right)=1$. Then Eq. 4.18 is satisfied if and only if the following list of identities holds :

$$
\begin{aligned}
& \gamma_{0}=0, \quad \gamma_{1}=0, \quad \beta_{2}=0, \quad \beta_{2}^{\prime}=0, \\
& \alpha_{1}^{\prime}, \alpha_{2}, \alpha_{2}^{\prime}, \alpha_{3}= \pm \frac{1}{2}, \quad \frac{1}{4}+\alpha_{2} \alpha_{3}=-\mu\left(\alpha_{2}+\alpha_{3}\right), \quad \frac{1}{4}+\alpha_{1}^{\prime} \alpha_{2}^{\prime}=\mu\left(\alpha_{1}^{\prime}+\alpha_{2}^{\prime}\right), \\
& \alpha_{1}^{\prime}=-\alpha_{2}^{\prime}, \quad \alpha_{2}=-\alpha_{3}, \quad \beta_{1}^{\prime}=-\beta_{1}, \\
& \beta_{1}\left(\alpha_{2}+\alpha_{2}^{\prime}\right)=0, \quad \beta_{1}\left(\alpha_{1}^{\prime}+\alpha_{3}\right)-v \gamma=0, \beta_{1}\left(\alpha_{2}^{\prime}-\alpha_{3}\right)+v \gamma=0, \beta_{1}\left(\alpha_{1}^{\prime}-\alpha_{2}\right)-v \gamma=0, \\
& \gamma\left(\alpha_{2}+\mu\right)=0, \quad \gamma\left(\alpha_{1}^{\prime}-\alpha_{3}\right)=0, \quad \gamma\left(\alpha_{2}^{\prime}-\mu\right)=0, \quad \lambda \gamma=0 .
\end{aligned}
$$

Remark D.5 These results are easily adapted to the case where the double bracket is Poisson, i.e. when the associated triple bracket (1.4) identically vanishes. In such a case, we require $4\left(\mu^{2}-\lambda v\right)=0$ to get $\{t, t, t\}=0$ by [18, Proposition A.1].

If $v=\mu=0$, then $\{t, t, s\}=0$ when the conditions (D.2a)-(D.2k) of Lemma D.3 are satisfied with the extra requirements that all the terms containing a factor $\mu$ are removed, and that all the terms $\pm \frac{1}{2}$ and $\frac{1}{4}$ in Eqs. D.2a-D.2b are removed (in particular $\alpha_{1}^{\prime}=\alpha_{3}=0$ ).

If $v \neq 0$ and $\mu^{2}-\lambda v=0$ then $\{t, t, s\}=0$ when the conditions (D.3a)-(D.3e) of Lemma D. 3 are satisfied with the extra requirements that the terms $\pm \frac{1}{2}$ and $\frac{1}{4}$ appearing in the identities (D.3b) are removed.

\section{D.1.3 Identities verified by the coefficients when Eq. 4.19 is satisfied}

We can obtain the analogues of Lemmae D.3 and D.4 when Eq. 4.19 is satisfied as follows. Using the cyclic antisymmetry of the double bracket, remark that we can get from Eq. D.1

$$
\begin{aligned}
\{s, t\}= & -\gamma_{1} s \otimes s-\gamma_{0} t \otimes t-\alpha_{3} t s \otimes 1-\alpha_{2} s \otimes t-\alpha_{2}^{\prime} t \otimes s-\alpha_{1}^{\prime} 1 \otimes s t \\
& -\beta_{2}^{\prime} s \otimes 1-\beta_{2} 1 \otimes s-\beta_{1}^{\prime} t \otimes 1-\beta_{1} 1 \otimes t-\gamma 1 \otimes 1 .
\end{aligned}
$$

Comparing (4.9a) and (4.9b), then doing the same with Eqs. D.1 and D.4, one can see that to compute $\{s, s, t\}$ one just needs to consider $\{t, t, s\}$ in which we replace all variables $s$ by $t$ and vice-versa, then do the following changes in the coefficients

$$
\begin{aligned}
& \lambda \mapsto l, \quad \mu \mapsto m, \quad v \mapsto n, \\
& \gamma_{0} \mapsto-\gamma_{1}, \quad \gamma_{1} \mapsto-\gamma_{0}, \quad \alpha_{1}^{\prime} \mapsto-\alpha_{3}, \quad \alpha_{2} \mapsto-\alpha_{2}, \quad \alpha_{2}^{\prime} \mapsto-\alpha_{2}^{\prime}, \quad \alpha_{3} \mapsto-\alpha_{1}^{\prime}, \\
& \beta_{1} \mapsto-\beta_{2}^{\prime}, \quad \beta_{1}^{\prime} \mapsto-\beta_{2}, \quad \beta_{2} \mapsto-\beta_{1}^{\prime}, \quad \beta_{2}^{\prime} \mapsto-\beta_{1}, \quad \gamma \mapsto-\gamma .
\end{aligned}
$$

For $n=0, l \in \mathbb{k}$ and $m \in\left\{ \pm \frac{1}{2}\right\}$, we have that Eq. 4.19 is satisfied if and only if the list of identities obtained by applying (D.5) to (D.2a)-(D.2k) is verified.

For $n \neq 0$ and $l, m \in \mathbb{k}$ satisfying $4\left(m^{2}-l n\right)=1$, we have that Eq. 4.19 is satisfied if and only if the list of identities obtained by applying (D.5) to (D.3a)-(D.3e) is verified. 


\section{D.2 Splitting the identities into cases}

Lemma D.6 Consider a reduced double bracket defined on A by Eqs. 4.9a, 4.9b and D.1, with $v=\lambda=0$ and $\mu \in\left\{ \pm \frac{1}{2}\right\}$. Then Eq. 4.18 is satisfied if and only if the double bracket verifies one of the following cases

Case A: For $\gamma_{0}, \gamma_{1} \in \mathbb{k}^{\times}$, then $\gamma \in \mathbb{k}$ is free while

$$
\begin{aligned}
& \alpha_{1}^{\prime}=\mu, \quad \alpha_{3}=-\mu, \quad \alpha_{2}^{\prime}=\alpha_{2} \text { with } \alpha_{2}^{2}=\frac{1}{4}+\gamma_{0} \gamma_{1}, \\
& \beta_{1}=\frac{\gamma_{0} \beta_{2}}{\alpha_{2}-\mu}, \quad \beta_{1}^{\prime}=\frac{\gamma_{0} \beta_{2}}{\alpha_{2}+\mu}, \quad \beta_{2}^{\prime}=\beta_{2} \text { with } \beta_{2}^{2}=\gamma \gamma_{1} .
\end{aligned}
$$

Case B: For $\gamma_{1} \in \mathbb{k}^{\times}, \gamma_{0}=0$, then $\beta_{2} \in \mathbb{k}$ is free while

$$
\alpha_{1}^{\prime}=\mu, \quad \alpha_{3}=-\mu, \quad \beta_{2}^{\prime}=\beta_{2}, \quad \gamma=\frac{\beta_{2}^{2}}{\gamma_{1}},
$$

and one of the following two sets of conditions holds :

$$
\begin{array}{ll}
\text { B1) } \quad \alpha_{2}^{\prime}=\alpha_{2}=\mu, & \beta_{1}^{\prime}=0, \quad \beta_{1}=\frac{2 \mu \beta_{2}}{\gamma_{1}}, \\
\text { B2) } \quad \alpha_{2}^{\prime}=\alpha_{2}=-\mu, \quad \beta_{1}=0, \quad \beta_{1}^{\prime}=-\frac{2 \mu \beta_{2}}{\gamma_{1}} .
\end{array}
$$

Case C: For $\gamma_{0} \in \mathbb{k}^{\times}, \gamma_{1}=0$, then

$$
\alpha_{1}^{\prime}=\mu, \quad \alpha_{3}=-\mu, \quad \beta_{2}^{\prime}=\beta_{2}=0, \quad \gamma=0,
$$

and one of the following two sets of conditions holds :

$$
\begin{gathered}
\text { C1) } \quad \alpha_{2}^{\prime}=\alpha_{2}=\mu, \quad \beta_{1}^{\prime}=0, \quad \beta_{1} \in \mathbb{k}, \\
\text { C2) } \quad \alpha_{2}^{\prime}=\alpha_{2}=-\mu, \quad \beta_{1}=0, \quad \beta_{1} \in \mathbb{k} .
\end{gathered}
$$

Case D: For $\gamma_{0}=\gamma_{1}=0$, then $\beta_{2}^{\prime}=\beta_{2}=0$ and one of the following sets of conditions holds :

$$
\begin{aligned}
& \text { if }\left(\alpha_{1}^{\prime}, \alpha_{3}\right)=(-\mu, \mu) \text {, } \\
& \text { D1) } \quad \alpha_{1}^{\prime}=\alpha_{2}=-\mu, \quad \alpha_{3}=\alpha_{2}^{\prime}=\mu, \quad \beta_{1}=\beta_{1}^{\prime}=\gamma=0 ; \\
& \text { if }\left(\alpha_{1}^{\prime}, \alpha_{3}\right)=(\mu, \mu) \text {, } \\
& \text { D2.1) } \quad \alpha_{1}^{\prime}=\alpha_{2}^{\prime}=\alpha_{3}=\mu, \quad \alpha_{2}=-\mu, \quad \beta_{1}=0, \quad \beta_{1}^{\prime}, \gamma \in \mathbb{k}, \quad(D .12 \mathrm{a}) \\
& \text { D2.2) } \quad \alpha_{1}^{\prime}=\alpha_{3}=\mu, \quad \alpha_{2}=\alpha_{2}^{\prime}=-\mu, \quad \beta_{1}=\beta_{1}^{\prime}=\gamma=0 ; \quad(\mathrm{D} .12 \mathrm{~b}) \\
& \text { if }\left(\alpha_{1}^{\prime}, \alpha_{3}\right)=(-\mu,-\mu) \text {, } \\
& \text { D3.1) } \quad \alpha_{1}^{\prime}=\alpha_{2}=\alpha_{3}=-\mu, \quad \alpha_{2}^{\prime}=\mu, \quad \beta_{1}^{\prime}=0, \quad \beta_{1}, \gamma \in \mathbb{k}, \quad(\mathrm{D} .13 \mathrm{a}) \\
& \text { D3.2) } \quad \alpha_{1}^{\prime}=\alpha_{3}=-\mu, \quad \alpha_{2}=\alpha_{2}^{\prime}=\mu, \quad \beta_{1}=\beta_{1}^{\prime}=\gamma=0 ; \quad \text { (D.13b) } \\
& \text { if }\left(\alpha_{1}^{\prime}, \alpha_{3}\right)=(\mu,-\mu) \text {, } \\
& \text { D4.1) } \quad \alpha_{1}^{\prime}=\alpha_{2}=\alpha_{2}^{\prime}=\mu, \quad \alpha_{3}=-\mu, \quad \beta_{1}^{\prime}=\gamma=0, \quad \beta_{1} \in \mathbb{k}, \quad \text { (D.14a) } \\
& \text { D4.2) } \quad \alpha_{1}^{\prime}=\mu, \quad \alpha_{3}=\alpha_{2}=\alpha_{2}^{\prime}=-\mu, \quad \beta_{1}=\gamma=0, \quad \beta_{1}^{\prime} \in \mathbb{k},(\mathrm{D} .14 \mathrm{~b}) \\
& \text { D4.3) } \alpha_{1}^{\prime}=\alpha_{2}=\mu, \quad \alpha_{3}=\alpha_{2}^{\prime}=-\mu, \quad \gamma=0, \quad \beta_{1}^{\prime}=-\beta_{1}, \beta_{1} \in \mathbb{k}, \quad(\mathrm{D} .14 \mathrm{c}) \\
& \text { D4.4) } \quad \alpha_{1}^{\prime}=\alpha_{2}^{\prime}=\mu, \quad \alpha_{3}=\alpha_{2}=-\mu, \quad \beta_{1}=\beta_{1}^{\prime}=\gamma=0 \text {. (D.14d) }
\end{aligned}
$$

The proof of Lemma D.6 consists in listing the possible coefficients of a reduced double bracket that satisfy Lemma D.3. The next lemma is obtained similarly from Lemma D.4. 
Lemma D.7 Consider a reduced double bracket defined on A by Eqs. 4.9a, 4.9b and D.1, with $v \in \mathbb{k}^{\times}$and $\mu=0, \lambda=\frac{-1}{4 v}$. Then Eq. 4.18 is satisfied if and only if the double bracket verifies

$$
\gamma_{0}=\gamma_{1}=0, \quad \beta_{2}=\beta_{2}^{\prime}=0, \quad \gamma=0, \quad \alpha_{2}= \pm \frac{1}{2},
$$

and one of the following two conditions holds :

$$
\begin{gathered}
\left.A_{v}\right) \quad \alpha_{1}^{\prime}=\alpha_{2}, \quad \alpha_{2}^{\prime}=\alpha_{3}=-\alpha_{2}, \quad \beta_{1}^{\prime}=-\beta_{1}, \quad \beta_{1} \in \mathbb{k}, \\
\left.B_{v}\right) \quad \alpha_{2}^{\prime}=\alpha_{2}, \quad \alpha_{1}^{\prime}=\alpha_{3}=-\alpha_{2}, \quad \beta_{1}=\beta_{1}^{\prime}=0 .
\end{gathered}
$$

Remark D.8 From the discussion in $\S \mathrm{D} .1 .3$, we get that a reduced double bracket defined on $A$ by Eqs. 4.9a, 4.9b and D.1 satisfies (4.19) if and only if the double bracket verifies one of the cases from Lemma D.6 or Lemma D.7 after application of the mapping (D.5) on the different coefficients in each case.

\section{D.3 Finishing the proof}

We need to see which conditions from Lemma D.6 or Lemma D.7 are compatible with at least one of the conditions obtained by applying the mapping (D.5), as explained in Remark D.8.

For example, applying transformation (D.5) to the case D4.4 in Lemma D.6 yields

$$
\begin{aligned}
& \text { Case D4.4 } \gamma_{0}=\gamma_{1}=0, \quad \beta_{1}=\beta_{1}^{\prime}=0, \\
& \alpha_{3}=\alpha_{2}^{\prime}=-m, \quad \alpha_{1}^{\prime}=\alpha_{2}=m, \quad \beta_{2}=\beta_{2}^{\prime}=\gamma=0 .
\end{aligned}
$$

A quick inspection shows that this is compatible with the conditions of the cases D1, D4.3 given by Eqs. D.11, D.14c in Lemma D.6, and $\mathrm{A}_{v}$ given by Eq. D.16a in Lemma D.7. In the first two cases, and under the isomorphism $t \mapsto s, s \mapsto t$ (with $\mu \leftrightarrow m$ ), the obtained double quasi-Poisson brackets satisfy Case 3 of Proposition 4.8 given by Eq. 4.13. In the last case, the double bracket is isomorphic to Case 6 of Proposition 4.8 given by Eq. 4.16 under the same isomorphism (with $m \mapsto \mu, v \mapsto n$ ).

\section{References}

1. Alekseev, A., Kawazumi, N., Kuno, Y., Naef, F.: The Goldman-Turaev Lie bialgebra in genus zero and the Kashiwara-Vergne problem. Adv. Math. 326, 1-53 (2018). arXiv:1703.05813

2. Alekseev, A., Kawazumi, N., Kuno, Y., Naef, F.: The Goldman-Turaev Lie bialgebras and the KashiwaraVergne problem in higher genera. Preprint; arXiv:1804.09566

3. Alekseev, A., Kosmann-Schwarzbach, Y., Meinrenken, E.: Quasi-Poisson manifolds. Canad. J. Math. 54(1), 3-29 (2002). arXiv:math/0006168

4. Bielawski, R.: Quivers and Poisson structures. Manuscripta Math. 141(1-2), 29-49 (2013). arXiv:1108.3222

5. Crawley-Boevey, W.: Preprojective algebras, differential operators and a Conze embedding for deformations of Kleinian singularities. Comment. Math. Helv. 74(4), 548-574 (1999)

6. Crawley-Boevey, W.: Poisson structures on moduli spaces of representations. J. Algebra 325, 205-215 (2011)

7. Crawley-Boevey, W., Shaw, P.: Multiplicative preprojective algebras, middle convolution and the Deligne-Simpson problem. Adv. Math. 201(1), 180-208 (2006). arXiv:math/0404186

8. Chalykh, O., Fairon, M.: Multiplicative quiver varieties and generalised Ruijsenaars-Schneider models. J. Geom. Phys. 121, 413-437 (2017). arXiv:1704.05814

9. Cuntz, J., Quillen, D.: Algebra extensions and nonsingularity. J. Amer. Math. Soc. 8(2), 251-289 (1995)

10. Iyudu, N., Kontsevich, M.: Pre-Calabi-Yau algebras as noncommutative Poisson structures; IHES/m/18/04 (2018) 
11. Kontsevich, M.: Formal (Non)-Commutative symplectic geometry. In: Gelfand, I.M., Corwin, L., Lepowsky, J. (eds.) The Gelfand Mathematical Seminars, 1990-1992, pp. 173-187. Birkhauser, Boston (1993)

12. Kontsevich, M., Rosenberg, A.L.: Noncommutative smooth spaces. The Gelfand Mathematical Seminars, 1996-1999, Gelfand Math. Sem., pp. 85-108. Birkhäuser, Boston (2000)

13. Le Bruyn, L., Procesi, C.: Semisimple representations of quivers. Trans. Amer. Math. Soc. 317(2), 585598 (1990)

14. Massuyeau, G., Turaev, V.: Quasi-Poisson structures on representation spaces of surfaces. Int. Math. Res. Not. IMRN, no. 1, 1-64 (2014). arXiv:1205.4898

15. Odesskii, A.V., Rubtsov, V.N., Sokolov, V.V.: Double Poisson brackets on free associative algebras. Noncommutative birational geometry, representations and combinatorics, Contemp. Math, vol. 592, pp. 225-239. Amer. Math. Soc., Providence (2013). arXiv:1208.2935

16. Odesskii, A.V., Rubtsov, V.N., Sokolov, V.V.: Parameter-dependent associative Yang-Baxter equations and Poisson brackets. Int. J. Geom. Methods Mod. Phys. 11(9), 1460036 (2014). 18 pages, arXiv:1311.4321

17. Pichereau, A., Van de Weyer, G.: Double Poisson cohomology of path algebras of quivers. J. Algebra 319(5), 2166-2208 (2008). arXiv:math/0701837

18. Powell, G.: On double Poisson structures on commutative algebras. J. Geom. Phys. 110, 1-8 (2016). arXiv: 1603.07553

19. Sokolov, V.V.: Classification of constant solutions of the associative Yang-Baxter equation on Mat 3 . Theoret. and Math. Phys. 176(3), 1156-1162 (2013). Russian version appears in Teoret. Mat. Fiz. 176, no. 3, 385-392 (2013). arXiv:1212.6421

20. Van den Bergh, M.: Double Poisson algebras. Trans. Amer. Math. Soc. 360(11), 5711-5769 (2008). arXiv:math/0410528

21. Van den Bergh, M.: Non-commutative quasi-Hamiltonian spaces. In: Poisson geometry in mathematics and physics, vol. 450 of Contemp. Math., pp. 273-299. Amer. Math. Soc., Providence (2008). arXiv:math/0703293

22. Van de Weyer, G.: Double Poisson structures on finite dimensional semi-simple algebras. Algebr. Represent. Theory 11(5), 437-460 (2008)

Publisher's Note Springer Nature remains neutral with regard to jurisdictional claims in published maps and institutional affiliations. 